\title{
THE STRUCTURE OF MACKEY FUNCTORS
}

\author{
JACQUES THEVENAZ AND PETER WEBB
}

\begin{abstract}
Mackey functors are a framework having the common properties of many natural constructions for finite groups, such as group cohomology, representation rings, the Burnside ring, the topological K-theory of classifying spaces, the algebraic K-theory of group rings, the Witt rings of Galois extensions, etc. In this work we first show that the Mackey functors for a group may be identified with the modules for a certain algebra, called the Mackey algebra. The study of Mackey functors is thus the same thing as the study of the representation theory of this algebra. We develop the properties of Mackey functors in the spirit of representation theory, and it emerges that there are great similarities with the representation theory of finite groups. In previous work we had classified the simple Mackey functors and demonstrated semisimplicity in characteristic zero. Here we consider the projective Mackey functors (in arbitrary characteristic), describing many of their features. We show, for example, that the Cartan matrix of the Mackey algebra may be computed from a decomposition matrix in the same way as for group representations. We determine the vertices, sources and Green correspondents of the projective and simple Mackey functors, as well as providing a way to compute the Ext groups for the simple Mackey functors. We parametrize the blocks of Mackey functors and determine the groups for which the Mackey algebra has finite representation type. It turns out that these Mackey algebras are direct sums of simple algebras and Brauer tree algebras. Throughout this theory there is a close connection between the properties of the Mackey functors, and the representations of the group on which they are defined, and of its subgroups. The relationships between these representations are exactly the information encoded by Mackey functors. This observation suggests the use of Mackey functors in a new way, as tools in group representation theory.
\end{abstract}

INDEX OF NOTATION

restriction of Mackey functors, $G$-sets, modules induction of Mackey functors, $G$-sets, modules left adjoint of inflation right adjoint of inflation

$\langle E\rangle$ $M(H) / \sum_{J<H} I_{J}^{H} M(J)$ $N_{G}(H) / H$ subfunctor generated by $E$

Received by the editors April 27, 1993.

1991 Mathematics Subject Classification. Primary 20C20; Secondary 19A22, 20J05, 16G20.

Key words and phrases. Mackey functor, group cohomology, Burnside ring, group representation, block, Brauer tree.

The first author was supported in part by the Swiss NSF, and the second author in part by the NSF. 


$\begin{array}{ll}M^{*} & \text { dual Mackey functor } \\ \mu_{R}(G) & \text { Mackey algebra } \\ \psi & \text { map between Grothendieck groups } \\ \Psi & \text { matrix of } \psi \\ \omega(G) & \text { intermediate category of definition for Mackey functors } \\ \Omega_{R}(G) & \text { category of definition for Mackey functors } \\ B(G) & \text { Burnside ring } \\ B^{G} & \text { Burnside ring Mackey functor } \\ \mathrm{Br}_{P} & \text { Brauer morphism } \\ c_{g} & \text { conjugation } \\ \mathrm{Comack}_{R}(G) & \text { category of cohomological Mackey functors } \\ d_{S T} & \text { decomposition map } \\ e_{H} & \text { Burnside ring idempotents } \\ f_{J} & \text { Burnside ring idempotents } \\ F P_{V} & \text { fixed point Mackey functor } \\ F Q_{V} & \text { fixed quotient Mackey functor } \\ \mathscr{H}_{G} & \text { Hecke category } \\ \operatorname{Mack}_{R}(G) & \text { category of Mackey functors over } R \\ \mathrm{Mack}_{R}(G, J) & \text { category of Mackey functors for which } f_{J} M=M \\ I_{K}^{H} & \text { covariant Mackey functor operation } \\ \mathrm{Inf}_{Q}^{G} & \text { inflation of Mackey functors } \\ P_{H}, V & \text { indecomposable projective Mackey functor } \\ R & \text { commutative ground ring } \\ R_{K}^{H} & \text { contravariant Mackey functor operation } \\ S_{H}, V & \text { simple Mackey functor } \\ T(H, K) & \text { carrier of } H \text { into } K \\ T_{\text {Mack }} & \text { Brauer tree of Mackey functors } \\ T_{\text {Mod }} & \text { Brauer tree of modules } \\ v \mathrm{v}(M) & \text { vertex of } M\end{array}$

\section{INTRODUCTION}

We study the structure of Mackey functors for a finite group $G$ over a commutative ring $R$. We approach this in the spirit of representation theory of algebras and groups and our study here centres mainly around the projective Mackey functors. These are defined to be the projective objects in the category $\operatorname{Mack}_{R}(G)$ of Mackey functors over $R$. In a certain sense they encapsulate all information about Mackey functors since arbitrary Mackey functors may be constructed from them. In considering their properties we introduce the Mackey algebra $\mu_{R}(G)$. This is an algebra of finite rank over $R$ with the property that $\operatorname{Mack}_{R}(G)$ is equivalent to the category of $\mu_{R}(G)$-modules, so that in case $R$ is a field, Mackey functors are the same thing as representations of a certain finite dimensional algebra. This immediately gives us the existence of projective covers, and allows us to talk about idempotents and so forth.

In [20] we classified the simple Mackey functors, showing that they are parameterised by pairs $(H, V)$ where $H$ is a subgroup of $G$ taken up to conjugacy and $V$ is an irreducible $R\left[N_{G}(H) / H\right]$-module. The simple Mackey functor corresponding to this pair is denoted $S_{H, V}$. We immediately obtain a parameterisation of the indecomposable projective Mackey functors in case $R$ is a field, 
since they biject with the simples, and we write $P_{H, V}$ for the projective cover of $S_{H, V}$. Ideally our goal would be a description of the subfunctor lattices of the $P_{H, V}$. Usually this degree of information is too much to ask for, in just the same way as it is only possible in small cases for projective modules over a group algebra. The structure of the Mackey functors is in fact more complicated than with group algebras and the expectation of giving the subfunctor lattices becomes limited by the possibility of describing them in any reasonable way. We sidestep this complication by describing more general properties. There is a decomposition theory similar to that for group representations which shows that the Cartan matrix for Mackey functors is symmetric and non-singular. There are certain fundamental Mackey functors $B^{K} \uparrow_{K}^{G}$ which are induced from the Burnside functor of a subgroup $K$. These are projective, although usually not indecomposable, but we can say exactly when $P_{H, V}$ is a summand of one of these, and with what multiplicity. Arising from this there is a connection with permutation representations and Hecke algebras for $G$, since it emerges that $B^{K} \uparrow_{K}^{G}$ has an endomorphism ring which has as a homomorphic image the Hecke algebra $\operatorname{End}_{R G}\left(R \uparrow_{K}^{G}\right)$, and the kernel is nilpotent.

Mackey functors fall into blocks. As usual the word 'block' has multiple meaning, and we take it to mean either a two-sided direct summand of the Mackey algebra, or the representations of that summand. We describe the division of the simple Mackey functors $S_{H, V}$ into blocks, using some general results giving information about the existence of non-trivial extensions of simple Mackey functors. The result is that the blocks of Mackey functors biject with the ordinary blocks of $G$ together with the blocks of certain sections of $G$. It may seem that Mackey functors, being defined on all subgroups, are rather ill-equipped to deal with blocks, but our result shows that this is not the case.

We consider the question of when the Mackey algebra has finite representation type (that is there are only finitely many isomorphism classes of indecomposable Mackey functors), or when the Mackey algebra is self-injective (that is projective and injective objects coincide). The result is the same for both questions: when $R$ is a field of characteristic $p$, we have finite representation type or self-injectivity if and only if $G$ has a Sylow $p$-subgroup of order (at most) $p$. In this situation, we show that the blocks of the Mackey algebra are either matrix algebras or Brauer tree algebras, and that the Brauer trees are closely related to the ones occuring in the representation theory of the group algebra.

Much of this work was done when we were visiting the ETH Zürich in summer 1989 and we wish to thank the Forschungsinstitut für Mathematik for its hospitality. We also wish to thank Dave Benson for helpful suggestions concerning Brauer trees and Markus Linckelmann for providing a positive answer to a question about higher decomposition numbers.

\section{Preliminaries}

We recall the various equivalent definitions of a Mackey functor which we will find useful. We work with Mackey functors over a commutative ring $R$. The most elementary definition, which is due to Green [11], is that a Mackey functor over $R$ is a mapping

$$
M:\{\text { subgroups of } G\} \rightarrow R \text {-mod }
$$


with morphisms

$$
\begin{aligned}
I_{K}^{H}: M(K) & \rightarrow M(H), \\
R_{K}^{H}: M(H) & \rightarrow M(K), \\
c_{g}: M(H) & \rightarrow M\left({ }^{8} H\right)
\end{aligned}
$$

whenever $K \leq H$ and $g \in G$, such that

(0) $I_{H}^{H}, R_{H}^{H}, c_{h}: M(H) \rightarrow M(H)$ are the identity morphisms for all subgroups $H$ and $h \in H$,

(1) $R_{J}^{K} R_{K}^{H}=R_{J}^{H}$

(2) $\left.I_{K}^{H} I_{J}^{K}=I_{J}^{H}\right\}$ for all subgroups $J \leq K \leq H$,

(3) $c_{g} c_{h}=c_{g h}$ for all $g, h \in G$,

$\left.\begin{array}{l}\text { (4) } R_{g_{K}}^{g_{H}} c_{g}=c_{g} R_{K}^{H} \\ \text { (5) } I_{g K}^{g_{H}} c_{g}=c_{g} I_{K}^{H}\end{array}\right\}$ for all subgroups $K \leq H$ and $g \in G$,

(6) $R_{J}^{H} I_{K}^{H}=\sum_{x \in[J \backslash H / K]} I_{J \cap{ }^{x} K}^{J} c_{x} R_{J^{x} \cap K}^{K}$ for all subgroups $J, K \leq H$.

The morphism $R_{K}^{H}$ is called restriction, $c_{g}$ is called conjugation, while $I_{K}^{H}$ is called induction or sometimes also transfer. Axiom (6) is called the Mackey axiom and explains the terminology.

The definition given by Dress [9] is equivalent to this, provided we restrict to having the category of finite $G$-sets as the domain of definition. We denote by $G$-set the category whose objects are the finite $G$-sets, and where the morphisms are the $G$-equivariant maps. We may define a Mackey functor to be a bifunctor

$$
M=\left(M_{*}, M^{*}\right): G \text {-set } \rightarrow R \text {-mod }
$$

satisfying two conditions. Here a bifunctor means a pair consisting of a covariant functor $M_{*}$ and a contravariant functor $M^{*}$ which agree on objects; thus $M_{*}(X)=M^{*}(X)$ for every $G$-set $X$. We write $M(X)$ for this common value. The two conditions $M$ must satisfy are

(1) for every pullback diagram

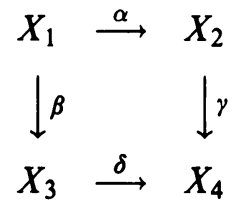

in $G$-set we have $M^{*}(\delta) M_{*}(\gamma)=M_{*}(\beta) M^{*}(\alpha)$.

(2) The two mappings $X \rightarrow X \cup Y \leftarrow Y$ into the disjoint union define an isomorphism $M(X \cup Y) \cong M(X) \oplus M(Y)$ via $M_{*}$ (or equivalently $M^{*}$ ).

The connection between this and the previous definition is that we now write $M(G / H)$ to denote the $R$-module associated to the subgroup $H$, and if $\pi_{H}^{K}$ : $G / H \rightarrow G / K$ denotes the quotient map of coset spaces where $H \leq K$ we have $M_{*}\left(\pi_{H}^{K}\right)=I_{H}^{K}$ and $M^{*}\left(\pi_{H}^{K}\right)=R_{H}^{K}$. The first axiom on pullbacks corresponds to the Mackey axiom in the first definition.

Important examples of Mackey functors are naturally defined on subgroups as in the first definition (e.g., fixed point functors, Burnside functors, etc.). For this reason we shall often use the point of view of the first definition. However many conceptual developments (in particular induction and restriction) are best expressed in the language of the second definition. Thus we shall also use freely 
this second approach. For completeness we mention also a third version of the definition of a Mackey functor which is due to Lindner [15] (see also [25]) and which is probably the most elegant one. Finally a module-theoretic approach will be developed in the next section.

The third definition is based on the following construction. Let $\omega(G)$ be the category whose objects are finite $G$-sets and where the morphisms from $X$ to $Y$ are the equivalence classes of diagrams of $G$-sets $X \leftarrow V \rightarrow Y$. We say that two such diagrams are equivalent if there is a commutative diagram

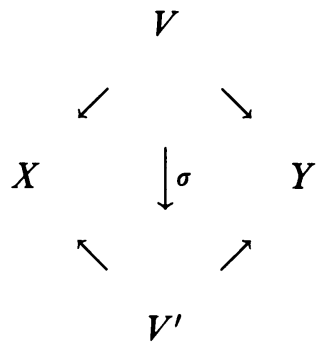

where $\sigma$ is an isomorphism of $G$-sets. To define the composition of morphisms we consider a morphism from $X$ to $Y$ represented by a diagram $X \leftarrow V \rightarrow Y$ and a morphism from $Y$ to $Z$ represented by a diagram $Y \leftarrow W \rightarrow Z$. We form the pullback

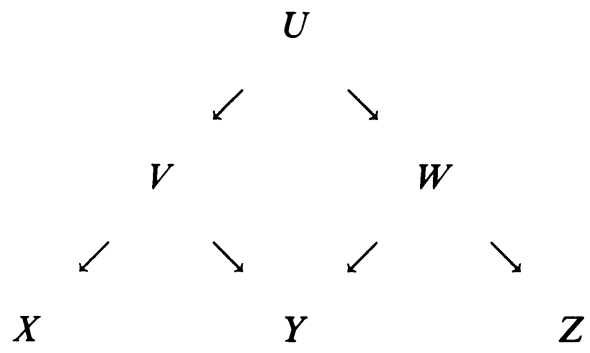

which defines a diagram $X \leftarrow U \rightarrow Z$, hence a morphism from $X$ to $Z$. The set of morphisms $\operatorname{Hom}_{\omega(G)}(X, Y)$ has a structure of monoid using the disjoint union of the intermediate $G$-sets as follows:

$$
(X \stackrel{\alpha}{\leftarrow} V \stackrel{\beta}{\rightarrow} Y)+\left(X \stackrel{\alpha^{\prime}}{\leftarrow} V^{\prime} \stackrel{\beta^{\prime}}{\rightarrow} Y\right)=\left(X \stackrel{\alpha \cup \alpha^{\prime}}{\longleftarrow} V \cup V^{\prime} \stackrel{\beta \cup \beta^{\prime}}{\longrightarrow} Y\right) .
$$

It is clear that the empty set gives rise to a zero element. Moreover it is not difficult to prove that the disjoint union of $G$-sets is both a product and a coproduct in $\omega(G)$, so that $\omega(G)$ is nearly an additive category. In fact, to make it additive, it suffices to turn the monoids $\operatorname{Hom}_{\omega(G)}(X, Y)$ into abelian groups $\mathbb{Z} \operatorname{Hom}_{\omega(G)}(X, Y)$ by the usual universal construction. The situation is particularly easy here because by Proposition 2.2 below, the monoids are free abelian, so that the corresponding abelian groups are free on the same basis. Since we are working over a base ring $R$, we extend scalars to $R$ and define the category $\Omega_{R}(G)$ whose objects are finite $G$-sets and where the set of morphisms from $X$ to $Y$ is the (free) $R$-module $\operatorname{Hom}_{\Omega_{R}(G)}(X, Y)=R \operatorname{Hom}_{\omega(G)}(X, Y)$. Thus $\Omega_{R}(G)$ is an $R$-additive category.

Having defined $\Omega_{R}(G)$, we can give the third definition of a Mackey functor over $R$ : a Mackey functor is simply an $R$-additive functor

$$
M: \Omega_{R}(G) \rightarrow R \text {-mod. }
$$


To recover the second definition of a Mackey functor from this, we note that $M_{*}$ (respectively $M^{*}$ ) is obtained by applying our additive functor to a morphism of the form $X \stackrel{\text { id }}{\leftarrow} X \rightarrow Y$ (respectively $Y \leftarrow X \stackrel{\text { id }}{\rightarrow} X$ ). The requirement in the second definition that a Mackey functor preserves coproducts is replaced here by the condition that the functor is additive. The axiom on pullbacks in the second definition (that is the Mackey axiom in the first one) is incorporated here in the definition of the composition of morphisms in $\omega(G)$. The direct connection with the first definition of a Mackey functor is that again we write $M(G / H)$ to denote the $R$-module associated to the subgroup $H$, and if $H \leq K$ then $M\left(G / K \stackrel{\pi_{H}^{K}}{\leftarrow} G / H \stackrel{1}{\rightarrow} G / H\right)=I_{H}^{K}$ and $M\left(G / H \stackrel{1}{\leftarrow} G / H \stackrel{n_{H}^{K}}{\rightarrow} G / K\right)=R_{H}^{K}$.

We now turn to the proof that $\operatorname{Hom}_{\omega(G)}(X, Y)$ is a free abelian monoid. Since the disjoint union is a product and coproduct in $\omega(G)$, we can decompose both $X$ and $Y$ and it suffices to prove the claim when $X$ and $Y$ are transitive $G$-sets. So we consider morphisms from $G / K$ to $G / H$. Such a morphism will be called basic if it is represented by a diagram of the form $G / K \leftarrow V \rightarrow G / H$, where $V$ is a transitive $G$-set (hence $V \cong G / L$ for some $L$ ).

(2.1) Lemma. Every basic morphism from $G / K$ to $G / H$ in $\omega(G)$ is represented by a diagram

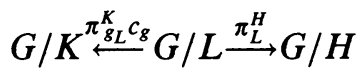

where $L \leq H \cap K^{g}$ and $g \in G$. Such a diagram is equivalent to

$$
G / K \stackrel{\pi_{g_{1}}^{K} c_{L_{1}} c_{g_{1}}}{\longrightarrow} G / L_{1} \stackrel{\pi_{L_{1}}^{H}}{\longrightarrow} G / H
$$

if and only if $K g H=K g_{1} H$ and $L_{1}={ }^{x} L$ for some $x \in H \cap g_{1}^{-1} K g$.

Proof. The commutative squares

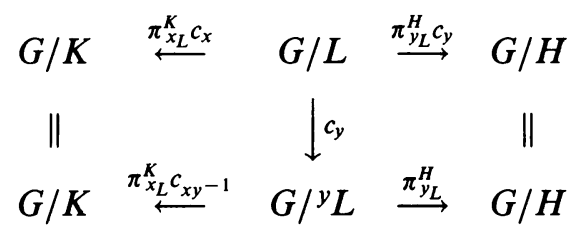

show that the arbitrary diagram at the top is equivalent to one in standard form. Two standard forms are equivalent if and only if we have commutative squares

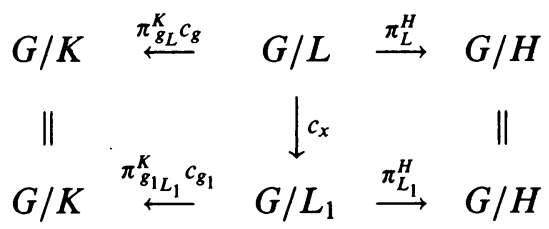

which immediately entails $\pi_{g_{L}}^{K} c_{g}=\pi_{g_{1} L_{1}}^{K} c_{g_{1} x}$ and $\pi_{L}^{H}=\pi_{L_{1}}^{H} c_{x}$ which we rewrite as $c_{g} \pi_{L}^{K^{8}}=c_{g_{1} x} \pi_{L_{1}^{x}}^{K_{1}^{B_{1}}}$ and $\pi_{L}^{H}=c_{x} \pi_{L_{1}^{x}}^{H^{x}}$. We use the property that $c_{a} \pi_{A}^{B}=c_{u} \pi_{U}^{V}$ if and only if $A=U,{ }^{a} B={ }^{u} V$ and $u a^{-1} \in{ }^{a} B$. Thus our conditions are $g_{1} x g^{-1}=k$ for some elements $k \in K$ and $x \in H$ with $L_{1}={ }^{x} L$. Thus $g_{1}=k g x^{-1}$ for some $k \in K$ and $x \in H$, i.e. $K g H=K g_{1} H$, and also $x=g_{1}^{-1} \mathrm{~kg}$ is any element of $H \cap g_{1}^{-1} K g$, which completes the proof. 
(2.2) Proposition. The set of morphisms $\operatorname{Hom}_{\omega(G)}(G / K, G / H)$ is a free abelian monoid, with basis represented by the diagrams

$$
G / K \stackrel{\pi_{g_{L}}^{K} c_{g}}{\longrightarrow} G / L \stackrel{\pi_{L}^{H}}{\longrightarrow} G / H
$$

where $g \in[K \backslash G / H]$ and $L$ is a subgroup of $H \cap K^{g}$ taken up to $H \cap K^{g}$. conjugacy.

Proof. Since any finite $G$-set decomposes uniquely as a disjoint union of transitive $G$-sets (each being isomorphic to some $G / L$ ), any morphism from $G / K$ to $G / H$ is clearly a sum in a unique way of basic morphisms. So the monoid is free. Now for the description of the basis it suffices to settle the question of the equivalence of diagrams representing the same basic morphism. This is clear by the lemma.

To establish our notation we summarise some of the basic constructions on Mackey functors which are used, for example, in [20]. The category of Mackey functors (over $R$ ) for $G$ is denoted $\operatorname{Mack}_{R}(G)$. We denote by

$$
\begin{gathered}
\uparrow_{H}^{G}: \operatorname{Mack}_{R}(H) \rightarrow \operatorname{Mack}_{R}(G), \\
\downarrow_{H}^{G}: \operatorname{Mack}_{R}(G) \rightarrow \operatorname{Mack}_{R}(H),
\end{gathered}
$$

the induction and restriction of Mackey functors. Regarding Mackey functors as defined on $G$-sets, these operations are defined as $M \uparrow_{H}^{G}(X)=M\left(X \downarrow_{H}^{G}\right)$ and $N \downarrow_{H}^{G}(Y)=N\left(Y \uparrow_{H}^{G}\right)$, thus making use of the corresponding notions for $G$-sets. Induction of Mackey functors is both the left and the right adjoint of restriction, a fact which is a little surprising since the same is not true for $G$-sets. Conjugate Mackey functors are defined in a similar fashion: if $M$ is a Mackey functor for a subgroup $H$ of $G$ and $g \in G$, then we have a Mackey functor ${ }^{8} M$ for ${ }^{8} H$ defined by ${ }^{g} M(X)=M\left(g^{-1} X\right)$.

Whenever we have a normal subgroup $N \triangleleft G$ and a Mackey functor $L$ for $Q=G / N$ we can form the inflation $\operatorname{Inf}_{Q}^{G} L$ which is a Mackey functor defined by

$$
\operatorname{Inf}_{Q}^{G} L(K)= \begin{cases}0 & \text { if } K \nsupseteq N, \\ L(K / N) & \text { if } K \supseteq N\end{cases}
$$

with zero restriction and induction morphisms $R_{H}^{K}, I_{H}^{K}$ unless $N \leq H \leq K$, in which case they are the mappings $R_{H / N}^{K / N}, I_{H / N}^{K / N}$ for $L$, and similarly with conjugations. Inflation has a right adjoint and a left adjoint which we describe as follows. Starting with a Mackey functor $M$ for $G$ we obtain Mackey functors $M^{+}$and $M^{-}$on $Q$ by

$$
\begin{aligned}
& M^{+}(K / N)=M(K) / \sum_{\substack{J \leq K \\
J \not \beth^{N}}} I_{J}^{K} M(J), \\
& M^{-}(K / N)=\bigcap_{\substack{J \leq K \\
J \notin 亠 N}} \operatorname{Ker} R_{J}^{K}
\end{aligned}
$$

with induction, restriction and conjugation morphisms inherited from $M$. Then ${ }^{+}$is the left adjoint of $\operatorname{Inf}_{Q}^{G}$ and - is the right adjoint $[20,5.1]$. 
If $M$ is a Mackey functor for $G$ we will write

$$
\bar{M}(H)=M(H) / \sum_{J<H} I_{J}^{H} M(J) .
$$

Note that $\bar{M}(H)$ is an $R \bar{N}_{G}(H)$ module, where we write $\bar{N}_{G}(H)=N_{G}(H) / H$. In particular if $N \triangleleft G$, then $M^{+}(N)=\bar{M}(N)$.

Starting with a module $V$ for $G$ we have the fixed point and fixed quotient functors $F P_{V}$ and $F Q_{V}$, for which $F P_{V}(H)=V^{H}$ and $F Q_{V}(H)=V_{H}$. These satisfy an adjointness property. There is the functor $\operatorname{Mack}_{R}(G) \rightarrow R G$-mod which sends a Mackey functor $M$ to the $R G$-module $M(1)$. Now $F Q$ is left adjoint to this and $F P$ is right adjoint $[20,6.1]$.

A useful property of fixed point functors is the following.

(2.3) Proposition. The map $\operatorname{End}_{\operatorname{Mack}_{R}(G)}\left(F P_{V}\right) \rightarrow \operatorname{End}_{R G}(V)$ given by evaluation at 1 is a ring isomorphism. Thus direct sum decompositions of $F P_{V}$ correspond to direct sum decompositions of $V$. Moreover if $F P_{V}=M_{1} \oplus M_{2}$ then $M_{i}=$ $F P_{W_{i}}$ where $V=W_{1} \oplus W_{2}$.

Proof. The adjunction isomorphism gives

$$
\operatorname{Hom}_{M^{2} k_{R}(G)}\left(F P_{V}, F P_{V}\right) \cong \operatorname{Hom}_{R G}\left(F P_{V}(1), V\right)=\operatorname{End}_{R G}(V) .
$$

If $F P_{V}=M_{1} \oplus M_{2}$ then $V=W_{1} \oplus W_{2}$ as an $R G$-module, where $W_{i}=$ $M_{i}(1)$. Since all restriction maps in a fixed point functor are inclusions, we have $M_{i}(H) \subseteq V^{H} \cap W_{i}=W_{i}^{H}$ and it follows that $M_{i}$ is a subfunctor of $F P_{W_{i}}$. But we have $F P_{W_{1}} \oplus F P_{W_{2}}=F P_{V}=M_{1} \oplus M_{2}$ and this forces the equality $F P_{W_{i}}=M_{i}$.

Using the above tools we construct the simple Mackey functors $S_{H, V}$, which are parametrized by pairs consisting of a subgroup $H$ determined up to conjugacy, and a simple $R \bar{N}_{G}(H)$-module $V$ taken up to isomorphism. First we describe the situation when $H=1$ and $V$ is a simple $R G$-module. It was shown in $[20,7.1]$ that $F P_{V}$ has a unique minimal subfunctor, which is necessarily simple, and this constructs $S_{1, V}$. This subfunctor $S_{1, V}$ is described explicitly by $S_{1, V}(K)=I_{1}^{K}(V) \subseteq V^{K}$, which may also be written as $S_{1, V}(K)=\left(\sum_{g \in K} g\right) \cdot V$. Note in particular that $S_{1, V}(1)=F P_{V}(1)=V$. We occasionally write $S_{H, V}^{G}$ instead of $S_{H, V}$ to emphasize that this is a Mackey functor for $G$. Now if $H$ is any subgroup of $G$ and $V$ is a simple $R \bar{N}_{G}(H)$ module we define $S_{H, V}=\left(\operatorname{Inf}_{\bar{N}(H)}^{N(H)} S_{1, V}^{\bar{N}(H)}\right) \uparrow_{N(H)}^{G}$, and this is in fact a simple Mackey functor. The $S_{H, V}$ so constructed constitute a complete set of representatives for the isomorphism classes of simple Mackey functors [20, 8.3]. Another important property of the simple Mackey functors $S_{H, V}$ which we will use repeatedly is that $S_{H, V}(K)=0$ unless $K \geq_{G} H$, and $S_{H, V}(H)=V$. This is an easy consequence of the description of $S_{H, V}$ given above.

Occasionally we will refer to the Mackey functor generated by a set of elements. This will mean that we have a Mackey functor $M$ and for each subgroup $H \leq G$ there is specified a set of elements $E(H) \subseteq M(H)$. We put

$$
\langle E\rangle=\bigcap\{N \mid N \text { is a subfunctor of } M, E(H) \subseteq N(H) \text { for all subgroups } H\},
$$

and this is the subfunctor generated by $E$. If we do not explicitly specify $E(H)$ for some subgroup $H$ then we take $E(H)=0$. We now quote Proposition 2.1 
of [20], which gives an explicit description of this construction in an important special case.

(2.4) Proposition. Let $M$ be a Mackey functor for $G$. Let $\mathscr{X}$ be a family of subgroups of $G$, closed under conjugation and taking subgroups, and for each $X \in \mathscr{Z}$, let $E(X)$ be an $R$-submodule of $M(X)$ such that

$$
I_{Y}^{X}(E(Y)) \subseteq E(X), \quad R_{Y}^{X}(E(X)) \subseteq E(Y), \quad c_{g}(E(X))=E\left({ }^{g} X\right),
$$

for all $Y \leq X \in \mathscr{X}$ and $g \in G$ (so that $E$ is a "Mackey functor defined on $\mathscr{X} "$ "). Then for each subgroup $H$ of $G$

$$
\langle E\rangle(H)=\sum_{\substack{X \in \mathscr{O} \\ X \leq H}} I_{X}^{H}(E(X)) .
$$

Moreover if $H \in \mathscr{X}$ then $\langle E\rangle(H)=E(H)$.

\section{The MACKey ALgebra}

The category of Mackey functors for $G$ over $R$ is equivalent to the category of modules for an $R$-algebra $\mu_{R}(G)$ of finite rank which we call the Mackey algebra. We define $\mu_{R}(G)$ in this section and develop its elementary properties. It can be convenient to think of Mackey functors in these terms because one may be more familiar with modules than with functors. For example one may be concerned about the existence of projective covers, or of blocks of Mackey functors. The quickest way to deal with these questions is to put them into the standard setting of modules.

We define the Mackey algebra in two ways, the first being intuitively appealing and the second useful in computation. For the first definition we consider the quiver whose vertices are the subgroups of $G$ and where we have edges

$$
H \bullet \stackrel{I_{H}^{K}}{\longrightarrow} \bullet K \quad H \bullet \stackrel{R_{H}^{K}}{\longleftarrow} \bullet K
$$

for each pair of subgroups $H \leq K$, and also an edge

$$
H \bullet \stackrel{c_{g}, H}{\longrightarrow} \bullet{ }^{g} H
$$

for each pair $(g, H)$ with $g \in G, H \leq G$. Because the notation gets very cumbersome otherwise, we write $c_{g}$ instead of $c_{g, H}$ when there is no confusion. We define the Mackey algebra $\mu_{R}(G)$ to be $\Lambda / J$ where $\Lambda$ is the path algebra over $R$ of this quiver and $J$ is the 2-sided ideal of $\Lambda$ generated by the relations (1) - (6) in the definition of a Mackey functor, together with

$\left(0^{\prime}\right)$ For all $H \leq G$ and $h \in H, I_{H}^{H}=R_{H}^{H}=c_{h, H}$ is the path of length zero at $H$.

It is apparent that a Mackey functor in the sense of the first definition is precisely a representation over $R$ of the above quiver with relations, since it consists of the specification of an $R$-module for each vertex of the quiver, with morphisms between these modules for each arrow of the quiver. These morphisms must satisfy exactly the relations we put on the quiver. We thus see that Mackey functors over $R$ may be identified as $\mu_{R}(G)$-modules, the correspondence being that from a Mackey functor $M$ we may construct the $\mu_{R}(G)$ module $\bigoplus_{H \leq G} M(H)$, and conversely given a $\mu_{R}(G)$-module $A$ we recover the Mackey functor $M$ by setting $M(H)=I_{H}^{H} \cdot A$. 
It is clear that $\mu_{R}(G)$ is finitely generated as an $R$-module. We shall see below that it is actually a free $R$-module, a fact which is not obvious from the definition. In case $R$ is a field, $\mu_{R}(G)$ is a finite dimensional $R$-algebra and so we automatically have the existence of projective covers, the Krull-Schmidt theorem and so forth for these Mackey functors.

It is convenient to give another definition of the Mackey algebra using the category $\Omega_{R}(G)$ which was introduced in the third definition of Mackey functors. We define an algebra

$$
\mu_{R}^{\prime}(G)=\bigoplus_{H, K \leq G} \operatorname{Hom}_{\Omega_{R}(G)}(G / H, G / K)
$$

which we will show in 3.3 is isomorphic to $\mu_{R}(G)$. The multiplication in $\mu_{R}^{\prime}(G)$ is defined on components in the direct sum by composition of morphisms in the category $\Omega_{R}(G)$, or zero if two morphisms cannot be composed. After 3.3 we will identify $\mu_{R}^{\prime}(G)$ with $\mu_{R}(G)$, but for the moment we retain the separate notations.

(3.1) Proposition. The category of Mackey functors (i.e. additive functors $\Omega_{R}(G)$ $\rightarrow R-$ mod) is isomorphic to $\mu_{R}^{\prime}(G)$-mod.

Proof. The correspondence is the same as the one between representations of a quiver and modules for its path algebra. Given an additive functor $M$ we may construct the $\mu_{R}^{\prime}(G)$-module $\bigoplus_{H \leq G} M(G / H)$; conversely given a $\mu_{R}^{\prime}(G)$ module $V$ we recover the additive functor $M$ by setting $M(G / H)=\mathrm{id}_{G / H} \cdot V$ and extending the definition of $M$ to an arbitrary $G$-set $X \cong \bigcup_{i} G / H_{i}$ by means of a direct sum formula $M(X)=\bigoplus_{i} M(G / H)$.

It is immediate from Proposition 2.2 that $\mu_{R}^{\prime}(G)$ is free as an $R$-module and is of finite rank, with an explicit basis given by 2.2 :

(3.2) Proposition. The algebra $\mu_{R}^{\prime}(G)$ is free as an $R$-module with basis represented by the elements $G / K \stackrel{\pi_{g_{L}}^{K} c_{g}}{\longleftarrow} G / L \stackrel{\pi_{L}^{H}}{\longrightarrow} G / H$ where $H, K$ are arbitrary subgroups of $G, g$ represents a double coset in $K \backslash G / H$ and $L \leq H \cap K^{g}$ is taken up to $H \cap K^{g}$-conjugacy.

This last result provides a mechanical, if tedious, way to compute the rank of $\mu_{R}^{\prime}(G)$.

(3.3) Proposition. The two algebras $\mu_{R}(G)$ and $\mu_{R}^{\prime}(G)$ are isomorphic.

Proof. Recall that $\mu_{R}(G)=\Lambda / J$ where $\Lambda$ is the path algebra of a quiver and $J$ is an ideal of relations. We define an algebra homomorphism $g: \Lambda / J \rightarrow \mu_{R}^{\prime}(G)$ by defining a homomorphism $\hat{g}: \Lambda \rightarrow \mu_{R}^{\prime}(G)$ which is zero on $J$. The typical basis element of $\Lambda$ can be written as a product of elements $I, c$ and $R$ of the form

$$
I_{g_{n} L_{n}}^{H_{n+1}} c_{g_{n}} R_{L_{n}}^{H_{n}} \cdots I_{g_{1} L_{1}}^{H_{2}} c_{g_{1}} R_{L_{1}}^{H_{1}}
$$

provided we allow the possibility to put symbols $R_{L_{1}}^{L_{1}}, I_{L_{1}}^{L_{1}}$, etc. which represent the identity path at $L_{1}$. Apart from such extra identity paths, the definition of the path algebra says that this expression is unique. We define $\hat{g}: \Lambda \rightarrow \mu_{R}^{\prime}(G)$ 
on these basis elements by putting

$$
\begin{array}{r}
\hat{g}\left(I_{g_{n} L_{n}}^{H_{n+1}} c_{g_{n}} R_{L_{n}}^{H_{n}} \cdots I_{g_{1} L_{1}}^{H_{2}} c_{g_{1}} R_{L_{1}}^{H_{1}}\right)=\left(G / H_{n+1} \stackrel{\pi_{g_{n+1}}^{H_{n+1}} c_{g_{n}}}{\longleftarrow} G / L_{n} \stackrel{\pi_{L_{n}}^{H_{n}}}{\longrightarrow} G / H_{n}\right) \\
\cdots\left(G / H_{2} \stackrel{\pi_{g_{1} L_{1}}^{H_{2}} c_{g_{1}}}{\longleftarrow} G / L_{1} \stackrel{\pi_{L_{1}}^{H_{1}}}{\longrightarrow} G / H_{1}\right) .
\end{array}
$$

The set of paths in the quiver (together with 0 ) is closed under multiplication and the above assignment preserves this structure, so it extends by linearity to an algebra homomorphism $\hat{g}$ defined on all of $\Lambda$. This vanishes on $J$ since the category $\Omega_{R}(G)$ is constructed precisely so that the Mackey functor relations are satisfied there.

We construct now an $R$-linear homomorphism $f: \mu_{R}^{\prime}(G) \rightarrow \Lambda / J$ by defining it on the basis of $\mu_{R}^{\prime}(G)$. We define

$$
f\left(G / K \stackrel{\pi_{g_{L}}^{K} c_{g}}{\longleftarrow} G / L \stackrel{\pi_{L}^{H}}{\longrightarrow} G / H\right)=I_{g L}^{K} c_{g} R_{L}^{H}+J
$$

This definition is independent of the choice of representative of the basis element of this form, since if

$$
G / K \stackrel{\pi_{g_{1 L_{1}}}^{K} c_{g_{1}}}{\longleftarrow} G / L_{1} \stackrel{\pi_{L_{1}}^{H}}{\longrightarrow} G / H
$$

is in the same equivalence class, then, using the notation in Lemma 2.1, its image under $f$ is

$$
\begin{aligned}
I_{g_{1} L_{1}}^{K} c_{g_{1}} R_{L_{1}}^{H}+J & =I_{g_{1} x}^{K} c_{g_{1}} R_{x_{L}}^{H}+J \\
& =c_{k^{-1}} I_{k g_{L}}^{K} c_{g_{1}} R_{x_{L}}^{H} c_{x}+J \\
& =I_{g_{L}}^{K} c_{k^{-1}} c_{g_{1}} c_{x} R_{L}^{H}+J \\
& =I_{g_{L}}^{K} c_{g} R_{L}^{H}+J
\end{aligned}
$$

where $x=g_{1}^{-1} k g$ and $L_{1}={ }^{x} L$, since these transformations are all obtained by applying the Mackey functor relations. It is immediate that $f$ and $g$ are mutually inverse.

We shall from now on identify the two algebras $\mu_{R}(G)$ and $\mu_{R}^{\prime}(G)$. Although it was useful to introduce $\mu_{R}^{\prime}(G)$ to make it clear that the Mackey algebra is free as an $R$-module, it is usually more convenient to write elements of $\mu_{R}(G)$ as sums of products of symbols $I_{K}^{H}, R_{K}^{H}, c_{g, H}$ using the quiver definition of the algebra, rather than as sums of diagrams as in the second definition. With this notation the statement of 3.2 becomes that $\mu_{R}(G)$ is a free $R$-module with a basis over $R$ consisting of the elements

$\left\{I_{8 L}^{K} c_{g} R_{L}^{H} \mid H, K \leq G, g \in[K \backslash G / H], L \leq H \cap K^{g}\right.$ up to $H \cap K^{g}$-conjugacy $\}$.

The fact that $\mu_{R}(G)$ is a free $R$-module is fundamental in our discussion of decomposition theory in Section 7.

(3.4) Proposition. For each subgroup $H \leq G$, the elements $c_{g, H}$ with $g$ ranging over a set of representatives for $\bar{N}_{G}(H)$ span a subalgebra of $\mu_{R}(G)$ isomorphic to the group algebra $R \bar{N}_{G}(H)$.

Proof. These elements certainly multiply together in the manner of the elements of $\bar{N}_{G}(H)$. In particular their span is a subalgebra of $\mu_{R}(G)$ and the only 
question is whether these elements are linearly independent. This is guaranteed by the description of the basis of $\mu_{R}(G)$, of which they form part.

In view of this result we immediately obtain certain idempotents in $\mu_{R}(G)$, namely the group ring idempotents in $R \bar{N}_{G}(H)$ for each subgroup $H$ of $G$. We will see in later sections that primitive idempotents in $R \bar{N}_{G}(H)$ do not remain primitive in $\mu_{R}(G)$, and in fact (by an extension of Theorem 8.6) we can give the exact decomposition into primitive idempotents of $\mu_{R}(G)$. For the moment we can at least say when $R$ is a field that since conjugacy classes of idempotents biject with simple modules there is for each simple $\mu_{R}(G)$-module $S_{H, V}$ a primitive idempotent $e_{H, V}$ determined up to conjugacy by the fact that $e_{H, V} \cdot S_{H, V} \neq 0$. Then $P_{H, V}=\mu_{R}(G) \cdot e_{H, V}$ is the projective cover of $S_{H, V}$.

We have various reasons for introducing the Mackey algebra. One of them is that it allows us to borrow standard constructions and results from module theory without formality, such as the existence of projective covers, tensor products and the fact that the Krull-Schmidt theorem holds. Another is that it provides us with our first example of a projective Mackey functor, namely the regular representation. In fact this might be termed a free Mackey functor, and it satisfies a universal property associated with this usage.

We now interpret a known result in terms of the Mackey algebra.

(3.5) Theorem. Let $k$ be a field in which $|G|$ is invertible. Then $\mu_{k}(G)$ is a semisimple $k$-algebra.

Proof. In this situation every Mackey functor is a direct sum of simple Mackey functors, by one of the main results of [20].

A new proof of this theorem will be given in Section 14.

(3.6) Theorem. Let $k$ be a field. Then $k$ is a splitting field for $\mu_{k}(G)$ if and only if $k$ is a splitting field for the representations of $\bar{N}_{G}(H)$ for every subgroup $H \leq G$.

Proof. We apply Theorem 3.1 of [20] which characterizes simple Mackey functors $S$ by the property that if $H$ is a minimum subgroup of $S$ (that is, $S(H) \neq 0$ and $S(K)=0$ if $K<H)$ then

(1) $\bigcap_{\substack{g \in G \\ g \leq K}} \operatorname{Ker} R_{g_{H}}^{K}=0$ for all subgroups $K \leq G$,

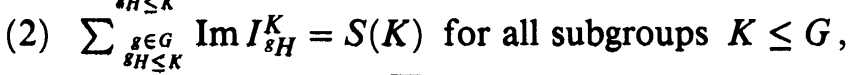

(3) $S(H)$ is a simple $k \bar{N}_{G}(H)$-module.

Conditions (1) and (2) remain intact on extension of scalars, and if $k$ is a splitting field for $\bar{N}_{G}(H)$ then so does (3), so $S$ remains simple. Thus if $k$ is a splitting field for every group $\bar{N}_{G}(H)$, it is a splitting field for $\mu_{k}(G)$.

On the other hand, if $k$ is not a splitting field for some $\bar{N}_{G}(H)$ then there is a simple $k \bar{N}_{G}(H)$-module $V$ and a field extension $k^{\prime} \supseteq k$ so that $k^{\prime} \otimes_{k} V$ is not simple. If $S_{H, V}$ is the corresponding simple Mackey functor then $k^{\prime} \otimes_{k} S_{H, V}$ fails condition (3) of the characterization, so is not simple, and so $k$ is not a splitting field for $\mu_{k}(G)$.

\section{DuAlity For MACKey Functors}

For simplicity in this section we will work throughout with a field $k$ as our base ring. We define the dual of a Mackey functor $M$ to be the Mackey 
functor $M^{*}$, where $M^{*}(H)$ is the $k$-module dual $D M(H)=\operatorname{Hom}_{k}(M(H), k)$ for each subgroup $H$, and with

$$
I_{H}^{K}=D\left(R_{H}^{K}\right), \quad R_{H}^{K}=D\left(I_{H}^{K}\right), \quad c_{g}=D\left(c_{g^{-1}}\right)
$$

where on the right-hand side we have the mappings induced on the dual spaces by the restriction, induction and conjugation for $M$. Thus as far as the conjugations are concerned, $M^{*}(H)$ is the contragredient representation of the group $\bar{N}_{G}(H)$.

It is convenient to interpret duality in terms of the Mackey algebra. Mackey functors may naturally be regarded as left $\mu_{k}(G)$-modules. One readily sees that right $\mu_{k}(G)$-modules may be regarded in the same way as what one might call 'contravariant Mackey functors'. In terms of the third Mackey functor definition we mean by this a contravariant additive functor $\Omega_{k}(G) \rightarrow k$-mod. There are two ways in which starting with a left $\mu_{k}(G)$-module we can obtain a right $\mu_{k}(G)$-module and vice versa. The first is by taking $k$-module duals. If $M$ is a left $\mu_{k}(G)$-module then

$$
D M=\operatorname{Hom}_{k}(M, k)
$$

acquires the structure of a right $\mu_{k}(G)$-module in the usual way via

$$
(f x)(m)=f(x m), \quad f \in D M, \quad x \in \mu_{k}(G), \quad m \in M .
$$

The second comes from the observation that $\mu_{k}(G)$ has an antiautomorphism specified by

$$
I_{8 L}^{K} c_{g} R_{L}^{H} \mapsto I_{L}^{H} c_{g^{-1}} R_{g_{L}}^{K} .
$$

Thus $R$ 's and $I$ 's are interchanged, $c_{g}$ is replaced by $c_{g^{-1}}$ and the order of multiplication is reversed. The fact that this gives an antiautomorphism follows either from the fact that $\Omega_{k}(G)$ has a similar antiautomorphism, or else by verifying that the ideal of relations in the path algebra is preserved. In particular, the Mackey decomposition formula is left unchanged. Let us denote this antiautomorphism by $x \mapsto \bar{x}, x \in \mu_{k}(G)$. If $M$ is a left $\mu_{k}(G)$-module we denote by $M^{\text {op }}$ the right $\mu_{k}(G)$-module with the same elements as $M$ and

$$
m x=\bar{x} m, \quad x \in \mu_{k}(G), \quad m \in M .
$$

In a similar way if $M$ is a right $\mu_{k}(G)$-module we obtain left modules $D M$ and $M^{\mathrm{op}}$. It is apparent that ${ }^{\mathrm{op}}$ gives an isomorphism of categories between left and right $\mu_{k}(G)$-modules, and $D$ provides a contravariant duality between these categories. Thus, for example, op preserves the classes of projectives and injectives and $D$ interchanges them.

In these terms we can now define define $M^{*}=D M^{\text {op }}$, and this coincides with the previous definition. It is apparent that it does not matter in which order we apply $D$ and op , so we do not need parentheses. Now ${ }^{*}$ is a duality on Mackey functors whose square is the identity. It preserves exact sequences and interchanges projectives and injectives. This proves parts (i) and (ii) of the next proposition.

(4.1) Proposition. Let $k$ be a field. Then

(i) the Mackey functor $P$ is projective if and only if $P^{*}$ is injective, 
(ii) the composition factors of a dual Mackey functor $M^{*}$ are the duals of the composition factors of $M$,

(iii) for every $k G$-module $V,\left(F P_{V}\right)^{*} \cong F Q_{V^{*}}$, and

(iv) for every subgroup $H$ and $k \bar{N}_{G}(H)$-module $V, S_{H, V}^{*} \cong S_{H, V^{*}}$.

Proof. (iii) The functor $V \mapsto\left(F P_{V^{*}}\right)^{*}$ is left adjoint to evaluation at the identity, as is seen from the following sequence of natural bijections:

$$
\begin{aligned}
\operatorname{Hom}\left(\left(F P_{V^{*}}\right)^{*}, M\right) & \leftrightarrow \operatorname{Hom}\left(M^{*}, F P_{V^{*}}\right) \\
& \leftrightarrow \operatorname{Hom}\left(M^{*}(1), V^{*}\right) \\
& \leftrightarrow \operatorname{Hom}\left(M(1)^{*}, V^{*}\right) \\
& \leftrightarrow \operatorname{Hom}(V, M(1)) .
\end{aligned}
$$

Since we already know that $F Q_{V}$ is the left adjoint, we obtain the desired isomorphism of functors.

(iv) For $H=1$ this follows from the construction of $S_{1, V}$ as the unique simple Mackey functor appearing in an exact sequence $0 \rightarrow S_{1, V} \rightarrow F P_{V}$. Applying * gives an exact sequence $F Q_{V^{*}} \rightarrow S_{1, V}^{*} \rightarrow 0$, but the unique simple quotient of $F Q_{V^{*}}$ is $S_{1, V^{*}}$, so we have the desired isomorphism. For an arbitrary $H$, remember that $S_{H, V}=\left(\operatorname{Inf}_{\bar{N}(H)}^{N(H)} S_{1, V}^{\bar{N}(H)}\right) \uparrow_{N(H)}^{G}$. It is easy to check that taking the dual commutes with both inflation and induction. This reduces to the case of a simple functor $S_{1, V}$ which we have just seen.

(4.2) Lemma. Let $e_{H, V} \in \mu_{k}(G)$ be a primitive idempotent for which $e_{H, V} S_{H, V}$ $\neq 0$. Then $\overline{e_{H, V}}$ is a primitive idempotent for which $\overline{e_{H, V}} S_{H, V^{*}} \neq 0$.

Proof. Evidently $e_{H, V}$ is a primitive idempotent if and only if $\overline{e_{H, V}}$ is a primitive idempotent. On the assumption that $e_{H, V} S_{H, V} \neq 0$ we have

$$
\begin{aligned}
\overline{e_{H, V}} S_{H, V} & =\overline{e_{H, V}} S_{H, V}^{*} \\
& =\overline{e_{H, V}} D\left(S_{H, V}\right)^{\mathrm{op}} \\
& =\left(D S_{H, V}\right) e_{H, V} \\
& =D\left(e_{H, V} S_{H, V}\right) \\
& \neq 0 .
\end{aligned}
$$

In the last equality, one has to interpret a linear form on $S_{H, V}$ lying in $\left(D S_{H, V}\right) e_{H, V}$ as a linear form on $S_{H, V}$ vanishing on $\left(1-e_{H, V}\right) S_{H, V}$.

We step ahead of ourselves for a moment to deduce a corollary which would also find a fitting place when we come to consider the Cartan matrix in Section 7. We use the notation $P_{H, V}$ to denote the projective cover of $S_{H, V}$. Since we are working over a field $k$ and Mackey functors are modules for the finite dimensional $k$-algebra $\mu_{k}(G)$, these projective covers always exist. In fact $P_{H, V}$ corresponds to the $\mu_{k}(G)$-module $\mu_{k}(G) \cdot e_{H, V}$.

(4.3) Corollary. The multiplicity of $S_{H, V}$ as a composition factor of $P_{K, W}$ equals the multiplicity of $S_{K, W^{*}}$ as a composition factor of $P_{H, V^{*}}$.

Proof. The antiautomorphism - induces an isomorphism of vector spaces

$$
e_{H, V} \mu(G) e_{K, W} \cong \overline{e_{K, W}} \mu(G) \overline{e_{H, V}}
$$


which we can write as $e_{K, W^{*}} \mu(G) e_{H, V^{*}}$. These vector spaces thus have the same dimension, which is what we need.

\section{RELATIONS WITH SUBGROUPS AND QUOTIENTS}

There are certain properties of induction and restriction of Mackey functors and also of the fixed point and fixed quotient functors which follow immediately from their adjointness properties. Thus a functor which has both a left and a right adjoint is exact [13, II.7.7], the left adjoint sends projectives to projectives and the right adjoint sends injectives to injectives [13, II.10.2]. We know that if $H \leq G$ then the induction functor $\uparrow_{H}^{G}$ is both the left and right adjoint of the restriction functor $\downarrow_{H}^{G}$. Also the functor (1): $\operatorname{Mack}_{R}(G) \rightarrow R G$-mod which sends a Mackey functor $M$ to the evaluation $M(1)$ has left adjoint $F Q$ and right adjoint $F P$. We summarise the consequences of this situation in the next result, which can also be proved in a direct fashion.

(5.1) Proposition. Let $H \leq G$. Then

(i) induction $\uparrow_{H}^{G}: \operatorname{Mack}_{R}(H) \rightarrow \operatorname{Mack}_{R}(G)$ and restriction $\downarrow_{H}^{G}$ are both exact functors,

(ii) both functors $\uparrow_{H}^{G}$ and $\downarrow_{H}^{G}$ send projectives to projectives and injectives to injectives, and

(iii) if $V$ is a projective $R G$-module then $F Q_{V}$ is a projective Mackey functor; if $V$ is an injective $R G$-module then $F P_{V}$ is an injective Mackey functor.

Induction and restriction of Mackey functors are related to induction and restriction of $R G$-modules in the following way. We use the notation $\uparrow_{H}^{G}$ and $\downarrow_{H}^{G}$ for induction and restriction of modules, as well as of Mackey functors.

(5.2) Proposition. Let $H \leq G$. Let $M$ be a Mackey functor for $G$ and $N a$ Mackey functor for $H$. Let $V$ be an $R G$-module and $W$ an $R H$-module. Then

(i) $N \uparrow_{H}^{G}(1) \cong N(1) \uparrow_{H}^{G}$ and $M \downarrow_{H}^{G}(1) \cong M(1) \downarrow_{H}^{G}$.

(ii) $F P_{W} \uparrow_{H}^{G} \cong F P_{W \uparrow_{H}^{G}}$ and $F P_{V} \downarrow_{H}^{G} \cong F P_{V \downarrow_{H}^{G}}$.

(iii) $F Q_{W} \uparrow_{H}^{G} \cong F Q_{W \uparrow_{H}^{G}}$ and $F Q_{V} \downarrow_{H}^{G} \cong F Q_{V \downarrow_{H}^{G}}$.

Proof. (i) Using $G$-set notation we have

$$
N \uparrow_{H}^{G}(G / 1)=N\left((G / 1) \downarrow_{H}^{G}\right)=N\left(\bigcup_{g \in[G / H]} H / 1\right)=\bigoplus_{g \in[G / H]} N(H / 1) .
$$

One sees that the group $G$ permutes the summands transitively and that the action of $H$ on the first summand comes from the Mackey functor structure of $N$. Thus we obtain the induced module $N(1) \uparrow_{H}^{G}$. The second claim is trivial.

(ii) We have a series of adjunctions

$$
\begin{aligned}
\operatorname{Hom}_{M^{\prime} k_{R}(G)}\left(M, F P_{W} \uparrow_{H}^{G}\right) & \cong \operatorname{Hom}_{M_{a c k}(H)}\left(M \downarrow_{H}^{G}, F P_{W}\right) \\
& \cong \operatorname{Hom}_{R H}\left(M \downarrow_{H}^{G}(1), W\right) \\
& =\operatorname{Hom}_{R H}\left(M(1) \downarrow_{H}^{G}, W\right) \\
& \cong \operatorname{Hom}_{R G}\left(M(1), W \uparrow_{H}^{G}\right) \\
& \cong \operatorname{Hom}_{\operatorname{Mack}_{R}(G)}\left(M, F P_{W \uparrow_{H}^{G}}\right)
\end{aligned}
$$


and the first result follows. The second is proved in a similar fashion.

(iii) The proof is analogous to the proof of (ii), using this time the fact that $F Q$ is left adjoint of the evaluation at 1 .

Another useful result is that the Mackey decomposition formula holds for induction and restriction of Mackey functors.

(5.3) Proposition. Let $H, K \leq G$ and let $M$ be a Mackey functor for $H$. Then

$$
M \uparrow_{H}^{G} \downarrow_{K}^{G} \cong \bigoplus_{g \in[K \backslash G / H]}\left({ }^{g} M\right) \downarrow_{K \cap 8 H}^{s_{H}} \uparrow_{K \cap 8 H}^{K} .
$$

Proof. This is an easy consequence of the corresponding result for $G$-sets. For a $K$-set $X$, we have

$$
\begin{aligned}
& M \uparrow_{H}^{G} \downarrow_{K}^{G}(X)=M\left(X \uparrow_{K}^{G} \downarrow_{H}^{G}\right) \cong M\left(\bigcup_{g \in[K \backslash G / H]}\left(g^{-1} X\right) \downarrow_{g^{-1} K \cap H^{-1} K \uparrow_{g^{-1} K \cap H}^{H}}^{g^{-1}}\right) \\
& =\bigoplus_{g \in[K \backslash G / H]} M\left(\left(g^{-1} X\right) \downarrow_{g^{-1} K \cap H^{-1}}^{\uparrow^{H}}{ }_{g^{-1} K \cap H}\right)
\end{aligned}
$$

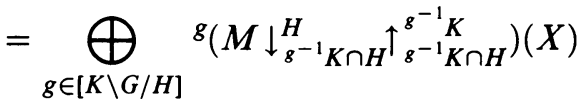

$$
\begin{aligned}
& =\bigoplus_{g \in[K \backslash G / H]}\left({ }^{g} M\right) \downarrow_{K \cap 8 H}^{8_{H}} \uparrow_{K \cap 8 H}^{K}(X) .
\end{aligned}
$$

Details are left to the reader.

It is interesting to express the above operations on Mackey functors in terms of the Mackey algebra. In the situation where we have a morphism of rings

$$
f: A \rightarrow B \text {, }
$$

which we do not require to map $1_{A}$ to $1_{B}$, we use the notation

$$
f^{!}: B \text {-mod } \rightarrow A \text {-mod }
$$

for the functor given by restricting the action along $f$. We do here require that modules be unital modules and so if $M$ is a $B$-module then $f^{!}(M)=f\left(1_{A}\right) \cdot M$ by definition, with the $A$-action given via $f$. We use $f_{!}: A$-mod $\rightarrow B$-mod to denote the functor $f_{!}(M)=B \otimes_{A} M$, which is left adjoint to $f^{!}$.

If $H$ is a subgroup of $G$, induction of $H$-sets gives a functor

$$
\uparrow_{H}^{G}: \Omega_{R}(H) \rightarrow \Omega_{R}(G) .
$$

We therefore have an $R$-algebra homomorphism

$$
\alpha: \mu_{R}(H) \rightarrow \mu_{R}(G),
$$

which in terms of symbols is $\alpha\left(I_{K}^{J}\right)=I_{K}^{J}, \alpha\left(R_{K}^{J}\right)=R_{K}^{J}$ and $\alpha\left(c_{h, K}\right)=c_{h, K}$. This morphism is generally not injective as can be seen from the description of the basis elements of $\mu_{R}(G)$, since basis elements in $\mu_{R}(H)$ may become identified by conjugation within $G$.

Similarly, if $N$ is a normal subgroup of $G$ with quotient $Q=G / N$ then there is a functor $\Omega_{R}(Q) \rightarrow \Omega_{R}(G)$ by which we regard a $Q$-set as a $G$-set using the epimorphism $G \rightarrow Q$. Thus there is an $R$-algebra homomorphism

$$
\beta: \mu_{R}(Q) \rightarrow \mu_{R}(G)
$$


given by $\beta\left(I_{K / N}^{J / N}\right)=I_{K}^{J}, \beta\left(R_{K / N}^{J / N}\right)=R_{K}^{J}$ and $\beta\left(c_{h N, K / N}\right)=c_{h, K}$. Since $K$ should contain $N$ here, the latter conjugation is well-defined independently of the choice of $h$ within the coset $h N$.

The third ring homomorphism we will consider is

$$
\gamma: R G \rightarrow \mu_{R}(G)
$$

given by $\gamma(g)=c_{g, 1}$.

(5.4) Lemma. When we identify Mackey functors with $\mu_{R}(G)$-modules we have

(i) $\alpha^{!}(M)=M \downarrow_{H}^{G}$ and $\alpha_{!}(L)=L \uparrow_{H}^{G}$,

(ii) $\beta^{!}(M)$ is the Mackey functor for which $\beta^{!}(M)(H / N)=M(H)$ with induction, restriction and conjugation morphisms inherited from those for $M$, and

(iii) $\gamma^{!}(M)=M(1)$ and $\gamma_{!}(V)=F Q_{V}$.

Proof. We readily identify the restrictions $\alpha^{!}, \beta^{!}$and $\gamma^{!}$as being the stated operations. Now it follows that $\alpha_{!}$and $\gamma_{!}$are as stated because these are known to be the left adjoints of $\alpha^{!}$and $\gamma^{!}$.

From the known adjointness properties of induction, restriction and fixed point functors, we deduce the following corollary.

(5.5) Corollary. (i) $\alpha_{!}$is both right and left adjoint to $\alpha^{!}$.

(ii) $\gamma^{!}$has a right adjoint, namely $V \mapsto F P_{V}$.

The left adjoint $\beta_{!}$of $\beta^{\text {! }}$ is less easy to describe in terms of values on subgroups. Note simply that $\beta$ ! is exact and so $\beta$ ! sends projectives to projectives. Finally we also have:

(5.6) Proposition. Assume that $R=k$ is a field and consider the duality of Section 4. Then $\beta^{!}$has a right adjoint, namely $L \mapsto\left(\beta_{!}\left(L^{*}\right)\right)^{*}$.

Proof. There is a chain of natural bijections

$$
\begin{aligned}
\operatorname{Hom}\left(M,\left(\beta_{!}\left(L^{*}\right)\right)^{*}\right) & \leftrightarrow \operatorname{Hom}\left(\beta_{!}\left(L^{*}\right), M^{*}\right) \\
& \leftrightarrow \operatorname{Hom}\left(L^{*}, \beta^{!}\left(M^{*}\right)\right) \\
& \leftrightarrow \operatorname{Hom}\left(L^{*}, \beta^{!}(M)^{*}\right) \\
& \leftrightarrow \operatorname{Hom}\left(\beta^{!}(M), L\right) .
\end{aligned}
$$

The result follows.

We emphasize that $\beta^{\text {! }}$ and its adjoints are not equal to the other functors relating $\operatorname{Mack}_{R}(G)$ and $\operatorname{Mack}_{R}(Q)$, namely $\operatorname{Inf}_{Q}^{G}$ and its adjoints + and -.

6. DETERMINING THE COMPOSITION FACTORS OF A MACKEY FUNCTOR

In this section we consider Mackey functors over a field $k$, so that the Grothendieck group $G_{0}\left(\operatorname{Mack}_{k}(G)\right)$ has as a basis the isomorphism types of simple Mackey functors over $k$. We may define a map

$$
\psi: G_{0}\left(\operatorname{Mack}_{k}(G)\right) \rightarrow \bigoplus_{\substack{H \leq G \\ \text { uptoconjugacy }}} G_{0}\left(k \bar{N}_{G}(H)\right)
$$


by $M \mapsto(M(H))_{H}$. Here we use the symbol $M$ to denote also the element of $G_{0}\left(\operatorname{Mack}_{k}(G)\right)$ determined by $M$, and likewise $M(H)$ denotes the element of $G_{0}\left(k \bar{N}_{G}(H)\right)$ which this $k \bar{N}_{G}(H)$-module determines.

(6.1) Proposition. $\psi$ is an isomorphism.

Proof. On the left we have the $S_{H, V}$ as a basis, and on the right we have a basis $\sigma_{H, V}$, where $\sigma_{H, V}=V \in G_{0}\left(k \bar{N}_{G}(H)\right)$ is allowed to range over the isomorphism classes of irreducible $k \bar{N}_{G}(H)$-modules. Order both bases in the same way so that the subgroups $H$ appear in non-decreasing order. Then the matrix of $\psi$ is triangular with 1's down the diagonal. Indeed the construction of $S_{H, V}=\left(\operatorname{Inf}_{\bar{N}_{G}(H)}^{N_{G}(H)} S_{1, V}\right) \uparrow_{N_{G}(H)}^{G}$ implies that $S_{H, V}(K)=0$ if $K \searrow_{G} H$, and $S_{H, V}(H)=V$.

The above elementary result gives rise to an algorithm for finding the composition factors of some given Mackey functor $M$, which really amounts to computing the inverse of $\psi$. We suppose we know the modules $M(H)$, and hence the value of $\psi(M)$. Choose a minimal subgroup $H$ for which the component $\psi(M)_{H}$ is non-zero, say

$$
\psi(M)_{H}=\sum_{\text {irreducible } V} \lambda_{V} V
$$

Then $\sum_{\text {irreducible } V} \lambda_{V} S_{H, V}$ occurs in the list of composition factors of $M$, and $\psi\left(M-\sum_{\text {irreducible } V} \lambda_{V} S_{H, V}\right)$ has zero component at $H$. We repeat this process with $M$ replaced by $M-\sum_{\text {irreducible } V} \lambda_{V} S_{H, V}$, gradually working upwards in the poset of subgroups of $G$. This procedure will be demonstrated in Section 15 , where we work with some particular examples.

If we do not need a complete list of composition factors of $M$ we may identify certain composition factors with less work. We recall from Section 2 the notation $\bar{M}(H)=M(H) / \sum_{K<H} I_{K}^{H} M(K)$.

(6.2) Proposition. Let $H \leq G$ and let $V$ be an irreducible $\bar{N}_{G}(H)$-module. Then $S_{H, V}$ occurs as a composition factor of $M$ at least as many times as the multiplicity of $V$ as a composition factor of $\bar{M}(H)$.

Proof. Let $M_{1}$ be the subfunctor of $M$ generated by the modules $M(K)$ for all $K<H$. Then for $J \leq H$, we have $M_{1}(J)=\sum_{K<H, K \leq J} I_{K}^{J}(M(K))$ by 2.4 so that the functor $M_{2}=M / M_{1}$ has $M_{2}(H)=\bar{M}(H)$ and $M_{2}(K)=0$ if $K<_{G} H$. The only composition factors $S_{K, W}$ which are non-zero on $H$ have $K \leq_{G} H$, but these cannot occur as composition factors of $M_{2}$ unless $K={ }_{G} H$, since they are non-zero at $K$. In order to account for all the module composition factors of $M_{2}(H)$ we must have a composition factor $S_{H, V}$ every time $V$ is a composition factor of $M_{2}(H)$. These composition factors of $M_{2}$ are also composition factors of $M$.

Closely related to the last result is the following proposition, which will frequently be used.

(6.3) Proposition. $V$ is a composition factor of $M(1)$ with multiplicity $m$ if and only if $S_{1, V}$ is a composition factor of $M$ with multiplicity $m$.

Proof. Since $S_{H, W}(1)=0$ if $H \neq 1$, only the composition factors indexed by the trivial subgroup can contribute to the evaluation at 1 . But since $S_{1, V}(1)=$ 
$V$, such a simple Mackey functor must occur in $M$ exactly as many times as $V$ occurs in $M(1)$.

In this work we are mainly interested in developing techniques which apply when there may be non-split extensions of Mackey functors, and the methods just presented work in this general situation. To conclude this section we wish to point out that if we happen to be dealing with Mackey functors which are completely reducible (i.e., direct sums of simple Mackey functors) then the bar construction gives complete information. The basis for this is the next lemma.

(6.4) Lemma. Let $S_{H, V}$ be a simple Mackey functor. Then

$$
\overline{S_{H, V}}(K) \cong \begin{cases}V & \text { if } H \text { and } K \text { are conjugate, } \\ 0 & \text { otherwise. }\end{cases}
$$

Proof. $S_{H, V}(K)=0$ unless $K \geq_{G} H$, and if $K>_{G} H$, then since $S_{H, V}$ is generated by $S_{H, V}(H)$ (by Proposition 2.3 of [20]), we have

$$
S_{H, V}(K)=\sum_{J<K} I_{J}^{K} S_{H, V}(J)
$$

using 2.4. It follows that $\overline{S_{H, V}}(K)=0$. Finally $\overline{S_{H, V}}(H)=S_{H, V}(H)=V$ since $S_{H, V}$ vanishes on proper subgroups of $H$.

The first part in the next corollary works for any completely reducible Mackey functor $M$.

(6.5) Corollary. Suppose $M$ is a Mackey functor over a field $k$ in which $|G|$ is invertible. Then

(i) $M \cong \bigoplus_{(H, V)} n_{H, V} S_{H, V}$ where $n_{H, V}$ denotes the multiplicity of $V$ as a composition factor of $\bar{M}(H)$ when regarded as a $k \bar{N}_{G}(H)$-module.

(ii) $M \cong \bigoplus_{H \text { up to conjugacy }}\left(\operatorname{Inf}_{\bar{N}(H)}^{N(H)} F P_{\bar{M}(H)}\right) \uparrow_{N(H)}^{G}$.

Proof. (i) Certainly there is some isomorphism of the type claimed, since $M$ is completely reducible by 3.5 , and it remains to determine the multiplicities $n_{H, V}$. Taking the bar of both sides of the isomorphism at $H$, all terms on the right disappear except for the simples associated to $H$, and for each of those we obtain a contribution $V$. Thus $\bar{M}(H) \cong \bigoplus_{V} n_{H, V} V$ for each subgroup $H$, and hence $n_{H, V}$ is the multiplicity as claimed.

(ii) We group together all the terms of the direct sum in (i) which correspond to a fixed subgroup $H$. When $|G|$ is invertible each simple has the form

$$
S_{H, V}=\left(\operatorname{Inf}_{\bar{N}(H)}^{N(H)} F P_{V}\right) \uparrow_{N(H)}^{G}
$$

by [20], and when we take the direct sum of these over the simple summands of $\bar{M}(H)$ we obtain the desired result.

There is no mention of any map which will achieve the isomorphism in the last result, and we now go some way to set this right, recovering a result of the first author [19]. For any Mackey functor $M$ and subgroup $H$ of $G$ the Brauer morphism $\beta_{H}$ is defined to be the composite

$$
\beta_{H}: M(G) \stackrel{R_{H}^{G}}{\longrightarrow} M(H) \rightarrow \bar{M}(H)
$$

where the last morphism is the natural quotient map. 
(6.6) Theorem (Thévenaz [19]). Let $M$ be a Mackey functor over a field in which $|G|$ is invertible. Then

$$
\bigoplus \beta_{H}: M(G) \rightarrow \bigoplus_{\substack{H \leq \leq G \\ \text { uproconjugacy }}} \bar{M}(H)^{N_{G}(H)}
$$

is an isomorphism.

Proof. Notice first that since all conjugations act as the identity on $M(G)$, the image of each $\beta_{H}$ is indeed contained in the fixed points under $N_{G}(H)$.

We replace $M$ by its decomposition $M \cong \bigoplus n_{H, V} S_{H, V}$. Since each $\beta_{H}$ is compatible with this direct sum decomposition, it suffices to prove the result when $M=S_{H, V}$. Now $\overline{S_{H, V}}$ is non-zero only on the subgroup $H$, so it suffices to show that $\beta_{H}: S_{H, V}(G) \rightarrow \overline{S_{H, V}}(H)^{N_{G}(H)}$ is an isomorphism. We defined $\beta_{H}$ as the composite

$$
\beta_{H}: S_{H, V}(G) \stackrel{R_{H}^{G}}{\longrightarrow} S_{H, V}(H) \rightarrow \overline{S_{H, V}}(H)
$$

but in this case the final quotient map is an isomorphism, so we are reduced to showing that

$$
R_{H}^{G}: S_{H, V}(G) \rightarrow S_{H, V}(H)^{N_{G}(H)}
$$

is an isomorphism. In $G$-set notation this map is

$$
S_{H, V}^{G}(G / G) \rightarrow S_{H, V}^{G}(G / H)
$$

and since $S_{H, V}$ is induced from $N_{G}(H)$ this is the same as

$$
S_{H, V}^{N_{G}(H)}\left(G / G \downarrow_{N_{G}(H)}^{G}\right) \rightarrow S_{H, V}^{N_{G}(H)}\left(G / H \downarrow_{N_{G}(H)}^{G}\right) .
$$

Using the decomposition of the final term above, this is

$$
\begin{aligned}
\bigoplus_{x \in\left[N_{G}(H) \backslash G / H\right]} R_{N_{G}(H) \cap x_{H}}^{N_{G}(H)}: S_{H, V}^{N_{G}(H)}\left(N_{G}(H)\right) & \\
& \longrightarrow \bigoplus_{x \in\left[N_{G}(H) \backslash G / H\right]} S_{H, V}^{N_{G}(H)}\left(N_{G}(H) \cap{ }^{x} H\right) .
\end{aligned}
$$

There is only one non-zero term on the right, namely $S_{H, V}^{N_{G}(H)}(H)$. We identify $S_{H, V}^{N_{G}(H)}$ as $\operatorname{Inf}_{\bar{N}_{G}(H)}^{N_{G}(H)} F P_{V}$, and now the restriction map becomes

$$
R_{H}^{N_{G}(H)}: \operatorname{Inf}_{\bar{N}_{G}(H)}^{N_{G}(H)} F P_{V}\left(N_{G}(H)\right) \rightarrow \operatorname{Inf}_{\bar{N}_{G}(H)}^{N_{G}(H)} F P_{V}(H),
$$

which is the inclusion of fixed points. This establishes the desired isomorphism for the simple functor $S_{H, V}$, thus completing the proof.

As an example of how the theory we have developed may be applied, we give the Mackey functor decomposition of the character ring of $G$, thus extending the result due to Puig which is presented in [19]. We let $R(G)$ denote the ring of complex characters of $G$, taken with rational coefficients. For each cyclic subgroup $H \leq G$ we let $\zeta_{|H|}$ denote a primitive complex $|H|$ root of unity. By identifying the cyclic group $\left\langle\zeta_{|H|}\right\rangle$ with the group of characters of $H$, we obtain an action of $N_{G}(H)$ on $\mathbb{Q}\left(\zeta_{|H|}\right)$, so that $\mathbb{Q}\left(\zeta_{|H|}\right)$ becomes a $\mathbb{Q} \bar{N}_{G}(H)$-module. 
(6.7) Example. (i) As a Mackey functor we have the decompositions

$$
R \cong \bigoplus_{\substack{(H, V) \\
H \text { cyclic }}} n_{H, V} S_{H, V} \cong \bigoplus_{\begin{array}{c}
\text { cyclic } H \leq G \\
\text { uptoc conjugacy }
\end{array}}\left(\operatorname{Inf}_{\bar{N}(H)}^{N(H)} F P_{Q\left(\zeta_{|H|}\right)}\right) \uparrow_{N(H)}^{G}
$$

where $n_{H, V}$ is the multiplicity of $V$ in $\mathbb{Q}\left(\zeta_{|H|}\right)$ as a $\mathbb{Q} \bar{N}_{G}(H)$-module.

(ii) (Puig) The Brauer morphisms give an isomorphism

$$
\bigoplus \beta_{H}: R(G) \rightarrow \bigoplus_{\substack{\text { cyclic } H \leq G \\ \text { uptoc conjugacy }}} \mathbb{Q}\left(\zeta_{|H|}\right)^{N_{G}(H)}
$$

Proof. As explained in [19], $\bar{R}(H)=0$ if $H$ is not cyclic, by Artin's induction theorem, and if $H$ is cyclic then $\bar{R}(H) \cong \mathbb{Q}\left(\zeta_{|H|}\right)$ (a result of Puig). The result follows from 6.5 and 6.6 .

It is interesting to note in the above example that if we are only interested in the group structure of $R(G)$ then the trivial summands of $\mathbb{Q}\left(\zeta_{|H|}\right)$ give sufficient information, whereas the structure of $R$ as a Mackey functor requires information about all the summands.

\section{DECOMPOSITION THEORY}

Let $\mathscr{O}$ be a complete discrete valuation ring with quotient field $K$ of characteristic 0 and residue field $k$ of characteristic $p$. In this situation the Mackey algebra $\mu_{\mathscr{O}}(G)$ is an $\mathscr{O}$-order in the finite dimensional semisimple $K$-algebra $\mu_{K}(G)$, because it is free as an $\mathscr{O}$-module by 3.2 . There is a decomposition theory exactly analogous to the one for group representations which relates the decomposition map to the Cartan matrix of $\mu_{k}(G)$. We summarise the ingredients in this theory, for which a possible reference is [8, Section 48]. Although we are really dealing with Mackey functors, to make it clear how the results here are instances of the existing theory we will refer to the Mackey functors as $\mu_{K}(G)$-modules, $\mu_{\mathscr{O}}(G)$-modules, etc.

Every $\mu_{K}(G)$-module $M$ contains a $\mu_{\mathscr{O}}(G)$-lattice $M_{\ell}$ whose $K$-span is $M$. It is a theorem of Brauer that the set of composition factors of $k \otimes_{\mathcal{O}} M_{\ell}$ (taken with multiplicities) is independent of the choice of lattice $M_{\ell}$, and so we may define for each simple $\mu_{K}(G)$-module $S$ the numbers

$$
\begin{aligned}
d_{S T}= & \text { the multiplicity of the simple } \mu_{k}(G) \text {-module } T \\
& \text { as a composition factor in } k \otimes_{\mathscr{O}} S_{\ell} .
\end{aligned}
$$

These numbers are the entries in the decomposition matrix $D=\left(d_{S T}\right)$. For each simple $\mu_{k}(G)$-module $T$ let $P_{T}$ denote the projective cover of $T$. The Cartan matrix of $\mu_{k}(G)$ is the square matrix $C=\left(c_{T U}\right)$ with entries indexed by simple $\mu_{k}(G)$-modules $T, U$, where

$$
c_{T U}=\text { the multiplicity of } T \text { as a composition factor in } P_{U} \text {. }
$$

We denote by $\hat{P}_{T}$ the projective cover as a $\mu_{\mathscr{O}}(G)$-module of the simple module $T$, so the reduction of $\hat{P}_{T} \operatorname{modulo} \operatorname{Rad}(\mathscr{O})$ is $P_{T}$. We now quote the following result of Brauer. 
(7.1) Theorem. Suppose that $K$ is a splitting field for $\mu_{K}(G)$ and $k$ is a splitting field for $\mu_{k}(G)$. Then

(i) $C=D^{T} D$, where $D^{T}$ denotes the transpose of $D$,

(ii) $K \otimes_{\mathscr{O}} \hat{P}_{T}$ is a direct sum of simple $\mu_{K}(G)$-modules in which $S$ appears with multiplicity $d_{S T}$.

(7.2) Corollary. The Cartan matrix of Mackey functors over $k$ is symmetric.

We now show how to compute the Mackey functor decomposition matrix in a practical way by relating it to the decomposition theory for group representations using the work of the last section. In the process we show that the decomposition map for Mackey functors is surjective, and hence that the Car$\tan$ matrix is non-singular. The method depends on the observation that the mapping $\psi$ which we introduced in Section 6 is compatible with decomposition in the following sense. We regard the decomposition for Mackey functors as a homomorphism

$$
d: G_{0}\left(\operatorname{Mack}_{K}(G)\right) \rightarrow G_{0}\left(\operatorname{Mack}_{k}(G)\right) .
$$

We also have for each subgroup $H \leq G$ the group-theoretic decomposition

$$
d: G_{0}\left(K \bar{N}_{G}(H)\right) \rightarrow G_{0}\left(k \bar{N}_{G}(H)\right) .
$$

(7.3) Proposition. The square

commutes.

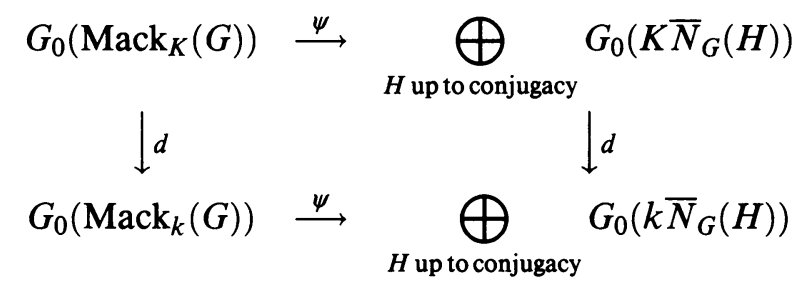

Proof. On applying the composite around either side of the square to an element in $G_{0}\left(\operatorname{Mack}_{K}(G)\right)$ represented by a Mackey functor $M$, we obtain as component at the subgroup $H$ the term $k \otimes_{\mathscr{O}} M(H)_{\ell}$.

To compute the decomposition map in particular cases using this approach one first determines the two mappings $\psi$, for Mackey functors over $K$ and over $k$. Now to compute the decomposition of a Mackey functor $M$ over $K$ one finds the decomposition of the module $M(H)$ for each subgroup $H \leq G$. This gives a set of simple $k \bar{N}_{G}(H)$-modules as composition factors, and now by inverting $\psi$ for Mackey functors over $k$ we obtain a set of simple Mackey functors whose direct sum evaluated at $H$ also has these simple $k \bar{N}_{G}(H)$-modules as composition factors. These simple Mackey functors are the composition factors of the decomposition of $M$. The tables we give of decomposition matrices were all computed in this way.

Before deducing a corollary we remind the reader of the following elementary fact from linear algebra.

(7.4) Lemma. For any matrix $D$ we have $\operatorname{rank} D^{T} D=\operatorname{rank} D$.

Proof. We show that the bilinear form associated to $D^{T} D$ has rank equal to the rank of $D$. This form is defined on column vectors by $\langle x, y\rangle=x^{T} D^{T} D y$. 
Evidently its rank can be no greater than that of $D$. On the other hand the restriction of the bilinear form to any complement of the kernel of $D$ is nonsingular, which proves the result.

(7.5) Corollary. The decomposition map for Mackey functors is surjective; hence the Cartan matrix is non-singular.

Proof. Surjectivity follows from the proposition because $\psi$ is an isomorphism and the decomposition maps for the group rings are known to be surjective [6, (21.16)]. Non-singularity of the Cartan matrix now follows, using the last lemma and Theorem 7.1.

Contrary to the case of group algebras, it should be noted that the determinant of the Cartan matrix is usually not a power of $p$.

\section{BURNSIDE MACKey FUNCTORS}

In $\mu_{R}(G)$ we have an obvious expression

$$
1=\sum_{\substack{H \leq G \\ \text { up to conjugacy }}} I_{H}^{H}
$$

which gives the identity as a sum of mutually orthogonal idempotents. We thus have a decomposition

$$
\mu_{R}(G)=\bigoplus_{\substack{H \leq G \\ \text { up toconjugacy }}} \mu_{R}(G) \cdot I_{H}^{H}
$$

where the $\mu_{R}(G) \cdot I_{H}^{H}$ are projective $\mu_{R}(G)$-modules. In this section we identify the projective Mackey functors to which they correspond in terms of the Burnside ring.

We denote by $B(G)$ the Burnside ring (over $R$ ) of $G$. Recall that this is the free $R$-module with basis the $G$-sets $G / H$ where $H$ is taken up to conjugacy. By means of induction, restriction and conjugation of $G$-sets this gives rise to a Mackey functor denoted $B^{G}$, which we call the Burnside Mackey functor for $G$ (or just Burnside functor). Its definition is that $B^{G}(H)=B(H)$, and it should not be confused with the Burnside ring, since it is a Mackey functor. In fact, $B^{G}$ has more than just the structure of a Mackey functor, it is a Green functor, meaning that each $B^{G}(H)$ is a ring satisfying certain axioms [9].

In the next result we will use the fact that $B^{G}$ is generated as a Mackey functor by the single element $G / G \in B^{G}(G)$, since any $H / K \in B^{G}(H)$ may be written $H / K=I_{K}^{H} R_{K}^{G}(G / G)$.

(8.1) Proposition. The $\mu_{R}(G)$-module which corresponds to $B^{G}$ is isomorphic to $\mu_{R}(G) \cdot I_{G}^{G}$. This has as an $R$-basis the symbols $I_{K}^{H} R_{K}^{G}$ where $K$ is a subgroup of $H$ determined up to $H$-conjugacy, and $H$ ranges over subgroups of $G$. In particular, $B^{G}$ is a projective Mackey functor.

Proof. We first verify that $\mu_{R}(G) \cdot I_{G}^{G}$ has the stated basis. For a typical basis element $I_{K}^{H} c_{g} R_{K^{g}}^{J}$ of $\mu_{R}(G)$ the product with $I_{G}^{G}$ on the right is zero unless $J=G$ and then $I_{K}^{H} c_{g} R_{K g}^{G} I_{G}^{G}=I_{K}^{H} R_{K}^{G} c_{g}=I_{K}^{H} R_{K}^{G}$ since $c_{g}$ acts trivially at the level of $G$. Thus $\mu_{R}(G) \cdot I_{G}^{G}$ is the $R$-span of the stated elements, and they are linearly independent because they form part of the basis of $\mu_{R}(G)$. 
By abuse of notation we will use the same symbol $B^{G}$ to denote both the Burnside Mackey functor, and also the $\mu_{R}(G)$-module which corresponds to it. There is a unique map of $\mu_{R}(G)$-modules $\mu_{R}(G) \rightarrow B^{G}$ which sends $I_{G}^{G} \mapsto$ $G / G, I_{H}^{H} \mapsto 0$ if $H \neq G$. This is surjective since $G / G$ generates $B^{G}$. A basis element $I_{K}^{H} R_{K}^{G}$ is mapped to $I_{K}^{H} R_{K}^{G}(G / G)=H / K$, and since these images are linearly independent the map is an isomorphism on restriction to $\mu_{R}(G) \cdot I_{G}^{G}$.

We can interpet this identification of $B^{G}$ with $\mu_{R}(G) \cdot I_{G}^{G}$ in terms of an adjointness property. Consider the composite functor

$$
\operatorname{Mack}_{R}(G) \rightarrow R \text {-mod } \rightarrow \text { Set }
$$

where we first evaluate a Mackey functor $M$ at $G$, and then forget the $R$ module structure to obtain a set. We have also a functor in the opposite direction: for any one-point set $*$, we may speak of the Burnside Mackey functor generated by $*$, this being a copy of $B^{G}$ with $*$ corresponding to $G / G \in B^{G}(G)$.

(8.2) Corollary. (i) Let $M$ be a Mackey functor and $m \in M(G)$. Then there exists a unique morphism of Mackey functors $B^{G} \rightarrow M$ whose evaluation at $G$ maps $G / G$ to $m$. Thus the functor Set $\rightarrow \operatorname{Mack}_{R}(G)$ which sends a set to the direct sum of the $B^{G}$ generated by its elements is left adjoint to the functor $\operatorname{Mack}_{R}(G) \rightarrow$ Set which sends $M \mapsto M(G)$.

(ii) $\operatorname{End}_{\operatorname{Mack}_{R}(G)}\left(B^{G}\right) \cong B(G)$.

Proof. (i) In terms of the $\mu_{R}(G)$-module $\mu_{R}(G) \cdot I_{G}^{G}$ the morphism is the one which extends the assignment $I_{G}^{G} \mapsto m$.

(ii) For each $X \in B(G)$ let $\hat{X}: B^{G} \rightarrow B^{G}$ be the unique endomorphism of $B^{G}$ which sends $G / G$ to $X$. For any $G$-set $G / H$ we may write $G / H=$ $I_{H}^{G} R_{H}^{G}(G / G)$ so that

$$
\hat{X}(G / H)=\hat{X}\left(I_{H}^{G} R_{H}^{G}(G / G)\right)=I_{H}^{G} R_{H}^{G} \hat{X}(G / G)=I_{H}^{G} R_{H}^{G}(X)=X \cdot G / H,
$$

this last product being the multiplication in $B(G)$. By extending this to combinations of the basis elements in $B(G)$ we see that for any $Y \in B(G)$ we have $\hat{X}(Y)=X \cdot Y$. Now

$$
\hat{X} \hat{Y}(G / G)=\hat{X}(Y)=X \cdot Y=\widehat{X \cdot Y}(G / G)
$$

so that $\hat{X} \hat{Y}=\widehat{X \cdot Y}$. Thus $\wedge: B(G) \rightarrow \operatorname{End}_{\operatorname{Mack}_{R}(G)}\left(B^{G}\right)$ is a ring homomorphism. It has a 2-sided inverse given by the assignment

$$
\begin{aligned}
\operatorname{End}_{\operatorname{Mack}_{R}(G)}\left(B^{G}\right) & \rightarrow B(G), \\
\phi & \mapsto \phi(G)(G / G)
\end{aligned}
$$

which we may see by using the fact that an endomorphism $\phi$ is completely determined by the value of $\phi(G)(G / G)$.

We now come to the general description of the projective Mackey functors given by the idempotents $I_{H}^{H}$. First we note that regarding Mackey functors as additive functors $\Omega_{R}(G) \rightarrow R$-mod we may consider the representable functors $\operatorname{Hom}_{\Omega_{R}(G)}(G / H, \quad)$, which are projective by Yoneda's lemma. 
(8.3) Theorem. The projective Mackey functors given (in different notations) by $B^{H} \uparrow_{H}^{G}, \mu_{R}(G) \cdot I_{H}^{H}$ and $\operatorname{Hom}_{\Omega_{R}(G)}(G / H, \quad)$ are isomorphic.

Proof. One first sees that $\mu_{R}(G) \cdot I_{H}^{H}$ and $\operatorname{Hom}_{\Omega_{R}(G)}(G / H$, ) give the same Mackey functor because their evaluation at a subgroup $K$ is

$$
I_{K}^{K} \cdot \mu_{R}(G) \cdot I_{H}^{H}=\text { the } R \text {-span of }\left\{I_{g_{L}}^{K} c_{g} R_{L}^{H} \mid g \in G, L \leq H \cap K^{g}\right\}
$$

for $\mu_{R}(G) \cdot I_{H}^{H}$, and for $\operatorname{Hom}_{\Omega_{R}(G)}(G / H$, ) it is the $R$-span of all diagrams

$$
G / K \stackrel{\pi_{g_{L}}^{K} c_{g}}{\longleftarrow} G / L \stackrel{\pi_{L}^{H}}{\longrightarrow} G / H
$$

These $R$-modules are seen to be isomorphic using the isomorphisms introduced in 3.3. Furthermore these isomorphisms are natural, that is they commute with the operations of induction, restriction and conjugation, so we have an isomorphism of Mackey functors.

We show that $B^{H} \uparrow_{H}^{G}$ and $\operatorname{Hom}_{\Omega_{R}(G)}(G / H, \quad)$ give the same Mackey functor by first observing that $\operatorname{Hom}_{\Omega_{R}(G)}(G / H$,$) is induced from H$. In fact, for a $G$-set $X$ we have

$$
\operatorname{Hom}_{\Omega_{R}(G)}(G / H, X) \cong \operatorname{Hom}_{\Omega_{R}(H)}\left(H / H, X \downarrow_{H}^{G}\right)
$$

which is the same as $\left(\operatorname{Hom}_{\Omega_{R}(H)}(H / H, \quad)\right) \uparrow_{H}^{G}$ evaluated at $X$. Thus it suffices to show that $B^{H} \cong \operatorname{Hom}_{\Omega_{R}(H)}(H / H, \quad)$. But we already know from 8.1 that $B^{H}$ gives the same Mackey functor as $\mu_{R}(H) \cdot I_{H}^{H}$, and we just showed that this is the same as $\operatorname{Hom}_{\Omega_{R}(H)}(H / H, \quad)$.

(8.4) Corollary. (i) There is an isomorphism of Mackey functors

$$
\mu_{R}(G) \cong \bigoplus_{H \text { up to conjugacy }} B^{H} \uparrow_{H}^{G} .
$$

(ii) Any Mackey functor is isomorphic to a quotient of a direct sum of induced Burnside functors.

(8.5) Corollary. $\operatorname{dim} B^{H} \uparrow_{H}^{G}(K)=\operatorname{dim} B^{K} \uparrow_{K}^{G}(H)$.

Proof. The left-hand side is the dimension of $I_{K}^{K} \cdot \mu_{R}(G) \cdot I_{H}^{H}$, which has a basis

$$
\left\{I_{{ }_{s}}^{K} c_{g} R_{L}^{H} \mid g \in[K \backslash G / H], L \leq H \cap K^{g} \text { up to } H \cap K^{g} \text {-conjugacy }\right\} .
$$

Evidently $I_{H}^{H} \cdot \mu_{R}(G) \cdot I_{K}^{K}$ has a similar basis with $H$ and $K$ reversed. There is a bijection between these two bases.

For the rest of this section we specialize to the case where the coefficient ring $R$ is a field $k$. The Burnside functors are in general not themselves indecomposable projectives. To recall our notation, we denote by $P_{H, V}$ the projective cover of the simple Mackey functor $S_{H, V}$. There is then a primitive idempotent $e_{H, V} \in \mu_{k}(G)$ for which $P_{H, V} \cong \mu_{k}(G) e_{H, V}$. We now give the exact decomposition of $B^{H} \uparrow_{H}^{G}$ into indecomposable projectives.

(8.6) Theorem. Let $k$ be a field which is a splitting field for $\mu_{k}(G)$. Then the multiplicity of $P_{H, V}$ as a summand of $B^{K} \uparrow_{K}^{G}$ is $\operatorname{dim} S_{H, V}(K)$; that is

$$
B^{K} \uparrow_{K}^{G} \cong \bigoplus_{(H, V)} \operatorname{dim} S_{H, V}(K) \cdot P_{H, V}
$$


Proof. We may write $I_{K}^{K}$ as a sum of orthogonal idempotents $I_{K}^{K}=\sum E_{H, V}$ where each $E_{H, V}$ is an orthogonal sum of primitive idempotents all conjugate to $e_{H, V}$. Since $B^{K} \uparrow_{K}^{G} \cong \mu_{k}(G) I_{K}^{K}$, we have to show for each pair $(H, V)$ that the number of idempotents in this decomposition of $E_{H, V}$ is $\operatorname{dim} S_{H, V}(K)$. We use the fact that $\operatorname{dim} e_{H, V} S_{H, V}=1$, while $\operatorname{dim} e_{J, W} S_{H, V}=0$ if $(J, W) \neq$ $(H, V)$. This number of idempotents is $\operatorname{dim} E_{H, V} S_{H, V}=\operatorname{dim} I_{K}^{K} S_{H, V}=$ $\operatorname{dim} S_{H, V}(K)$, as required.

Remark. By lifting idempotents, or by lifting projective modules, the theorem also holds over a coefficient ring which is a complete local ring.

(8.7) Corollary. Let $k$ be a field which is a splitting field for $\mu_{k}(G)$. Then

(i) $P_{H, V}$ cannot be a summand of $B^{K} \uparrow_{K}^{G}$ unless $H$ is conjugate to a subgroup of $K$, and

(ii) for every simple $k \bar{N}_{G}(K)$-module $V, P_{K, V}$ is a summand of $B^{K} \uparrow_{K}^{G}$ with multiplicity $\operatorname{dim} V$.

Proof. (i) $S_{H, V}(K)=0$ unless $H$ is conjugate to a subgroup of $K$.

(ii) $S_{K, V}(K)=V$.

The numbers $\operatorname{dim} S_{H, V}(K)$ in Theorem 8.6 may be computed quite explicitly in terms of the simple module $V$ and the fusion within $G$. We now show how this may be done and draw some corollaries. We use the notion of the carrier of $H$ into $K$, which is the set $T(H, K)=\left\{g \in G \mid{ }^{g} H \subseteq K\right\}$.

(8.8) Proposition. $S_{H, V}(K)=\underset{g \in\left[K \backslash T(H, K) / N_{G}(H)\right]}{\operatorname{tr}_{1}^{N_{K}(H) / H}(V) .}$

The indexing set in the sum bijects with the set of $G$-conjugates ${ }^{g} H$ contained in $K$, taken up to $K$-conjugacy. If we are interested in $\operatorname{dim} S_{H, V}(K)$

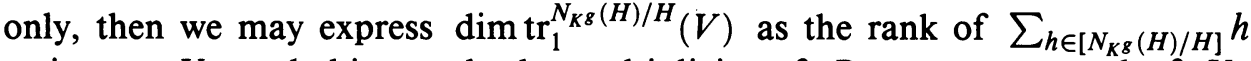
acting on $V$, and this equals the multiplicity of $P_{k}$ as a summand of $V$, where $P_{k}$ is the projective cover of $k$ as a $k\left[N_{K^{g}}(H) / H\right]$-module.

Proof. From [20] we have $S_{H, V}=S_{H, V}^{G}=S_{H, V}^{N_{G}(H)} \uparrow_{N_{G}(H)}^{G}$ and so

$$
S_{H, V}(K)=\bigoplus_{g \in\left[K \backslash G / N_{G}(H)\right]} S_{H, V}^{N_{G}(H)}\left(K^{g} \cap N_{G}(H)\right) .
$$

Now the terms in the sum are zero unless $H \subseteq K^{g}$, so $g \in T(H, K)$, and

$$
S_{H, V}^{N_{G}(H)}\left(K^{g} \cap N_{G}(H)\right)=S_{1, V}^{\bar{N}_{G}(H)}\left(N_{K^{g}}(H) / H\right)
$$

is the image of the trace from the identity as stated.

(8.9) Corollary. Let $k$ be a field which is a splitting field for $\mu_{k}(G)$.

(i) If char $k=0$ then

$$
B^{G} \cong \bigoplus_{H \leq G, \text { up to conjugacy }} P_{H, k} .
$$


(ii) If char $k=p$ then

$$
B^{G} \cong \bigoplus_{\substack{H \text { pltoconjugec } \\ p \nmid\left|N_{G}(H): H\right|}} P_{H, k}
$$

In particular $B^{G}$ is indecomposable if and only if $G$ is a p-group, in which case $B^{G}$ is the projective cover of $S_{G, 1}$.

Proof. We first prove (ii). Here the multiplicity of $P_{H, V}$ is $\operatorname{dim} \operatorname{tr}_{1}^{\bar{N}_{G}(H)}(V)$, which is the number of times $P_{k}$ as a $\bar{N}_{G}(H)$-module is a summand of the simple module $V$. If this number is non-zero then $P_{k}$ must itself be simple and equal to $V$, so $V=k$ and this is a projective module. This means that $\bar{N}_{G}(H)$ is a $p^{\prime}$-group and the multiplicity is 1 .

(i) The argument is the same as with (ii), except that now $k$ is always a projective module and so every subgroup $H$ occurs.

Combining 8.9 with 8.2(ii) this turns out to give a proof of part of a wellknown result, which we quote later as 9.3. In characteristic 0 this is due to Burnside and Solomon, and to Dress in the case of characteristic $p$.

(8.10) Corollary. If char $k=0$ the primitive idempotents in $B(G)$ biject with the conjugacy classes of all subgroups of $G$. If char $k=p$ the primitive idempotents in $B(G)$ biject with the conjugacy classes of subgroups $H$ for which $\bar{N}_{G}(H)$ is a $p^{\prime}$-group.

\section{DeCOMpositions INDUCED BY THE BURNSIDE RING}

It is well-known that the Burnside ring $B(G)$ acts as a ring of endomorphisms of every Mackey functor for $G$. Explicitly, if $M$ is a Mackey functor and $Z$ is a $G$-set, then the action of $Z$ is defined as the natural transformation

$$
M(X) \stackrel{M^{*}\left(\mathrm{pr}_{2}\right)}{\longrightarrow} M(Z \times X) \stackrel{M_{*}\left(\mathrm{pr}_{2}\right)}{\longrightarrow} M(X),
$$

where $X$ is an arbitrary $G$-set and $\operatorname{pr}_{2}: Z \times X \rightarrow X$ is the second projection. Using the axiom on pullbacks in the second definition of Mackey functors, it easy to see that the action of $B(G)$ on $M$ is indeed as a ring of Mackey functor endomorphisms.

In the special case where $Z=G / H$, we have an isomorphism

$$
G / H \times X \cong G \times{ }_{H} X=X \downarrow_{H}^{G} \uparrow_{H}^{G}
$$

mapping $(g H, x)$ to $\left(g, g^{-1} x\right)$. Moreover the map $p r_{2}$ corresponds to the natural map

$$
\alpha: G \times_{H} X \rightarrow X, \quad \alpha(g, x)=g x .
$$

Thus if we set $\theta_{H}=M_{*}(\alpha)$ and $\theta^{H}=M^{*}(\alpha)$, then in the action of the Burnside ring on $M$, the transitive $G$-set $G / H$ acts as $\theta_{H} \theta^{H}$.

We may describe this action of the Burnside ring in yet another way which uses the induction and restriction maps. On evaluation at the subgroup $K$ (i.e. at the $G$-set $G / K)$, the map

$$
\theta_{H}(K): \bigoplus_{g \in[K \backslash G / H]} M\left(K \cap{ }^{g} H\right) \rightarrow M(K)
$$


is equal to the sum of the inductions $I_{K \cap B H}^{K}$, and similarly $\theta^{H}$ is the sum of the restrictions $R_{K \cap 8 H}^{K}$. Therefore the action of $G / H$ on $x \in M(K)$ is

$$
G / H \cdot x=\sum_{g \in[K \backslash G / H]} I_{K \cap 8 H}^{K} R_{K \cap 8 H}^{K}(x)
$$

and this assignment at the level of the subgroup $K$ gives a natural transformation of Mackey functors $M \rightarrow M$. One may describe this by saying that if $x \in M(G)$ then $G / H$ acts as $I_{H}^{G} R_{H}^{G}$, and if $x \in M(K)$ then $G / H$ acts as the $K$-set $G / H \downarrow_{K}^{G}$. This action is closely related to the structure of $M$ as a Green module over the Green functor $B^{G}$, although the two should not be confused since the Burnside ring regarded as $B^{G}(G)$ acts only on $M(G)$ in the Green module structure, whereas in the action we are now describing $B(G)$ acts on every $R$-module $M(K)$. The connection between these two structures is that the Green module structure provides us with actions of $B(K)$ on $M(K)$ for every $K \leq G$. Now composing with the restriction $B(G) \rightarrow B(K)$, which is a ring homomorphism, gives the action of $B(G)$ on $M(K)$.

As a consequence of the action of the Burnside ring we have the following well-known result.

(9.1) Proposition. Every expression in $B(G)$ of the identity as a sum of orthogonal idempotents

$$
1=\sum_{i} e_{i}
$$

gives rise to a decomposition $M=\bigoplus e_{i} \cdot M$ of every Mackey functor $M$.

Here we write $e_{i} \cdot M$ for the image of $M$ under the endomorphism $e_{i}$.

It is an immediate interpretation of the above decomposition that it provides a partial separation of Mackey functors into different blocks. To make it transparent that the notion of block we have in mind is the same as the usual one in the representation theory of algebras, we interpret the action of the Burnside ring in terms of the Mackey algebra. To do this we observe that $B(G)$ acts via elements of the Mackey algebra, and since the action commutes with all the Mackey functor operations these elements must be central. This is the basis of the proof of the following result.

(9.2) Proposition. There is a central subring of $\mu_{R}(G)$ isomorphic to the Burnside ring $B(G)$ and which contains the identity. Specifically, this subring has a basis consisting of elements

$$
b_{H}=\sum_{K \leq G} \sum_{x \in[K \backslash G / H]} I_{K \cap x_{H}}^{K} R_{K \cap x_{H}}^{K} .
$$

Proof. In the action of the Burnside ring on a Mackey functor $M$ the $G$-set $G / H$ acts precisely as $b_{H}$. Indeed since $M(J)=I_{J}^{J} \cdot M$, the only term in the first sum which does not act by zero on $M(J)$ is the one corresponding to $K=J$, and we have seen in our preliminary remarks that the action is as stated. In particular this holds for the action on the regular representation $\mu_{R}(G)$ and so the assignment

$$
B(G) \rightarrow \mu_{R}(G), \quad G / H \mapsto b_{H}
$$

does extend by linearity to a ring homomorphism. It is injective since the $b_{H}$ are linearly independent, which follows from the fact that the action of the 
span of these elements on $B(G)=B^{G}(G) \cong\left(\mu_{R}(G) I_{G}^{G}\right)(G)=I_{G}^{G} \mu_{R}(G) I_{G}^{G}$ is the regular representation of the Burnside ring on itself, namely $b_{H}$ acts as $G / H$. In this action the $b_{H}$ are independent. Finally the $b_{H}$ are central since in the regular representation of $\mu_{R}(G)$ they act as Mackey functor endomorphisms of $\mu_{R}(G)$, and so commute in their action with all of $\mu_{R}(G)$. Since the regular representation is faithful, the $b_{H}$ must themselves be central.

In view of this result every idempotent of $B(G)$ gives a central idempotent of $\mu_{R}(G)$, and hence a decomposition of the Mackey algebra into ring direct summands. We will see later that we do not in general obtain primitive central idempotents of $\mu_{R}(G)$ in this way, so that we have a decomposition into unions of blocks, rather than blocks themselves. For the moment we can at least say that if some Burnside idempotent is non-zero on one indecomposable Mackey functor and zero on another then the two Mackey functors lie in different blocks. Later on in Section 17 we will give the exact decomposition into blocks. It will follow also that the centre of $\mu_{R}(G)$ is bigger than $B(G)$ because primitive idempotents of the Burnside ring decompose further as orthogonal sums of central primitive idempotents in $\mu_{R}(G)$.

We now examine more closely the particular structure of the Burnside ring idempotents and quote the following result. We say that a group $J$ is $p$ perfect if there is no proper normal subgroup $N$ of $J$ with $J / N$ a $p$-group, that is, $O^{p}(J)=J$. It is evident that for any group $H$, the only normal $p$ perfect subgroup of $H$ with $p$-power index is $O^{p}(H)$, and given a $p$-perfect subgroup $J$ of a group $G$, the subgroups $H \leq G$ with $O^{p}(H)=J$ are precisely the subgroups $H \leq N_{G}(J)$ with $J \leq H$ and $H / J$ a $p$-group.

\section{(9.3) Theorem.}

(i) (Burnside, Solomon) Let $\mathbb{Z}[1 /|G|]$ be the subring of $\mathbb{Q}$ in which only the prime divisors of $|G|$ are inverted. Then in the Burnside algebra $B(G)$ over $\mathbb{Z}[1 /[G]]$ we have

$$
1=\sum_{H \leq G \text { up to conjugacy }} e_{H}
$$

where the $e_{H}$ are orthogonal primitive idempotents and each $e_{H}$ is a linear combination of elements $G / K$ with $K \leq H$. If $L$ contains no conjugate of $H$ then $e_{H} \cdot G / L=0$ and $e_{H} \downarrow_{L}^{G}=0$.

(ii) (Dress) Let $R$ denote the subring of $\mathbb{Q}$ in which all the prime divisors of $|G|$ are inverted except $p$. Then in the Burnside algebra $B(G)$ over $R$ we have

$$
1=\sum_{\substack{J \leq G \text { up to conjugacy } \\ J p \text {-perfect }}} f_{J}
$$

where the $f_{J}$ are primitive orthogonal idempotents in bijection with the conjugacy classes of p-perfect subgroups of $G$. For each p-perfect subgroup $J$ let $P \leq G$ be such that $J \triangleleft P$ and $P / J$ is a Sylow p-subgroup of $N_{G}(J) / J$. Then $f_{J}$ is a linear combination of elements $G / K$ with $K \leq P$. If $L$ contains no conjugate of $J$ then $f_{J} \cdot G / L=0$, and 
$f_{J} \downarrow_{L}^{G}=0$. In the Burnside ring over $\mathbb{Z}[1 /|G|]$ we have

$$
f_{J}=\sum_{\substack{J \leq K \leq P \\ K \text { up to conjugacy }}} e_{K} .
$$

We refer to [23, Lemma 3.4] for details. In fact the idempotents $e_{H}$ remain primitive in the Burnside algebra over a ring $R$ in which every prime divisor of $|G|$ is invertible, e.g., a field of characteristic 0 . Similarly the idempotents $f_{J}$ remain primitive in the Burnside algebra over a ring $R$ in which every prime divisor of $|G|$ is invertible except $p$, e.g., a field of characteristic $p$ or a local ring with residue field of characteristic $p$.

In studying the unions of blocks given by these Burnside idempotents, for each $p$-perfect subgroup $J$ of $G$ we introduce the notation $\operatorname{Mack}_{R}(G, J)$ to denote the full subcategory of $\operatorname{Mack}_{R}(G)$ whose objects are the Mackey functors $M$ for which $f_{J} \cdot M=M$. This definition works in situations where, for example, $R$ is a field of characteristic 0 or $p$, or a discrete valuation ring, because the $f_{J}$ are defined and so they act on Mackey functors over all of these coefficient rings. Our principal aim in the remainder of this section is to give various characterisations of the Mackey functors in $\operatorname{Mack}_{R}(G, J)$, in 9.5, 9.6, 9.7 and 9.14. We should mention that a theory closely related to 9.7 and 9.13 is developed by Oliver [16, Chapter 11] in the special case of subgroups $J$ which are cyclic of order prime to $p$.

Remark. All this analysis holds more generally for a set $\pi$ of prime divisors of $|G|$ rather than a single prime $p$. One has to consider a $\pi$-perfect subgroup $J$ and the corresponding idempotent $f_{J}$ of the Burnside algebra over a ring $R$ in which all prime divisors of $|G|$ are invertible except those in $\pi$. As we are mainly concerned with fields of characteristic 0 or $p$ (and their relationship appearing in the decomposition theory), we only develop the case of a single prime. The interested reader can easily modify the arguments.

(9.4) Lemma. Let $M$ be a Mackey functor and $L$ a subgroup of $G$ such that $M(H)=0$ for all subgroups $H$ of $L$. Then $G / L \cdot M=0$.

Proof. $(G / L \cdot M)(K)=\sum_{x \in[K \backslash G / L]} I_{K \cap{ }^{x} L}^{K} R_{K \cap{ }^{x} L}^{K} M(K)$. But these terms are images of $M\left(K \cap{ }^{x} L\right)=0$.

\section{(9.5) Theorem.}

(a) Let $R$ be a ring in which $|G|$ is invertible. Let $K$ be a subgroup of $G$ and let $M$ be an indecomposable Mackey functor over $R$. The following are equivalent:

(i) $e_{K} \cdot M=M$,

(ii) all subgroups $H$ minimal such that $M(H) \neq 0$ are conjugate to $K$,

(iii) $K$ is a minimal subgroup on which $M$ is non-zero.

(b) Let $R$ be a ring in which every prime divisor of $|G|$ is invertible, except for $p$ which is not invertible. Let $J$ be a p-perfect subgroup of $G$ and let $M$ be an indecomposable Mackey functor over $R$. The following are equivalent:

(i) $M \in \operatorname{Mack}_{R}(G, J)$, 
(ii) for all subgroups $H$ minimal such that $M(H) \neq 0, O^{p}(H) \sim_{G} J$,

(iii) there exists a subgroup $H$ minimal such that $M(H) \neq 0$, for which $O^{p}(H) \sim_{G} J$.

In particular it follows that for every Mackey functor $M \in \operatorname{Mack}_{R}(G, J)$, $M(H)=0$ unless $H \geq_{G} J$.

Proof. We prove only (b), the proof of (a) being obtained similarly by omitting references to $O^{p}$.

We show first that (i) $\Rightarrow$ (ii), so suppose that $M=f_{J} \cdot M$ and that $H$ is minimal such that $M(H) \neq 0$. We have $M(H)=f_{J} \downarrow_{H}^{G} \cdot M(H)$. But $f_{J} \downarrow_{H}^{G}=0$ unless $H \geq_{G} J$, so $O^{p}(H) \geq_{G} J$ since $J$ is $p$-perfect. On the other hand $f_{J}$ is a linear combination of terms $G / K$ with $K \leq P$ in the notation of 9.3, and so $f_{J} \downarrow_{H}^{G}$ is a linear combination of terms $H / K_{1}$ with $K_{1} \leq_{G} P$. If $O^{p}(H)>_{G} J$ then all these subgroups $K_{1}$ would be proper subgroups of $H$, and by minimality of $H$ we would deduce $f_{J} \downarrow_{H}^{G} \cdot M(H)=0$ by 9.4. From this contradiction we deduce that $O^{p}(H) \sim_{G} J$.

It is clear that (ii) $\Rightarrow$ (iii), and so it remains to prove that (iii) $\Rightarrow$ (i). But this follows also from the argument we have just given. In order to see this, suppose that $H$ is minimal such that $M(H) \neq 0$. Since $M$ is indecomposable, $M$ does lie in $\operatorname{Mack}\left(G, J_{1}\right)$ for some $p$-perfect subgroup $J_{1}$, and we have to show that $J_{1} \sim_{G} H$. But this was exactly the implication (i) $\Rightarrow$ (ii).

For the rest of this section, we specialize to a coefficient ring which is either a field $k$ or a complete discrete valuation ring $\mathscr{O}$. In 9.6 and 9.7 we give two further descriptions of the functors in $\operatorname{Mack}_{R}(G, J)$.

(9.6) Proposition. Let $S_{K, W}$ be a simple Mackey functor over a field $k$.

(i) If $|G|$ is invertible in $k$ then $e_{H} \cdot S_{K, W}=0$ unless $H$ and $K$ are conjugate, in which case $e_{K} \cdot S_{K, W}=S_{K, W}$. Thus the simple Mackey functors belonging to the blocks of Mackey functors determined by $e_{H}$ are precisely the $S_{H, W}$.

(ii) If $k$ is a field of characteristic $p$ then $f_{J} \cdot S_{K, W}=0$ unless $J$ and $O^{p}(K)$ are conjugate, in which case $f_{J} \cdot S_{K, W}=S_{K, W}$. Thus the simple Mackey functors in $\operatorname{Mack}_{k}(G, J)$ are precisely the $S_{K, W}$ with $J=$ $O^{p}(K)$. An arbitrary Mackey functor lies in $\operatorname{Mack}_{k}(G, J)$ precisely if all its composition factors do.

Proof. This is immediate from 9.5 since $K$ is (up to conjugacy) the unique subgroup $H$ of $G$ minimal such that $S_{K, W}(H) \neq 0$.

As an application of this (with $J=1$ ), the Mackey functors in $\operatorname{Mack}_{k}(G, 1)$ are precisely those which have all their compositions factors indexed by $p$ subgroups. Also if we have an indecomposable Mackey functor which has a composition factor $S_{H, V}$ where $H$ is a $p$-group, then all of its composition factors are $S_{K, W}$ where $K$ is a $p$-group. Thus, for example, if $H$ is a $p$ group then all composition factors of $P_{H, V}$ and of $\left(\operatorname{Inf}_{\bar{N}(H)}^{N(H)} F P_{V}\right) \uparrow_{N(H)}^{G}$ have minimum subgroups which are $p$-groups.

We now give a different characterisation of the Mackey functors lying in $\operatorname{Mack}_{R}(G, J)$ in terms of their vertices. Working over either a field or a complete discrete valuation ring, we recall from [17] that every indecomposable 
Mackey functor $M$ has up to conjugacy a unique minimal subgroup relative to which it is projective. This is called a vertex of $M$, denoted $\operatorname{vx}(M)$.

(9.7) Theorem. Assume that either $R=k$ is a field of characteristic $p$ or $R=\mathscr{O}$ is a complete discrete valuation ring with residue field of characteristic $p$, and let $J$ be a p-perfect subgroup of $G$. The indecomposable Mackey functors $M$ in $\operatorname{Mack}_{R}(G, J)$ are precisely those for which $O^{p}(\operatorname{vx} M)=J$.

The proof is based on the following lemma which will be also used in the next section.

(9.8) Lemma. Let $H$ be a subgroup of $G$ and $b \in B(H)$. Let $M$ be a Mackey functor for $G$. Let $m(b) \in \operatorname{End}_{M^{2 a c k}(H)}\left(M \downarrow_{H}^{G}\right)$ denote the multiplication by $b$, and similarly let $m\left(b \uparrow_{H}^{G}\right) \in \operatorname{End}_{\operatorname{Mack}_{R}(G)}(M)$ denote the multiplication by the element $b \uparrow_{H}^{G}$ of $B(G)$. Then

$$
m\left(b \uparrow_{H}^{G}\right)=\theta_{H} m(b) \uparrow_{H}^{G} \theta^{H},
$$

where $\theta^{H}: M \rightarrow M \downarrow_{H}^{G \uparrow} \uparrow_{H}^{G}$ and $\theta_{H}: M \downarrow_{H}^{G \uparrow} \uparrow_{H}^{G} \rightarrow M$ are the canonical morphisms. Proof. We can assume that $b$ is equal to a transitive $H$-set $H / K$. For every $G$-set $X$, the action of $H / K$ on $M \downarrow_{H}^{G}\left(X \downarrow_{H}^{G}\right)$ is equal to

$$
M \downarrow_{H}^{G}\left(X \downarrow_{H}^{G}\right) \stackrel{\left(M \downarrow_{H}^{G}\right)^{*}(\beta)}{\longrightarrow} M \downarrow_{H}^{G}\left(H \times_{K} X \downarrow_{H}^{G}\right) \stackrel{\left(M \downarrow_{H}^{G}\right)_{*}(\beta)}{\longrightarrow} M \downarrow_{H}^{G}\left(X \downarrow_{H}^{G}\right),
$$

where $\beta: H \times_{K} X \rightarrow X$ is the canonical map. Let also $\alpha: G \times_{H} X \rightarrow X$ be the analogous canonical map. Inducing to $G$ the composite above and composing with $\theta_{H}$ and $\theta^{H}$, we obtain

$$
\begin{aligned}
M(X) \stackrel{M^{*}(\alpha)}{\longrightarrow} M\left(G \times_{H} X\right) \stackrel{M^{*}(1 \times \beta)}{\longrightarrow} M\left(G \times_{H}\left(H \times_{K} X\right)\right) & \stackrel{M_{*}(1 \times \beta)}{\longrightarrow} M\left(G \times_{H} X\right) \stackrel{M_{*}(\alpha)}{\longrightarrow} M(X) .
\end{aligned}
$$

Identifying $G \times_{H}\left(H \times_{K} X\right)$ with $G \times_{K} X$ via the isomorphism

$$
G \times_{H}\left(H \times_{K} X\right) \rightarrow G \times_{K} X \quad(g,(h, x)) \mapsto(g h, x),
$$

the composite $\alpha \circ(1 \times \beta): G \times_{H}\left(H \times_{K} X\right) \rightarrow X$ is just equal to the natural map $G \times_{K} X \rightarrow X$ and so we have $M^{*}(1 \times \beta) M^{*}(\alpha)=\theta^{K}$ at $X$. Similarly we have $M_{*}(\alpha) M_{*}(1 \times \beta)=\theta_{K}$ at $X$ and this proves that the whole composite is equal to $\theta_{K} \theta^{K}$, which is the action of $G / K=(H / K) \uparrow_{H}^{G}$. This proves the result.

Proof of Theorem 9.7. Let $P$ be a subgroup such that $J \leq P \leq N_{G}(J)$ and $P / J$ is a Sylow $p$-subgroup of $N_{G}(J) / J$. We will show that $M$ is projective relative to $P$. This will be sufficient to prove the result, since by 9.5 it will follow that there is a vertex of $M$ with $J \leq \mathrm{vx} M \leq P$. The deduction we make from this is that not only do the Mackey functors in $\operatorname{Mack}_{R}(G, J)$ have the prescribed form, but if $M$ is any indecomposable Mackey functor, then the category $\operatorname{Mack}_{R}(G, J)$ in which it lies is determined by vx $M$ since $J=$ $O^{p}(\operatorname{vx} M)$.

To prove that $M$ is projective relative to $P$ we use Sasaki's criterion [17] that the identity endomorphism $1_{M}$ has an expression as a composite

$$
\left.M \stackrel{\theta^{P}}{\longrightarrow} M \downarrow_{P}^{G \uparrow_{P}^{G}} \stackrel{x \uparrow_{P}^{G}}{\longrightarrow} M \downarrow_{P}^{G_{\uparrow}^{G}}\right|_{P} ^{G} \stackrel{\theta_{P}}{\longrightarrow} M
$$


for some endomorphism $x \in \operatorname{End}_{\operatorname{Mack}_{R}(P)}\left(M \downarrow_{P}^{G}\right)$. Indeed this composite is equal to $I_{P}^{G}(x)$ in the Green functor $\mathscr{E}$ nd $(M)$ described by Sasaki (i.e., whose value at $H$ is $\left.\mathscr{E} \operatorname{nd}(M)(H)=\operatorname{End}_{\operatorname{Mack}_{R}(H)}\left(M \downarrow \downarrow_{H}^{G}\right)\right)$.

Since $f_{J} \cdot M=M, f_{J}$ acts as the identity on $M$, and by $9.3 f_{J}$ lies in the span of $G$-sets $G / K$ with $K \leq P$. Thus we can write $f_{J}=\sum \lambda_{K} G / K \in B(G)$ for such $K$ and coefficients $\lambda_{K}$. If we set $x=\sum \lambda_{K} P / K \in B(P)$, then $x \uparrow_{P}^{G}=$ $f_{J}$ and so by Lemma 9.8 ,

$$
\mathrm{id}=m\left(f_{J}\right)=\theta_{P} m(x) \uparrow_{P}^{G} \theta^{P} \in \operatorname{End}_{\operatorname{Mack}_{R}(G)}(M) .
$$

This completes the proof of the theorem.

The following fact becomes easy in the light of 9.6 and 9.7.

(9.9) Corollary. Let $M$ be an indecomposable Mackey functor over a field $k$ of characteristic $p$, and let $S_{H, V}$ be any composition factor of $M$. Then $O^{p}(H)=$ $O^{p}(\operatorname{vx} M)$.

For example, if $M$ is indecomposable and $M(H) \neq 0$ for some $p$-subgroup $H$, then $\mathrm{vx} M$ is a $p$-group.

We now apply Proposition 9.6 to the computation of the decomposition matrix. The effect of this division into a union of blocks is that in Proposition 7.3 we need only consider at one time the $S_{H, V}$ where $O^{p}(H)$ is a fixed subgroup. To formalize this, suppose that $R=\mathscr{O}$ is a complete discrete valuation ring with field of fractions $K$ of characteristic 0 and residue field $k$ of characteristic $p$.

(9.10) Corollary. Let $J$ be a p-perfect subgroup of $G$. Then the square in Proposition 7.3 gives rise to a commutative square

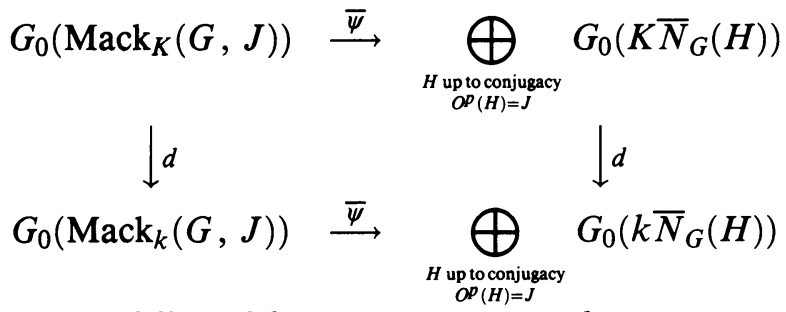

where $\bar{\psi}$ denotes $\psi$ followed by projection onto those summands which have $O^{p}(H)=J$. The maps $\bar{\psi}$ are isomorphisms and the maps $d$ are surjections.

Proof. The argument is the same as was used in 6.1 and 7.3. The new aspects are that decomposition of Mackey functors does indeed send $\operatorname{Mack}_{K}(G, J)$ to $\operatorname{Mack}_{k}(G, J)$, since these are the unions of blocks in characteristic 0 and $p$ determined by the idempotent $f_{J}$, and also that the new maps $\bar{\psi}$ are isomorphisms. This is because the domain and codomain of $\bar{\psi}$ have bases indexed by pairs $(H, V)$ subject to the restriction that $O^{p}(H)=J$ and the matrix of $\bar{\psi}$ is just the square submatrix of $\psi$ consisting of the entries which correspond to these new basis elements. Since the matrix of $\psi$ was triangular with 1's down the diagonal, the matrix of $\bar{\psi}$ has this property also.

We present two more corollaries of Proposition 9.6. It will help to notice that in any finite group $G$ there is a bijection between conjugacy classes of $p$-perfect subgroups $J$ and conjugacy classes of subgroups $H$ such that $p \nmid\left|N_{G}(H): H\right|$, given by $J=O^{p}(H)$ and $H / J \in \operatorname{Syl}_{p}\left(N_{G}(J) / J\right)$. 


\section{(9.11) Corollary.}

(i) If $k$ is a field in which $|G|$ is invertible we have $S_{H, k}=e_{H} \cdot B^{G}$ for every subgroup $H$.

(ii) If $k$ is a field of characteristic $p$ we have $P_{H, k}=f_{J} \cdot B^{G}$ for each subgroup $H$ with $p \nmid\left|N_{G}(H): H\right|$, where $J=O^{p}(H)$.

Proof. (i) The indecomposable Mackey functor summands of $B^{G}$ are precisely the functors $e_{K} \cdot B^{G}$ by 8.2(ii) and 9.3 , and also by 8.9 they have the form $P_{H, k}$, which equals $S_{H, k}$ in this case. The idempotent which gives $S_{H, k}$ must be $e_{H}$ since by 9.6 this is the only one which acts in a non-zero fashion on $S_{H, k}$.

(ii) The proof is similar to (i) but now the indecomposable summands of $B^{G}$ are the $f_{J} \cdot B^{G}$ by 8.2 and 9.3 , and also they have the form $P_{H, k}$ with $p \nmid\left|N_{G}(H): H\right|$ by 8.9. The idempotent which gives $P_{H, k}$ must be $f_{J}$ since by 9.6 this is the only one which acts in a non-zero fashion on the top composition factor $S_{H, k}$ of $P_{H, k}$.

(9.12) Corollary. Let $H$ be a subgroup of $G$ for which $p \nmid\left|N_{G}(H): H\right|$ and let $\mathscr{O}$ be a complete discrete valuation ring with residue field $k$ of characteristic $p$ and quotient field $K$ of characteristic 0 . Then the decomposition number $d_{S_{L, V}, S_{H, k}}$ satisfies

$$
d_{S_{L, V}, S_{H, k}}= \begin{cases}1 & \text { if } O^{p}(L) \sim_{G} O^{p}(H) \text { and } V=K, \\ 0 & \text { otherwise. }\end{cases}
$$

Proof. By $9.11 P_{H, k}=f_{J} \cdot B^{G}$ where $J=O^{p}(H)$, and the lifting of this to $\mathscr{O}$ is $\hat{P}_{H, k}=f_{J} \cdot B^{G}$ where now the Burnside functor is taken over $\mathscr{O}$. By 7.1(ii) the decomposition number is the multiplicity of $S_{L, V}$ in $K \otimes_{R} \hat{P}_{H, k}$ which is

$$
\bigoplus_{\substack{J \leq x \leq H \\ x \text { up ioconjugacy }}} e_{X} \cdot B^{G} \cong \bigoplus_{\substack{\leq \leq x \leq H \\ x \text { uploconjugacy }}} S_{X, K}
$$

using 9.3(ii) and 9.11 again. Thus $S_{L, V}$ occurs just once if we have $V=K$ and $O^{p}(L) \sim_{G} J$, and does not occur otherwise.

Given some naturally occurring Mackey functor it is useful to know how one may break it apart into the summands given by Burnside idempotents. We conclude by showing how our approach here leads to a concrete identification of these summands. Let $M$ be a Mackey functor and let $J$ be a $p$-perfect subgroup of $G$. We define as a subfunctor of $M$

$$
M^{J}=\left\langle M(X) \mid O^{p}(X) \leq_{G} J\right\rangle .
$$

Recall that by Proposition 2.4, we have

$$
M^{J}(H)=\sum_{\substack{O^{\phi}(X) \leq G J \\ X \leq H}} I_{X}^{H}(M(X)) .
$$

(9.13) Theorem. Assume that either $R=k$ is a field of characteristic $p$ or $R=\mathscr{O}$ is a complete discrete valuation ring with residue field of characteristic $p$, let $J$ be a p-perfect subgroup of $G$ and let $M$ be a Mackey functor over $R$. For every p-perfect subgroup $J$ of $G, M^{J}$ is a direct summand of $M$. Furthermore,

$$
f_{J} \cdot M \cong M^{J} / \sum_{p \text {-perfect } L<J} M^{L} .
$$


Proof. Let us write

$$
N=\bigoplus_{\substack{p \text {-perfect } L \leq J \\ L \text { up to conjugacy }}} f_{L} \cdot M,
$$

as a subfunctor of $M$. We show that $M^{J}=N$ from which the result is immediate. Firstly we observe that $N$ is projective relative to the subgroups $X$ with $O^{p}(X) \leq_{G} J$, by 9.7. It is well-known [9] that this implies that

$$
N(H)=\sum_{\substack{O^{p}(X) \leq \leq_{G} J \\ X \leq H}} I_{X}^{H}(N(X)) .
$$

This follows for instance from the fact that $I_{X}^{H}$ is surjective for all $H \geq X$ in a functor induced from $X$, so that the same holds for any direct summand of such a functor. Thus $N=\left\langle N(X) \mid O^{p}(X) \leq_{G} J\right\rangle \subseteq M^{J}$. On the other hand

$$
M=\bigoplus_{\substack{p \text {-perfect } L \\ L \text { up to conjugacy }}} f_{L} \cdot M,
$$

and if $O^{p}(X) \leq_{G} J$ but $L \underline{Z}_{G} J$ then $\left(f_{L} \cdot M\right)(X)=0$ by 9.5 applied to $f_{L} \cdot M$. Thus $M(X) \subseteq N(X)$ and so $M^{J} \subseteq N$. We conclude that $N=M^{J}$.

Remark. In case $M$ is a Mackey functor over a field in which $|G|$ is invertible there is a similar description of $e_{H} \cdot M$ as

$$
\left\langle M(X) \mid X \leq_{G} H\right\rangle /\left\langle M(X) \mid X<_{G} H\right\rangle .
$$

We leave it to the reader to make the necessary changes and simplifications.

(9.14) Corollary. With the assumptions of 9.13, $M$ lies in $\operatorname{Mack}_{R}(G, J)$ if and only if

(i) $M(H)=0$ unless $H \geq_{G} J$, and

(ii) $M=\left\langle M(X) \mid O^{p}(X) \sim_{G} J\right\rangle$.

10. AN EQUIVALENCE OF CATEGORIES: THE REDUCTION TO $p$-SUBGROUPS

In this section, we prove a result which reduces questions about arbitrary Mackey functors to ones associated with $p$-groups. Explicitly let $J$ be a $p$ perfect subgroup of $G$ and let $N=N_{G}(J), \bar{N}=N / J$. We work over a base ring $R$ in which every prime divisor of $|G|$ different from $p$ is invertible. Thus the primitive idempotent $f_{J}$ of the Burnside algebra $B(G)$ over $R$ is defined (see Theorem 9.3). In order to emphasize the dependence on $G$, we write $f_{J}^{G}=f_{J}$. Recall that $\operatorname{Mack}_{R}(G, J)$ denotes the full subcategory of $\operatorname{Mack}_{R}(G)$ whose objects are the Mackey functors $M$ such that $f_{J}^{G} \cdot M=M$. Similarly we consider $\operatorname{Mack}_{R}(N, J)$ and $\operatorname{Mack}_{R}(\bar{N}, 1)$. We are going to see that inflation $\operatorname{Inf} \frac{N}{N}$ maps $\operatorname{Mack}_{R}(\bar{N}, 1)$ into $\operatorname{Mack}_{R}(N, J)$ and that induction $\uparrow_{N}^{G}$ maps $\operatorname{Mack}_{R}(N, J)$ into $\operatorname{Mack}_{R}(G, J)$. Our main result is the following theorem. A related result is Theorem $C$ of Yoshida's paper [25].

(10.1) Theorem. The categories $\operatorname{Mack}_{R}(\bar{N}, 1)$ and $\operatorname{Mack}_{R}(G, J)$ are equivalent. More precisely:

(i) The functor $\operatorname{Inf} \frac{N}{N}: \operatorname{Mack}_{R}(\bar{N}, 1) \rightarrow \operatorname{Mack}_{R}(N, J)$ is an isomorphism of categories. 
(ii) The functor $\uparrow_{N}^{G}$ : $\operatorname{Mack}_{R}(N, J) \rightarrow \operatorname{Mack}_{R}(G, J)$ is an equivalence of categories, whose inverse is the restriction functor followed by multiplication by the idempotent $f_{J}^{N}$.

(10.2) Remark. The theorem also holds for an arbitrary set of primes $\pi$ rather than a single prime $p$. One has to work over a ring $R$ in which every prime divisor of $|G|$ outside $\pi$ is invertible. One takes a $\pi$-perfect subgroup $J$ and the corresponding primitive idempotent $f_{J}^{G}$ of $B(G)$, as explained in [23]. The proof below applies without change.

Proof of Theorem 10.1. The proof of the theorem is based on a detailed analysis of the idempotents $f_{J}^{G}$ and $f_{J}^{N}$, and of their action on Mackey functors. We proceed in a series of lemmas. The first two deal with part (i).

(10.3) Lemma. Let $\phi: B(N) \rightarrow B(\bar{N})$ be the ring homomorphism which maps an $N$-set $X$ to the $\bar{N}$-set $X^{J}$ of $J$-fixed points in $X$.

(i) If $S \leq N$, then $\phi(N / S)=\bar{N} / \bar{S}$ if $J \leq S$ and $\phi(N / S)=0$ if $J \mathbb{S}$.

(ii) $\phi\left(f_{J}^{N}\right)=f_{1}^{\bar{N}}$.

Proof. (i) This is straightforward.

(ii) Since the Burnside algebra over a localization of $\mathbb{Z}$ embeds into the Burnside algebra over $\mathbb{Q}$, we can work over $\mathbb{Q}$. If $J \leq S \leq N$, consider the ring homomorphism $\phi_{S}: B(N) \rightarrow \mathbb{Q}$ which maps an $N$-set $X$ to the number $\left|X^{S}\right|$ of $S$-fixed points in $X$. Clearly $\phi_{S}$ factorizes as $\phi_{S}=\phi_{\bar{S}} \phi$. The primitive idempotent $e_{K}^{N}$ of $B(N)$ satisfies by definition $\phi_{S}\left(e_{K}^{N}\right)=1$ if $K$ is $N$-conjugate to $S$ and $\phi_{S}\left(e_{K}^{N}\right)=0$ if $K$ is not $N$-conjugate to $S$. Thus $f_{J}^{N}$ is characterized by the property $\phi_{S}\left(f_{J}^{N}\right)=1$ if and only if $O^{p}(S)=J$. It follows that $\phi\left(f_{J}^{N}\right)$ is characterized by the property $\phi_{\bar{S}}\left(\phi\left(f_{J}^{N}\right)\right)=1$ if and only if $O^{p}(\bar{S})=1$, and this means that $\phi\left(f_{J}^{N}\right)=f_{1}^{\bar{N}}$.

\section{(10.4) Lemma.}

(i) Let $\bar{L}$ be a Mackey functor in $\operatorname{Mack}_{R}(\bar{N}, 1)$. Then the Mackey functor $L=\operatorname{Inf} \frac{N}{N}(\bar{L})$ lies in $\operatorname{Mack}_{R}(N, J)$.

(ii) Let $L$ be a Mackey functor in $\operatorname{Mack}_{R}(N, J)$. Then $L=\operatorname{Inf} \frac{N}{N}(\bar{L})$ for some (unique) Mackey functor $\bar{L}$, and $\bar{L}$ lies in $\operatorname{Mack}_{R}(\bar{N}, 1)$.

Proof. Write $f_{J}^{N}=f^{\prime}+f^{\prime \prime}$ where $f^{\prime}$ lies in the $R$-linear span of the transitive $N$-sets $N / S$ with $S \geq J$ and $f^{\prime \prime}$ lies in the $R$-linear span of the transitive $N$-sets $N / S$ with $S \nsupseteq J$. Then by Lemma 10.3, we have $\phi\left(f^{\prime \prime}\right)=0$ and $\phi\left(f^{\prime}\right)=f_{1}^{\bar{N}}$.

(i) The action of $N / S$ on $L(K)$ is equal to $\sum_{g \in[K \backslash N / S]} I_{K \cap 8 S}^{K} R_{K \cap 8 S}^{K}$. Since $L$ is inflated, it vanishes on subgroups not containing $J$ and the action of $N / S$ is zero if $S \geq J$. If both $K$ and $S$ contain $J$, then all subgroups involved contain $J$, so that the action of $N / S$ is obtained from the action of $\bar{N} / \bar{S}$ on $\bar{L}$. It follows that $f^{\prime \prime}$ acts by zero and that the action of $f^{\prime}$ is obtained from that of $\phi\left(f^{\prime}\right)=f_{1}^{\bar{N}}$ which is the identity by assumption. Therefore $f_{J}^{N}$ acts as the identity on $L$, as required.

(ii) By Theorem 9.5, $L$ vanishes on subgroups not containing $J$ (because $J$ is a normal subgroup of $N$ ). Therefore $L$ is obtained by inflation from a 
unique Mackey functor $\bar{L}$ for $\bar{N}$. Since $L$ vanishes on subgroups not containing $J$, the argument above shows that $f^{\prime \prime}$ acts by zero and so the action of $f^{\prime}$ is equal to the action of $f_{J}^{N}$ which is the identity. But since the action of $f^{\prime}$ on $L$ corresponds to that of $f_{1}^{\bar{N}}=\phi\left(f^{\prime}\right)$ on $\bar{L}$, we see that $f_{1}^{\bar{N}}$ acts as the identity on $\bar{L}$.

It is clear that Lemma 10.4 immediately implies part (i) of the theorem. The rest of this section is devoted to the proof of part (ii).

(10.5) Lemma.

(i) Let $K_{1}$ and $K_{2}$ be subgroups such that $J \leq K_{i} \leq N$ and $K_{i} / J$ is a p-group. If $K_{2}={ }^{g} K_{1}$ for some $g \in G$, then $g \in N$.

(ii) In $B(G)$, we have $f_{J}^{N} \uparrow_{N}^{G}=f_{J}^{G}$.

(iii) In $B(N)$, we have

$$
f_{J}^{G} \downarrow_{N}^{G}=\sum_{g \in\left[N \backslash T_{G}(J, N) / N\right]} f_{8 J}^{N},
$$

where

$$
T_{G}(J, N)=\left\{\left.g \in G\right|^{g} J \leq N\right\} .
$$

(iv) In $B\left(N \cap{ }^{g} N\right)$, we have

$$
f_{J}^{N} \downarrow_{N \cap B_{N}}^{N}= \begin{cases}f_{J}^{N \cap \cap^{8} N} & \text { if } J \leq s^{g} N\left(\text { i.e., } g^{-1} \in T_{G}(J, N)\right), \\ 0 & \text { otherwise. }\end{cases}
$$

Proof. (i) We have $J=O^{p}\left(K_{2}\right)$ and $J=O^{p}\left(K_{1}\right)$, so that ${ }^{g} J=O^{p}\left({ }^{g} K_{1}\right)$. Thus $J={ }^{g} J$, that is, $g \in N$.

(ii) Since the Burnside algebra over a localization of $\mathbb{Z}$ embeds into the Burnside algebra over $\mathbb{Q}$, we can work over $\mathbb{Q}$. By Theorem 9.3, we know that

$$
f_{J}^{N}=\sum_{\substack{J \leq K \leq P \\ K \text { upio } N \text {-conjugacy }}} e_{K}^{N},
$$

where $P / J$ is a Sylow $p$-subgroup of $N / J$ and $e_{K}^{N}$ denotes the primitive idempotent of $B(N)$ corresponding to the subgroup $K$. Moreover it is wellknown (see $[23,3.5])$ that one has

$$
e_{K}^{N} \uparrow_{N}^{G}=\left|N_{G}(K): N_{N}(K)\right| \cdot e_{K}^{G} .
$$

But $N_{G}(K) \leq N_{G}\left(O^{p}(K)\right)=N_{G}(J)=N$ so that $N_{N}(K)=N_{G}(K)$. Therefore

$$
f_{J}^{N} \uparrow_{N}^{G}=\sum_{\substack{J \leq K \leq P \\ \kappa \text { upto } N \text {-conjugacy }}} e_{K}^{G} .
$$

By the first part of this lemma, we know that $N$-conjugacy of such subgroups $K$ coincides with $G$-conjugacy. Therefore we obtain the expression of $f_{J}^{G}$ given by Theorem 9.3 , as required.

(iii) We use again the expression of $f_{J}^{G}$ as a sum of primitive idempotents $e_{K}^{G}$ in the Burnside algebra over $\mathbb{Q}$. We have $e_{K}^{G} \downarrow_{N}^{G}=\sum_{i} e_{K_{i}}^{N}$ where the subgroups $K_{i}$ are representatives of the $N$-conjugacy classes of subgroups of $N$ which are conjugate to $K$ in $G$. (Note that we are not in the situation of (i), because $K_{i}$ 
need not even contain $J$.) Now grouping together all subgroups with the same $O^{p}$ (which is necessarily a $G$-conjugate of $J$ contained in $N$ ), we obtain

$$
\begin{aligned}
f_{J}^{G} \downarrow_{N}^{G} & =\sum_{\substack{g \in[N \backslash G / N] \\
g J \leq N}} \sum_{\substack{g_{J} \leq K \leq N, o^{p}(K)=g_{J} \\
K \text { upto } N \text {-conjugacy }}} e_{K}^{N} \\
& =\sum_{g \in\left[N \backslash T_{G}(J, N) / N\right]} f_{g_{J}}^{N} .
\end{aligned}
$$

Note that the sum is over $(N, N)$-double cosets because we are considering the set of $G$-conjugates of $J$ (in bijection with $G / N$ ) and taking only representatives of $N$-conjugacy classes.

(iv) Once again, we work in the Burnside algebra over $\mathbb{Q}$. If $J \varangle^{g} N$ and $J \leq K \leq N$, we have $K \not N \cap{ }^{g} N$ and so $e_{K}^{N} \downarrow_{N \cap 8 N}^{N}=0$. It follows that $f_{J}^{N} \downarrow_{N \cap 8 N}^{N}=0$. Assume now that $J \leq{ }^{g} N$. Then since $J$ is a normal subgroup of $N \cap{ }^{g} N$, we have

$$
\begin{aligned}
& f_{J}^{N} \downarrow_{N \cap 8 N}^{N}=\sum_{\substack{j \leq K \leq N, o^{p}(K)=J \\
K \text { upto } N \text {-conjugacy }}} e_{K}^{N} \downarrow_{N \cap 8 N}^{N}=\sum_{\substack{J \leq K \leq N \cap g_{N}, o^{p}(K)=J \\
K \text { up to } N \text {-conjugacy }}} \sum_{i} e_{K_{i}}^{N \cap g^{g} N} \\
& =\sum_{\substack{J \leq L \leq N \cap g_{N}, o_{(L)=J} \\
L \text { upto }\left(N \cap 8_{N}\right) \text {-conjugacy }}} e_{L}^{N \cap g^{8} N}=f_{J}^{N \cap{ }^{8} N},
\end{aligned}
$$

where the inner sum runs over representatives $K_{i}$ of the $\left(N \cap{ }^{g} N\right)$-conjugacy classes of subgroups which are conjugate to $K$ in $N$.

(10.6) Lemma. Let $H$ be a subgroup of $G$ and $g \in G$. Let $b \in B(G)$.

(i) Let $L$ be a Mackey functor for $H$. Under the identification $L_{\uparrow}^{G}(X)=$ $L\left(X \downarrow_{H}^{G}\right)$, the action of $b$ on $L \uparrow_{H}^{G}(X)$ is equal to the action of $b \downarrow_{H}^{G}$ on $L\left(X \downarrow_{H}^{G}\right)$. In particular if $b \downarrow_{H}^{G}$ acts as the identity on $L$, then $b$ acts as the identity on $L \uparrow_{H}^{G}$.

(ii) Let $M$ be a Mackey functor for $G$. Under the identification $M_{\downarrow_{H}}^{G}(Y)=$ $M\left(Y \uparrow_{H}^{G}\right)$, the action of $b \downarrow_{H}^{G}$ on $M \downarrow_{H}^{G}(Y)$ is equal to the action of $b$ on $M\left(Y \uparrow_{H}^{G}\right)$. In particular if $b$ acts as the identity on $M$, then $b \downarrow_{H}^{G}$ acts as the identity on $M \downarrow_{H}^{G}$.

(iii) Let $L$ be a Mackey functor for $H$ and $a \in B(H)$. Under the identification ${ }^{g} L(Y)=L\left({ }^{-1} Y\right)$, the action of $g_{a}$ on ${ }^{g} L(Y)$ is equal to the action of $a$ on $L\left(g^{-1} Y\right)$. In particular if a acts as the identity on $L$, then $g_{a}$ acts as the identity on ${ }^{g} L$.

Proof. (i) By linearity, we can assume that $b$ is equal to some $G$-set $Z$. The action of $Z$ on $L \uparrow_{H}^{G}(X)$ is the composite

$$
L \uparrow_{H}^{G}(X) \stackrel{\left(L \uparrow_{H}^{G}\right)^{*}\left(\mathrm{pr}_{2}\right)}{\longrightarrow} L \uparrow_{H}^{G}(Z \times X) \stackrel{\left(L \uparrow_{H}^{G}\right)_{*}\left(\mathrm{pr}_{2}\right)}{\longrightarrow} L \uparrow_{H}^{G}(X),
$$

which, by definition of induction, is equal to

$$
L\left(X \downarrow_{H}^{G}\right) \stackrel{L^{*}\left(\mathrm{pr}_{2} \downarrow_{H}^{G}\right)}{\longrightarrow} L\left((Z \times X) \downarrow_{H}^{G}\right) \stackrel{L_{*}\left(\mathrm{pr}_{2} \downarrow_{H}^{G}\right)}{\longrightarrow} L\left(X \downarrow_{H}^{G}\right) .
$$

This is the action of $Z \downarrow_{H}^{G}$, as required. 
(ii) Again we can assume that $b$ is equal to a $G$-set $Z$. The action of $Z \downarrow_{H}^{G}$ on $M \downarrow_{H}^{G}(Y)$ is the composite

$$
M \downarrow_{H}^{G}(Y) \stackrel{\left(M \downarrow_{H}^{G}\right)^{*}\left(\mathrm{pr}_{2}\right)}{\longrightarrow} M \downarrow_{H}^{G}\left(Z \downarrow_{H}^{G} \times Y\right) \stackrel{\left(M \downarrow_{H}^{G}\right) *\left(\mathrm{pr}_{2}\right)}{\longrightarrow} M \downarrow_{H}^{G}(Y),
$$

which, by definition of restriction, is equal to

$$
M\left(Y \uparrow_{H}^{G}\right) \stackrel{M^{*}\left(\mathrm{pr}_{2}{ }_{H}^{G}\right)}{\longrightarrow} M\left(\left(Z \downarrow_{H}^{G} \times Y\right) \uparrow_{H}^{G}\right) \stackrel{M_{*}\left(\mathrm{pr}_{2} \uparrow_{H}^{G}\right)}{\longrightarrow} M\left(Y \uparrow_{H}^{G}\right) .
$$

Now there is a canonical isomorphism of $G$-sets $\left(Z \downarrow_{H}^{G} \times Y\right) \uparrow_{H}^{G} \cong Z \times Y \uparrow_{H}^{G}$, and the map $\operatorname{pr}_{2} \uparrow_{H}^{G}$ corresponds simply to the second projection $\mathrm{pr}_{2}$. Therefore the composite above is equal to

$$
M\left(Y \uparrow_{H}^{G}\right) \stackrel{M^{*}\left(\mathrm{pr}_{2}\right)}{\longrightarrow} M\left(Z \times Y \uparrow_{H}^{G}\right) \stackrel{M_{*}\left(\mathrm{pr}_{2}\right)}{\longrightarrow} M\left(Y \uparrow_{H}^{G}\right),
$$

which is the action of $Z$, as required.

(iii) The proof is straightforward.

Combining Lemma 10.6 with Lemma 10.5, we obtain the following result.

(10.7) Lemma.

(i) Let $L$ be a Mackey functor lying in $\operatorname{Mack}_{R}(N, J)$. Then $L \uparrow_{N}^{G}$ lies in $\operatorname{Mack}_{R}(G, J)$.

(ii) Let $M$ be a Mackey functor lying in $\operatorname{Mack}_{R}(G, J)$. Then $M \downarrow_{N}^{G}$ can be decomposed as a direct sum $M \downarrow_{N}^{G}=\bigoplus_{g \in\left[N \backslash T_{G}(J, N) / N\right]} M_{g}$ where $M_{g}$ is a Mackey functor in $\operatorname{Mack}_{R}\left(N,{ }^{g}\right)$.

(iii) Let $L$ be a Mackey functor lying in $\operatorname{Mack}_{R}(N, J)$. Then ${ }^{g} L$ lies in $\operatorname{Mack}_{R}\left({ }^{g} N,{ }^{g} J\right)$.

(iv) Let $L$ be a Mackey functor lying in $\operatorname{Mack}_{R}(N, J)$. If $g^{-1} \notin$ $T_{G}(J, N)$, then $L \downarrow_{N \cap 8 N}^{N}=0$, while $L \downarrow_{N \cap B_{N}}^{N}$ lies in $\operatorname{Mack}_{R}\left(N \cap{ }^{g} N, J\right)$ if $g^{-1} \in T_{G}(J, N)$.

Proof. (i) Since $f_{J}^{N}$ acts on $L$ as the identity, $f_{J}^{G} \downarrow_{N}^{G}=\sum_{g \in\left[N \backslash T_{G}(J, N) / N\right]} f_{8 J}^{N}$ also acts on $L$ as the identity (because for $g \notin N$, the idempotent $f_{8 J}^{N}$ is orthogonal to $f_{J}^{N}$, hence acts by zero). By Lemma 10.6 (i), this implies that $f_{J}^{G}$ acts as the identity on $L \uparrow_{N}^{G}$.

(ii) Since $f_{J}^{G}$ acts on $M$ as the identity, $f_{J}^{G} \downarrow_{N}^{G}=\sum_{g \in\left[N \backslash T_{G}(J, N) / N\right]} f_{8 J}^{N}$ acts as the identity on $M \downarrow_{N}^{G}$, by Lemma 10.6 (ii). The result follows by setting $M_{g}=f_{g J}^{N} \cdot M \downarrow_{N}^{G}$.

(iii) This is straightforward by Lemma 10.6 (iii).

(iv) This is immediate by Lemma 10.6 (ii) and Lemma 10.5 (iv).

(10.8) Remark. Part (i) holds more generally for an arbitrary subgroup $H$ of $G$ containing $J$ : if $L$ lies in $\operatorname{Mack}_{R}(H, J)$, then $L \uparrow_{H}^{G}$ lies in $\operatorname{Mack}_{R}(G, J)$. The proof is similar and is based on the observation that $f_{J}^{H}$ appears necessarily as an orthogonal summand of the idempotent $f_{J}^{G} \downarrow_{H}^{G}$.

We know now that the induction functor maps $\operatorname{Mack}_{R}(N, J)$ into $\operatorname{Mack}_{R}(G, J)$ and we have to show that this is an equivalence. Let us denote this induction functor by

$$
\mathscr{I}: \operatorname{Mack}_{R}(N, J) \rightarrow \operatorname{Mack}_{R}(G, J) .
$$


Now consider the functor

$$
\mathscr{R}: \operatorname{Mack}_{R}(G, J) \rightarrow \operatorname{Mack}_{R}(N, J),
$$

defined as the composite of the restriction functor and the multiplication by the idempotent $f_{J}^{N}$. In the notation of Lemma 10.7 (ii), we have $\mathscr{R}(M)=M_{1}$. As the induction and restriction functors are adjoint on both sides, we also have that $\mathscr{R}$ is both the left and the right adjoint of $\mathscr{I}$. Indeed, for $L$ in $\operatorname{Mack}_{R}(N, J)$, any morphism $L \rightarrow M \downarrow_{N}^{G}$ factorizes necessarily through $f_{J}^{N} \cdot M \downarrow_{N}^{G}=\mathscr{R}(M)$, and similarly for a morphism $M \downarrow_{N}^{G} \rightarrow L$ (because any morphism commutes with the action of the central idempotent $f_{J}^{N}$ ). We shall prove the desired equivalence by showing that the units and counits of the adjunctions are isomorphisms. Thus we need to describe them explicitly.

For a $G$-set $X$, let

$$
p_{X}: X \downarrow_{N}^{G}{ }_{N}^{G} \rightarrow X, \quad(g, x) \mapsto g x
$$

be the canonical map. (Via the identification $X \downarrow_{N}^{G} \uparrow_{N}^{G} \cong G / N \times X$, the map $p_{X}$ is just the second projection.) For an $N$-set $Y$, let

$$
i_{Y}: Y \rightarrow Y \uparrow_{N}^{G} \downarrow_{N}^{G}, \quad y \mapsto(1, y)
$$

be the canonical map. Let $M$ be a Mackey functor for $G$ and $L$ a Mackey functor for $N$. From Proposition 4.2 in [20], we know that the units and counits for the adjunctions between induction and restriction are the following morphisms:

$$
\begin{aligned}
M_{*}\left(p_{X}\right) & : M \downarrow_{N}^{G} \uparrow_{N}^{G}(X)=M\left(X \downarrow_{N}^{G} \uparrow_{N}^{G}\right) \longrightarrow M(X), \\
L_{*}\left(i_{Y}\right) & : L(Y) \longrightarrow L \uparrow_{N}^{G} \downarrow_{N}^{G}(Y)=L\left(Y \uparrow_{N}^{G} \downarrow_{N}^{G}\right), \\
L^{*}\left(i_{Y}\right) & : L \uparrow_{N}^{G} \downarrow_{N}^{G}(Y)=L\left(Y \uparrow_{N}^{G} \downarrow_{N}^{G}\right) \longrightarrow L(Y), \\
M^{*}\left(p_{X}\right) & : M(X) \longrightarrow M \downarrow_{N}^{G}{ }_{N}^{G}(X)=M\left(X \downarrow_{N}^{G} \uparrow_{N}^{G}\right) .
\end{aligned}
$$

The units and counits of the adjunctions between $\mathscr{R}$ and $\mathscr{J}$ are obtained from the units and counits above by composing them either with one of the inclusions

$$
j\left(M \downarrow_{N}^{G}\right): \mathscr{R}(M) \rightarrow M \downarrow_{N}^{G}, \quad j\left(L \uparrow_{N}^{G} \downarrow_{N}^{G}\right): \mathscr{R}\left(L \uparrow_{N}^{G}\right) \rightarrow L \uparrow_{N}^{G} \downarrow_{N}^{G},
$$

or with one of the projections

$$
m\left(f_{J}^{N}\right): M \downarrow_{N}^{G} \rightarrow \mathscr{R}(M), \quad m\left(f_{J}^{N}\right): L \uparrow_{N}^{G} \downarrow_{N}^{G} \rightarrow \mathscr{R}\left(L \uparrow_{N}^{G}\right),
$$

where $m\left(f_{J}^{N}\right)$ denotes the multiplication by $f_{J}^{N}$. Therefore we obtain the following four morphisms:

$$
\begin{aligned}
& \epsilon_{M}: \mathscr{\mathscr { R }}(M) \stackrel{j\left(M_{\downarrow_{N}}^{G} \uparrow_{N}^{G}\right.}{\longrightarrow} M \downarrow_{N}^{G} \uparrow_{N}^{G} \stackrel{M_{*}(p)}{\longrightarrow} M, \\
& \sigma_{L}: L \stackrel{L_{*}(i)}{\longrightarrow} L \uparrow_{N}^{G} \downarrow_{N}^{G} \stackrel{m\left(f_{J}^{N}\right)}{\longrightarrow} \mathscr{R} \mathscr{J}(L), \\
& \pi_{L}: \mathscr{R} \mathscr{J}(L) \stackrel{j\left(L \uparrow_{N}^{G} \downarrow_{N}^{G}\right)}{\longrightarrow} L \uparrow_{N}^{G} \downarrow_{N}^{G} \stackrel{L^{*}(i)}{\longrightarrow} L, \\
& \eta_{M}: M \stackrel{M^{*}(p)}{\longrightarrow} M \downarrow_{N}^{G} \uparrow_{N}^{G} \stackrel{m\left(f_{J}^{N} \uparrow_{N}^{G}\right.}{\longrightarrow} \mathscr{I R}(M) .
\end{aligned}
$$

Of course $M^{*}(p)$ denotes the morphism which, on evaluation on a $G$-set $X$, is equal to the map $M^{*}\left(p_{X}\right)$ described above (and similarly with $M_{*}(p), L^{*}(i)$ and $\left.L_{*}(i)\right)$.

The following lemma summarizes our discussion. 
(10.9) Lemma. The functor

$$
\mathscr{R}: \operatorname{Mack}_{R}(G, J) \rightarrow \operatorname{Mack}_{R}(N, J)
$$

is both the right and the left adjoint of the induction functor

$$
\mathscr{I}: \operatorname{Mack}_{R}(N, J) \rightarrow \operatorname{Mack}_{R}(G, J) .
$$

The units and counits of the adjunctions are given by the four morphisms $\epsilon_{M}$, $\sigma_{L}, \pi_{L}$ and $\eta_{M}$.

Our aim is to prove that the unit of each adjunction is the inverse of the counit of the other adjunction. This will establish that $\mathscr{R}$ and $\mathscr{I}$ are inverse equivalences.

(10.10) Lemma. $\pi_{L} \sigma_{L}=\mathrm{id}_{L}$.

Proof. We have $L_{*}(i)=j\left(L \uparrow_{N}^{G} \downarrow_{N}^{G}\right) \sigma_{L}$ because $L_{*}(i): L \rightarrow L \uparrow_{N}^{G} \downarrow_{N}^{G}$ factorizes through the inclusion $j\left(L \uparrow_{N}^{G} \downarrow_{N}^{G}\right): \mathscr{R} \mathscr{S}(L) \rightarrow L \uparrow_{N}^{G} \downarrow_{N}^{G}$. Therefore

$$
L^{*}(i) L_{*}(i)=L^{*}(i) j\left(L \uparrow_{N}^{G} \downarrow_{N}^{G}\right) \sigma_{L}=\pi_{L} \sigma_{L},
$$

and it suffices to show that $L^{*}(i) L_{*}(i)=\mathrm{id}_{L}$. This is a general property of Mackey functors. By the Mackey decomposition formula 5.3, we have

$$
Y \uparrow_{N}^{G} \downarrow_{N}^{G}=\bigcup_{g \in[N \backslash G / N]} g_{Y} \downarrow_{s_{N N \cap N}}^{g_{N}} \uparrow_{s_{N \cap N N}^{N}}^{N}=Y \cup Y^{\prime},
$$

where $Y^{\prime}$ is the direct sum running over all non-trivial double cosets. Moreover the map $i_{Y}: Y \rightarrow Y \uparrow_{N}^{G} \downarrow_{N}^{G}$ is just the inclusion into the first summand. By applying $L_{*}$, we see that

$$
L_{*}\left(i_{Y}\right): L(Y) \rightarrow L\left(Y \oplus Y^{\prime}\right)=L(Y) \oplus L\left(Y^{\prime}\right)
$$

is the inclusion into the first summand. Similarly,

$$
L^{*}\left(i_{Y}\right): L\left(Y \oplus Y^{\prime}\right)=L(Y) \oplus L\left(Y^{\prime}\right) \rightarrow L(Y)
$$

is the first projection. Therefore $L^{*}\left(i_{Y}\right) L_{*}\left(i_{Y}\right)=\mathrm{id}_{L(Y)}$ as required.

(10.11) Lemma. $\sigma_{L} \pi_{L}=\operatorname{id}_{\mathscr{R}}(L)$.

Proof. By Lemma 10.7, we know that $L \uparrow_{N}^{G}$ lies in $\operatorname{Mack}_{R}(G, J)$ and that

$$
\left.L \uparrow_{N}^{G}\right\rfloor_{N}^{G}=\bigoplus_{g \in\left[N \backslash T_{G}(J, N) / N\right]} L_{g}
$$

where $L_{g}$ is a Mackey functor in $\operatorname{Mack}_{R}\left(N, g_{J}\right)$. Moreover $L_{1}=\mathscr{R} \mathscr{I}(L)$. On the other hand, by the Mackey decomposition formula, we have

$$
L \uparrow_{N}^{G} \downarrow_{N}^{G}=\bigoplus_{g \in[N \backslash G / N]}{ }^{g} L \downarrow_{g_{N N \cap N} \uparrow^{g_{N}} \uparrow_{8 N \cap N}^{N}}^{N}
$$

Moreover the first term in this sum is isomorphic to $L$. Finally the inclusion of $L$ into $L \uparrow_{N}^{G} \downarrow_{N}^{G}$ followed by the projection onto $L_{1}=\mathscr{R} \mathscr{S}(L)$ is precisely the natural morphism $\sigma_{L}: L \rightarrow L_{1}$, which is a split injection by Lemma 10.10 . that

We claim that the two decompositions of $\left.L \uparrow_{N}^{G}\right\rfloor_{N}^{G}$ above coincide, in the sense

$$
{ }^{g} L \downarrow_{8 N \cap N}^{g_{N}} \uparrow_{{ }_{s N \cap N}^{N}}^{N}= \begin{cases}L_{g} & \text { if } g \in T_{G}(J, N), \\ 0 & \text { otherwise. }\end{cases}
$$


This implies in particular that the split injection $\sigma_{L}: L \rightarrow L_{1}$ is an isomorphism, which establishes the lemma.

To prove the claim, we note that by Lemma 10.7 (iv), $L \downarrow_{N \cap 8^{-1} N}^{N}=0$ whenever $g \notin T_{G}(J, N)$, while $L \downarrow_{N \cap g^{-1} N}^{N}$ lies in $\operatorname{Mack}_{R}\left(N \cap g^{-1} N, J\right)$ if $g \in$ $T_{G}(J, N)$. Conjugating by $g$ and using Lemma 10.7 (iii), we obtain that ${ }^{g} L \downarrow_{s N \cap N}^{g_{N}}=0$ if $g \notin T_{G}(J, N)$ and that ${ }^{g} L \downarrow_{g N \cap N}^{g_{N}}$ lies in $\operatorname{Mack}_{R}\left({ }^{g} N \cap N,{ }^{g} J\right)$ if $g \in T_{G}(J, N)$. Now ${ }^{g} N \cap N$ is the normalizer in $N$ of the $p$-perfect subgroup ${ }^{g} J$, so by Lemma 10.7 (i), we obtain that ${ }^{g} L \downarrow_{8 N \cap N}^{g_{N}} \uparrow_{{ }_{8 N \cap N}}^{N}$ lies in $\operatorname{Mack}_{R}\left(N, g_{J}\right)$. This proves that every summand in the second decompositon of $L \uparrow_{N}^{G} \downarrow_{N}^{G}$ above either is zero (when $g \notin T_{G}(J, N)$ ) or is exactly the summand belonging to $\operatorname{Mack}_{R}\left(N,{ }^{g} J\right)$ (when $g \in T_{G}(J, N)$ ). This completes the proof of the claim.

(10.12) Lemma. $\epsilon_{M} \eta_{M}=\mathrm{id}_{M}$.

Proof. We have to show that

$$
M \stackrel{M^{*}(p)}{\longrightarrow} M \uparrow_{N}^{G} \downarrow_{N}^{G} \stackrel{m\left(f_{J}^{N}\right) \uparrow_{N}^{G}}{\longrightarrow} M \uparrow_{N}^{G} \downarrow_{N}^{G} \stackrel{M_{*}(p)}{\longrightarrow} M
$$

is the identity. By Lemma 9.8, we know that this is equal to the action of the element $f_{J}^{N} \uparrow_{N}^{G}$ of $B(G)$. Now Lemma 10.5 (ii) asserts that $f_{J}^{N} \uparrow_{N}^{G}=f_{J}^{G}$, which by assumption acts as the identity on $M$.

There remains to show that $\eta_{M} \epsilon_{M}=\mathrm{id} \mathscr{\mathscr { R }}(M)$. But this is a consequence of the following general lemma, because we already know that $\epsilon_{M} \eta_{M}=\mathrm{id}_{M}$ and that $\pi_{L}$ is an isomorphism.

(10.13) Lemma. Let $\mathscr{A}$ and $\mathscr{B}$ be two categories. Let $\mathscr{R}: \mathscr{A} \rightarrow \mathscr{B}$ and $\mathscr{I}: \mathscr{B} \rightarrow \mathscr{A}$ be two functors such that $\mathscr{R}$ is the left adjoint of $\mathscr{I}$. Let $\eta: \mathrm{id}_{\mathscr{A}} \rightarrow \mathscr{I}$ and $\pi: \mathscr{R} \mathscr{S} \rightarrow \mathrm{id}_{\mathscr{B}}$ be the unit and counit of the adjunction. Suppose that:

(i) $\epsilon_{M} \eta_{M}=\operatorname{id}_{M}$ for each $M \in \mathscr{A}$, where $\epsilon_{M}: \mathscr{\mathscr { R }}(M) \rightarrow M$ is some morphism in $\mathscr{A}$.

(ii) $\pi_{L}$ is an isomorphism for each $L \in \mathscr{B}$.

Then $\eta_{M}$ is an isomorphism for each $M \in \mathscr{A}$ (and so the categories $\mathscr{A}$ and $\mathscr{B}$ are equivalent).

Proof. One of the consequences of the existence of an adjunction is that for each $M \in \mathscr{A}$, the composite

$$
\mathscr{R}(M) \stackrel{\mathscr{R}\left(\eta_{\mathcal{M}}\right)}{\longrightarrow} \mathscr{R} \mathscr{Y R}(M) \stackrel{\pi_{\mathscr{R}(M)}}{\longrightarrow} \mathscr{R}(M)
$$

is the identity. Since $\pi_{\mathscr{R}(M)}$ is an isomorphism by assumption (ii), we also have that $\mathscr{R}\left(\eta_{M}\right) \pi_{\mathscr{R}(M)}$ is the identity (of $\mathscr{R} \mathscr{\mathscr { R }}(M)$ ). Since $\epsilon_{M} \eta_{M}=\mathrm{id}_{M}$ by assumption (i), we have $\mathscr{R}\left(\epsilon_{M}\right) \mathscr{R}\left(\eta_{M}\right)=\mathrm{id}_{\mathscr{R}(M)}$ and therefore

$$
\mathscr{R}\left(\epsilon_{M}\right)=\mathscr{R}\left(\epsilon_{M}\right) \mathscr{R}\left(\eta_{M}\right) \pi_{\mathscr{R}(M)}=\pi_{\mathscr{R}(M)} .
$$

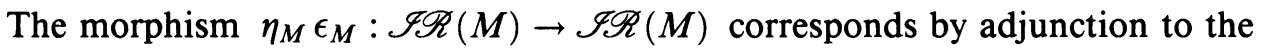
composite

$$
\pi_{\mathscr{R}(M)} \mathscr{R}\left(\eta_{M} \epsilon_{M}\right): \mathscr{R} \mathscr{\mathscr { R }}(M) \rightarrow \mathscr{R}(M),
$$


which by 10.14 is equal to

$$
\pi_{\mathscr{R}(M)} \mathscr{R}\left(\eta_{M}\right) \mathscr{R}\left(\epsilon_{M}\right)=\mathscr{R}\left(\epsilon_{M}\right)=\pi_{\mathscr{R}(M)}=\pi_{\mathscr{R}(M)} \mathscr{R}\left(\operatorname{id}_{\mathscr{R}(M)}\right) .
$$

But this is the correspondent of $\operatorname{id}_{\mathscr{R}(M)}$ by the adjunction, and it follows that

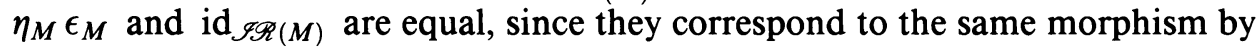
adjunction. This shows that $\epsilon_{M}$ is the inverse of $\eta_{M}$.

The proof of part (ii) of Theorem 10.1 is now complete.

\section{VERTICES, SOURCES AND GREEN CORRESPONDENTS OF PROJECTIVE AND SIMPLE MACKEY FUNCTORS}

In this section we will assume that the coefficient ring is a field $k$ (or more generally a complete discrete valuation ring). Recall from Sasaki [17] that in this situation every indecomposable Mackey functor $M$ has a vertex $\operatorname{vx}(M)$, namely a unique minimal subgroup (up to conjugacy) relative to which it is projective. Sasaki also proved that Green correspondence works in this situation, so that if $H=\operatorname{vx}(M)$ and $K$ is a subgroup containing $N_{G}(H)$ then $M \downarrow_{K}^{G}$ has a unique summand $f(M)$ with vertex $H$, and if $L$ is a Mackey functor for $K$ with vertex $H$ then $L \uparrow_{K}^{G}$ has a unique summand $g(L)$ with vertex $H$, the correspondences $f$ and $g$ being mutually inverse.

We will identify completely the vertices, sources and Green correspondents mentioned in the title. Coming from the background of group representation theory one would be inclined to think that the projective Mackey functors are the same as the Mackey functors which are projective relative to the identity subgroup, but this is not so. If $M$ is 1-projective (over a field), then it is indeed the case that $M$ is projective, but the converse does not hold. In fact for every subgroup $H$ of $G$ there is a projective Mackey functor whose vertex is $H$, as our first result shows.

Throughout this section, we shall often use superscripts to indicate for which group a Mackey functor is considered. Thus for instance $S_{H, V}^{K}$ is the simple Mackey functor for $K$ corresponding to the pair $(H, V)$.

(11.1) Proposition. Let $H \leq G$ and let $V$ be a simple $k \bar{N}_{G}(H)$-module.

(i) The indecomposable projective $P_{H, V}$ has vertex $H$.

(ii) Let $J=O^{p}(H)$ and let $f_{J}$ be the idempotent of the Burnside algebra $B(H)$ corresponding to $J$ (as in Theorem 9.3). Then $P_{H, k}^{H}=f_{J} \cdot B^{H}$ is a source of $P_{H, V}$. In particular, if $H$ is a p-group, then $B^{H}$ is a source of $P_{H, V}$.

Proof. (i) Since $P_{H, V}$ is a summand of $B^{H} \uparrow_{H}^{G}$ by 8.7(ii), the subgroup $H$ contains a vertex of $P_{H, V}$. Suppose that $P_{H, V}$ is a summand of $M \uparrow_{K}^{G}$ for some Mackey functor $M$, where $K<H$. Then there exists a non-zero morphism $M \uparrow_{K}^{G} \rightarrow S_{H, V}$ and hence a non-zero morphism $M \rightarrow S_{H, V} \downarrow_{K}^{G}$ by adjointness of induction and restriction. But from the description of the simple Mackey functors, $S_{H, V} \downarrow_{K}^{G}$ is the zero Mackey functor, a contradiction.

(ii) Since $P_{H, V}$ is a summand of $B^{H} \uparrow_{H}^{G}$, the source of $P_{H, V}$ has the form $f \cdot B^{H}$ for some idempotent $f$ of $B(H)$, by 9.3. By the argument of (i), there is a non-zero morphism $f \cdot B^{H} \rightarrow S_{H, V} \downarrow_{H}^{G}$. But $S_{H, V} \downarrow_{H}^{G}$ vanishes below $H$, hence is a direct sum of copies of $S_{H, k}^{H}$. Therefore the projective Mackey 
functor $f \cdot B^{H}$ must be the projective cover of $S_{H, k}^{H}$ and this means that $f=f_{J}$ by 9.11 . Finally, in case $H$ is a $p$-group, then $f_{1}$ is already primitive in $B(H)$, by 9.3 (or 8.10 ), and $f_{1} \cdot B^{H}=B^{H}$.

Now we consider Green correspondents of projective Mackey functors.

(11.2) Proposition. Let $K$ be a subgroup of $G$ which contains $N_{G}(H)$. The Green correspondent of $P_{H, V}^{G}$ is $P_{H, V}^{K}$.

Proof. We may write $P_{H, V}^{K} \uparrow_{K}^{G} \cong g\left(P_{H, V}^{K}\right) \oplus M$ where $M$ is a Mackey functor all of whose summands have smaller vertex than $H$. Since induction preserves projectives, by the last proposition these summands have the form $P_{L, W}$ with $L<H$. Applying induction to the epimorphism $P_{H, V}^{K} \rightarrow S_{H, V}^{K}$ gives an epimorphism $g\left(P_{H, V}^{K}\right) \oplus M \cong P_{H, V}^{K} \uparrow_{K}^{G} \rightarrow S_{H, V}^{G}$, using the fact that $S_{H, V}^{G}$ is an induced functor. The only way we can have such an epimorphism to a simple Mackey functor is if $P_{H, V}^{G}$ is a summand, and since this has vertex $H$ we must have $g\left(P_{H, V}^{K}\right)=P_{H, V}^{G}$.

We turn now to the vertices and sources of simple Mackey functors. We will need to quote Proposition 3.4.2 of [21], whose statement we now include.

(11.3) Lemma. Suppose that $M$ is a Mackey functor which is projective relative to a set of subgroups $\mathscr{Z}$, and let $\mathscr{Y}$ be a set of subgroups of $G$ which is closed under taking subgroups and conjugation. Consider the Mackey functor $N$ whose value at a subgroup $H$ is defined to be $N(H)=\sum_{J \leq H, J \in \mathscr{Y}} I_{J}^{H} M(J)$. Then $N$ is also projective relative to $\mathscr{Z}$.

We also need to extend the notation $S_{H, V}$ to non-simple modules $V$. If $V$ is an arbitrary $k \bar{N}(H)$-module, define $S_{1, V}^{\bar{N}(H)}(K)=\operatorname{tr}_{1}^{K}(V) \subseteq V^{K}=F P_{V}(K)$. Thus $S_{1, V}$ is a subfunctor of $F P_{V}$. Then define

$$
S_{H, V}=S_{H, V}^{G}=\left(\operatorname{Inf}_{\bar{N}(H)}^{N(H)} S_{1, V}^{\bar{N}(H)}\right) \uparrow_{N(H)}^{G} .
$$

It is not difficult to see that $S_{H, V}$ is generated by $S_{H, V}(H)=V$. This follows exactly as in the proof of part (i) of Lemma 8.1 in [20]. A consequence of this fact is that $S_{H, V}$ is indecomposable if and only if $V$ is an indecomposable $k \bar{N}(H)$-module, using also Proposition 3.2 of [20].

\section{(11.4) Proposition.}

(i) For any indecomposable $k G$-module $W$, the vertices of $F P_{W}, F Q_{W}$ and $W$ are the same.

(ii) Let $S_{H, V}^{G}$ be a simple Mackey functor, and let $K$ be a subgroup of $G$ with $H \leq K \leq N_{G}(H)$ such that $K / H$ is a vertex of $V$. Then $K$ is a vertex of $S_{H, V}^{G}$.

(iii) Let moreover $U$ be a source of $V$. Then the indecomposable Mackey functor $S_{H, U}^{K}$ is a source of $S_{H, V}^{G}$.

Proof. (i) We rely on the fact that induction of Mackey functors commutes with all of $F P, F Q$ and evaluation at 1 . Let $K$ be a vertex of $W$. Then since $W$ is a summand of $W \uparrow_{K}^{G}$ we have that $F P_{W}$ is a summand of $F P_{W \dagger_{K}^{G}} \cong$ $F P_{W} \uparrow_{K}^{G}$. Hence $K$ contains a vertex $L$ of $F P_{W}$. On the other hand, the split 
epimorphism $F P_{W} \uparrow_{L}^{G} \rightarrow F P_{W}$ on evaluation at the identity subgroup is a split epimorphism $W \uparrow_{L}^{G} \rightarrow W$, and hence $L$ must be $K$. The proof for $F Q_{W}$ is similar.

(ii) Since $S_{H, V}^{G}=\left(\operatorname{Inf}_{\bar{N}(H)}^{N(H)} S_{1, V}^{\bar{N}(H)}\right) \uparrow_{N(H)}^{G}$ is an induced functor, it follows that a vertex of $\operatorname{Inf}_{\bar{N}(H)}^{N(H)} S_{1, V}^{\bar{N}(H)}$ as a Mackey functor for $N(H)$ is also a vertex of $S_{H, V}^{G}$. Evidently this will be the preimage in $N(H)$ of a vertex of $S_{1, V}^{\bar{N}(H)}$. By this means we reduce to the case that $H=1$, so we consider a simple Mackey functor $S_{1, V}$.

A vertex $L$ of $S_{1, V}$ must always contain a vertex $K$ of $V$ since the split epimorphism $S_{1, V} \downarrow_{L}^{G_{\uparrow} G} \rightarrow S_{1, V}$ on evaluation at 1 gives a split epimorphism $V \downarrow_{L}^{G} \uparrow_{L}^{G} \rightarrow V$. On the other hand $F P_{V}$ is projective relative to $K$ by part (i) and $S_{1, V}$ is constructed as in 11.3 on taking $M=F P_{V}, \mathscr{Y}=\{1\}$ so it follows by 11.3 that $S_{1, V}$ is also projective relative to $K$. Thus $L=K$.

(iii) By the same reduction argument as in (ii), we are left with the case $H=1$. Since $V$ is a summand of $U \uparrow_{K}^{G}, F P_{V}^{G}$ is a summand of $F P_{U \uparrow_{K}^{G}}^{G} \cong$ $F P_{U}^{K} \uparrow_{K}^{G}$. Now by the definition of $S_{1, V}^{G}$, it is clear that $S_{1, V}^{G}$ is a summand of $S_{1, U\}_{K}^{G}}^{G}=S_{1, U}^{K} \uparrow_{K}^{G}$. The detailed proof of the latter equality is left to the reader. Since we already know that $K$ is a vertex of $S_{1, V}^{G}$, the argument suffices to guarantee that $S_{1, U}^{K}$ is a source of $S_{1, V}^{G}$.

Finally we show that the Green correspondents of simple Mackey functors are determined by the Green correspondents of the corresponding modules. We first need a lemma.

(11.5) Lemma. Let $K$ be a normal subgroup of $G$ and let $K \leq J \leq G$. Let $M$ be an indecomposable Mackey functor for $J$ with vertex $K$. Then every indecomposable summand of $M \uparrow_{J}^{G}$ has vertex $K$.

Proof. Let $N$ be a source of $M$. Since $M$ is a summand of $N \uparrow_{K}^{J}$, the functor $M \downarrow_{K}^{J}$ is a summand of

$$
N \uparrow_{K}^{J} \downarrow_{K}^{J}=\bigoplus_{h \in[J / K]}{ }^{h} N
$$

Thus $M \downarrow_{K}^{J}$ is a direct sum of conjugates of $N$.

Now let $L$ be an indecomposable summand of $M \uparrow_{J}^{G}$. Then $L \downarrow_{K}^{G}$ is a summand of

$$
M \uparrow_{J}^{G} \downarrow_{K}^{G}=\bigoplus_{g \in[K \backslash G / J]} g_{M} \downarrow_{K}^{g J}=\bigoplus_{g \in[G / J]}{ }^{g}\left(M \downarrow_{K}^{J}\right)
$$

and so every summand of $L \downarrow_{K}^{G}$ is a conjugate of $N$. After conjugation, it follows that $N$ is a summand of $L \downarrow_{K}^{G}$. Since we also have that $L$ is a summand of $N \uparrow_{K}^{G}$ and since $N$ is its own source, $N$ must be a source of $L$. In particular $K$ is a vertex of $L$.

(11.6) Proposition. Let $S_{H, V}^{G}$ be a simple Mackey functor for $G$ and let $K / H$ be a vertex of $V$. Let $W$ be the Green correspondent of $V$, a module for $N_{N(H) / H}(K / H)=N(H, K) / H$, where $N(H, K)=N(H) \cap N(K)$. Then the 
Mackey functor for $N(K)$ which is the Green correspondent of $S_{H, V}^{G}$ is equal to $S_{H, W}^{N(H, K)} \uparrow_{N(H, K)}^{N(K)}$.

Proof. The functor $V \mapsto S_{1, V}$ from modules to Mackey functors is additive and the proof of 11.4 shows that it preserves vertices. It follows easily that $S_{1, W}^{N(H, K) / H}$ is the Green correspondent of $S_{1, V}^{N(H) / H}$. Applying inflation from $N(H) / H$ to $N(H)$, one obtains that $S_{H, W}^{N(H, K)}$ is the Green correspondent of $S_{H, V}^{N(H)}$. Therefore

$$
S_{H, W}^{N(H, K)} \uparrow_{N(H, K)}^{N(H)} \cong S_{H, V}^{N(H)} \oplus T
$$

where each summand of $T$ has vertex smaller than $K$.

Let $M=S_{H, W}^{N(H, K)} \uparrow_{N(H, K)}^{N(K)}$. Then

$$
M \uparrow_{N(K)}^{G}=S_{H, W}^{N(H, K)} \uparrow_{N(H, K)}^{G} \cong S_{H, V}^{G} \oplus T \uparrow_{N(H)}^{G} .
$$

By 11.5 , every indecomposable summand of $M$ has vertex $K$. By Green correspondence, each such summand corresponds to an indecomposable summand of $M \uparrow_{N(K)}^{G}$ with vertex $K$. But we have just seen that $S_{H, V}^{G}$ is the only summand of $M \uparrow_{N(K)}^{G}$ with vertex $K$. It follows that $M$ is indecomposable and (*) shows that $M$ is the Green correspondent of $S_{H, V}^{G}$.

\section{Projective Mackey functors INDEXed by $p$-SUbGRoups}

In this section our coefficient ring will be a field $k$ of characteristic $p>0$. We analyse the relationship between the projective Mackey functors $P_{H, V}$ where $H$ is a $p$-group, and their evaluations at the trivial subgroup 1 . This provides a bijection between these projectives and indecomposable trivial source modules, i.e., the indecomposable summands of permutation modules. Also there is a strong connection with Hecke algebras, by which we mean the $k$ algebras $\operatorname{End}_{k G}\left(k \uparrow_{H}^{G}\right)$.

For completeness, we mention that the case of arbitrary projective Mackey functors reduces to the situation analysed in this section, thanks to the equivalence of categories 10.1. Indeed if $P_{H, V}^{G}$ is the projective cover of $S_{H, V}^{G}$, then by 9.6, $S_{H, V}^{G}$ lies in $\operatorname{Mack}_{k}(G, J)$ where $J=O^{p}(H)$ and therefore so does $P_{H, V}^{G}$. By the equivalence $10.1, S_{H, V}^{G}$ corresponds to $S_{H / J, V}^{\bar{N}(J)}$ (because we have $S_{H, V}^{G}=S_{H, V}^{N(H)} \uparrow_{N(H)}^{G}$ ) and since $N(H) \leq N(J)$, we obtain

$$
S_{H, V}^{G}=S_{H, V}^{N(J)} \uparrow_{N(J)}^{G}=\left(\operatorname{Inf}_{\bar{N}(J)}^{N(J)} S_{H / J, V}^{\bar{N}(J)}\right) \uparrow_{N(J)}^{G} .
$$

Therefore $P_{H, V}^{G}$ corresponds to $P_{H / J, V}^{\bar{N}(J)}$ under the equivalence and since $H / J$ is a $p$-group, we are in the situation of this section.

The following lemma is well known, but we give the proof for the sake of completeness.

(12.1) Lemma. Let $H$ be a p-group. Then $\overline{F P_{k \uparrow} G}(H) \cong k \bar{N}_{G}(H)$ as $k \bar{N}_{G}(H)$ modules.

Proof. We have a decomposition $k \uparrow_{H}^{G} \downarrow_{H}^{G}=\bigoplus_{x \in[H \backslash G / H]} k \uparrow_{H^{x} \cap H}^{H}$ and each summand has a 1-dimensional fixed point set which is spanned by $\operatorname{tr}_{H^{x} \cap H}^{H} 1$. In 
$\overline{F P_{k\}_{H}^{G}}}(H)$ there remain only the terms which are not traces from proper subgroups. These arise when $H^{x} \cap H=H$, i.e., $x \in N_{G}(H)$, so $\overline{F P_{k\rceil_{H}^{G}}}(H)=$ $\bigoplus_{x \in\left[N_{G}(H) / H\right]} k$ and the action of $N_{G}(H) / H$ on these summands is to permute them regularly.

(12.2) Corollary. Let $H$ be a p-subgroup of $G$ and $V$ a simple $k \bar{N}_{G}(H)$ module.

(i) The simple Mackey functor $S_{H, V}$ always occurs as a composition factor of $F P_{k \uparrow_{H}^{G}}$.

(ii) $P_{H, V}(1) \neq 0$. There exists a simple $k G$-module $W$ such that $S_{1, W}$ is a composition factor of $P_{H, V}$. Equivalently, $S_{H, V}$ is a composition factor of $P_{1, W}$.

Proof. (i) Every simple $k \bar{N}_{G}(H)$-module $V$ is a composition factor of $k \bar{N}_{G}(H)$ and so by applying Proposition 6.2 we obtain that $S_{H, V}$ is a composition factor of $F P_{k \uparrow_{H}^{G}}$.

(ii) By (i) there exists a non-zero morphism $P_{H, V} \rightarrow F P_{k \uparrow_{H}^{G}}$. By the adjoint property of the fixed point functor this means that there exists a non-zero morphism $P_{H, V}(1) \rightarrow k \uparrow_{H}^{G}$, and so we must have $P_{H, V}(1) \neq 0$. Now if $W$ is any composition factor of $P_{H, V}(1)$, it follows by 6.3 that $S_{1, W}$ is a composition factor of $P_{H, V}$. The final assertion follows by symmetry of the Cartan matrix.

(12.3) Lemma. For any subgroup $H$ there is an isomorphism

$$
B^{H} \uparrow_{H}^{G}(1) \cong k \uparrow_{H}^{G}
$$

as $k G$-modules.

Proof. This is a consequence of the facts that $B^{H}(1)=B(1) \cong k$ and that induction commutes with evaluation at 1 by 5.2 .

(12.4) Lemma. For any projective Mackey functor $P$, the morphisms $F Q_{P(1)} \rightarrow$ $P$ and $P \rightarrow F P_{P(1)}$ which extend the identity map at the level of the identity subgroup are, respectively, a monomorphism and an epimorphism.

Proof. We prove that the first of these morphisms is a monomorphism. It suffices to assume $P$ is indecomposable. Since $P$ is a direct summand of one of the functors $B^{H} \uparrow_{H}^{G}$, it suffices to prove the result when $P=B^{H} \uparrow_{H}^{G}$, and

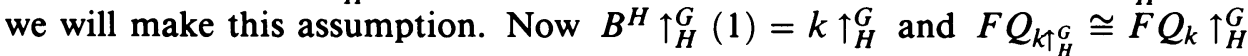
by 5.2, so the first of these morphisms is $F Q_{k} \uparrow_{H}^{G} \rightarrow B^{H} \uparrow_{H}^{G}$. We claim that this morphism is $\left(F Q_{k} \rightarrow B^{H}\right) \uparrow_{H}^{G}$. This is because both morphisms are uniquely determined by their effect at the identity subgroup, and at that level they are both the identity morphism. Because $\uparrow_{H}^{G}$ is an exact functor it suffices to show that the morphism $F Q_{k} \rightarrow B^{H}$ of Mackey functors for $H$ is a monomorphism. But at the level of a subgroup $K \leq H$ this morphism is

$$
\begin{aligned}
F Q_{k}(K) & \rightarrow B^{H}(K), \\
1 & \mapsto K / 1
\end{aligned}
$$

since in the functor $F Q_{k}$ we have $I_{1}^{K}(1)=1$. This is evidently injective. 
The argument that the second morphism is an epimorphism is similar. We reduce to showing that $B^{H} \rightarrow F P_{k}$ is an epimorphism of Mackey functors for $H$. At the level of a subgroup $K \leq H$ this morphism is

$$
\begin{aligned}
B^{H}(K) & \rightarrow F P_{k}(K), \\
K / J & \mapsto|K / J|
\end{aligned}
$$

which is seen to be surjective because $K / K$ maps to 1 .

(12.5) Corollary. For every indecomposable projective Mackey functor $P$, the $k G$-module $P(1)$ is indecomposable.

Proof. Suppose $P(1)=A \oplus B$ with $A$ and $B$ non-zero. Then $F P_{P(1)}=$ $F P_{A} \oplus F P_{B}$. But $P \rightarrow F P_{P(1)}$ is epi and any image of $P$ is indecomposable since it has a simple top. Hence $P(1)$ cannot decompose.

Our aim at the moment is to prove Theorem 12.7 , but as a preliminary we first prove a special case.

(12.6) Lemma. Let $P_{H, V}$ be an indecomposable projective Mackey functor where $H$ is a normal p-subgroup of $G$. Then $P_{H, V}(1)$ is the projective cover as a $k[G / H]$-module of $V$, inflated to $G$. In particular, $P_{H, V}$ and $P_{H, V}(1)$ both have vertex $H$.

Proof. $P_{H, V}(1)$ is an indecomposable summand of

$$
B^{H} \uparrow_{H}^{G}(1)=k \uparrow_{H}^{G}=k[G / H],
$$

and the summands of this module are exactly the projective covers over $k[G / H]$ of the simple $k[G / H]$-modules, so $P_{H, V}(1)$ is one of these. We show that there is an epimorphism $P_{H, V}(1) \rightarrow V$ as $N_{G}(H) / H$-modules, and that will show that $P_{H, V}(1)$ is the projective cover of $V$. We do know from 12.4 that the unique simple image of $P_{H, V}$ as a Mackey functor is also an image of $F P_{P_{H}, V}(1)$, so we have an epimorphism $F P_{P_{H, V}(1)} \rightarrow S_{H, V}$. Evaluating this at $H$ we obtain an epimorphism

$$
P_{H, V}(1)^{H}=P_{H, V}(1) \rightarrow V
$$

as required.

(12.7) Theorem. Let $P_{H, V}$ be an indecomposable projective Mackey functor.

(i) If $H$ is not a p-group then $P_{H, V}(1)=0$.

(ii) If $H$ is a p-group then $P_{H, V}(1)$ is a non-zero indecomposable summand of $k \uparrow_{H}^{G}$. In fact $P_{H, V}(1)$ is the trivial source module with vertex $H$ which is the Green correspondent $g\left(P_{V}\right)$, where $P_{V}$ is the projective cover of $V$ as a $k \bar{N}_{G}(H)$-module, which we regard as a $k N_{G}(H)$-module by inflation.

Proof. (i) The projective $P_{H, V}$ lies in $\operatorname{Mack}_{k}\left(G, O^{p}(H)\right)$ by 11.1(i) and 9.7, and so if $H$ is not a $p$-group then $P_{H, V}(1)=0$ by 9.5 .

(ii) Since the Green correspondent of $P_{H, V}^{G}$ is $P_{H, V}^{N_{G}(H)}$ by 11.2, we have $P_{H, V}^{N_{G}(H)} \uparrow_{N_{G}(H)}^{G} \cong P_{H, V}^{G} \oplus M$ where $M$ is a Mackey functor all of whose summands have vertex smaller than $H$. Thus $P_{H, V}^{N_{G}(H)} \uparrow_{N_{G}(H)}^{G}(1)=P_{H, V}^{G}(1) \oplus M(1)$ and 
since induction commutes with evaluation at $1, M(1)$ is a module all of whose summands have vertex smaller than $H$. Furthermore we may write the lefthand side of the last equation as $\left(P_{H, V}^{N_{G}(H)}(1)\right) \uparrow_{N_{G}(H)}^{G}$. Because $H$ is a normal p-subgroup of $N_{G}(H)$, Lemma 12.6 applies and we have $P_{H, V}^{N_{G}(H)}(1)=P_{V}$, the projective cover of $V$. Now $P_{V} \uparrow_{N_{G}(H)}^{G}=g\left(P_{V}\right) \oplus A$, where $A$ is a module all of whose summands have vertex smaller than $H$. Combining the above, we have an equation

$$
P_{H, V}^{G}(1) \oplus M(1)=g\left(P_{V}\right) \oplus A
$$

and equating the only two modules which have vertex $H$ we obtain $P_{H, V}^{G}(1) \cong$ $g\left(P_{V}\right)$ as required.

(12.8) Corollary. Let $H$ be a p-subgroup of $G$.

(i) The vertices of $P_{H, V}$ and $P_{H, V}$ (1) are the same.

(ii) $P_{H, V} \cong P_{K, W}$ as Mackey functors if and only if $P_{H, V}(1) \cong P_{K, W}(1)$ as $k G$-modules.

Proof. (i) The vertex of $P_{H, V}(1)$ is $H$. By 11.1 this is also the vertex of $P_{H, V}$.

(ii) If $P_{H, V}(1) \cong P_{K, W}(1)$ as $k G$-modules then $K$ and $H$ are conjugate since they are vertices of the module concerned. Now $V$ and $W$ are determined as the unique simple image of a Green correspondent of $P_{H, V}(1)$, and hence they are isomorphic.

(12.9) Corollary. Let $H$ be a p-subgroup of $G$. The indecomposable summands of $B^{H} \uparrow_{H}^{G}$ biject with the indecomposable summands of $k \uparrow_{H}^{G}$. In fact if $B^{H} \uparrow_{H}^{G}=\bigoplus P_{i}$ is a direct sum decomposition into indecomposable projective Mackey functors then $k \uparrow_{H}^{G}=\bigoplus P_{i}(1)$ is a direct sum decomposition into indecomposable $k G$-modules.

Proof. If $P_{K, W}$ is an indecomposable summand of $B^{H} \uparrow_{H}^{G}$ then $K$ is conjugate to a subgroup of $H$ by $8.7(\mathrm{i})$, so is a $p$-group. Thus $P_{K, W}(1)$ is a non-zero indecomposable $k G$-module by 12.2 and 12.5 , and the result follows.

As an example of this connection between trivial source modules and Mackey functors we state a result due to Alperin, having to do with weights. In this context a weight is a pair $(H, V)$ where $H$ is a $p$-subgroup of $G$ and $V$ is a projective $k \bar{N}_{G}(H)$-module.

(12.10) Proposition. Let $k$ be a field of characteristic $p$ and let $Q$ be a Sylow p-subgroup of $G$. Suppose that $(H, V)$ is a weight for $G$. Then

(i) $P_{H, V}$ is a summand of $B^{Q} \uparrow_{Q}^{G}$ with non-zero multiplicity.

(ii) ([2, Lemma 1]) Regarding $V$ as a $k N_{G}(H)$-module by inflation, the Green correspondent $g(V)$ is a summand of $k \uparrow_{Q}^{G}$ with non-zero multiplicity.

Proof. (i) By virtue of 8.6 we need to show that $S_{H, V}(Q) \neq 0$, which we do using 8.8. Since $H$ is a $p$-subgroup, there exists $g \in G$ with $H^{g} \subseteq Q$ and it suffices to show that $\operatorname{tr}_{1}^{N_{g_{Q}}(H) / H}(V) \neq 0$. But $N_{g_{Q}}(H) / H$ is a $p$-group so, being projective, $V$ is a direct sum of copies of $k\left[N_{g_{Q}}(H) / H\right]$, from which it follows that the trace from the identity is non-zero. 
(ii) follows from (i) by evaluation at 1 using 12.7(ii).

We now make the connection with Hecke algebras.

(12.11) Theorem. Let $H$ be a p-subgroup of $G$. The ring homomorphism

$$
s: \operatorname{End}_{\operatorname{Mack}_{k}(G)}\left(B^{H} \uparrow_{H}^{G}\right) \rightarrow \operatorname{End}_{k G}\left(k \uparrow_{H}^{G}\right)
$$

given by evaluation at 1 is surjective. Its kernel lies in the radical of End $_{\text {aack }_{k}(G)}\left(B^{H} \uparrow_{H}^{G}\right)$. It follows that every idempotent of $\operatorname{End}_{k G}\left(k \uparrow_{H}^{G}\right)$ lifts through $S$ to an idempotent of $\operatorname{End}_{\operatorname{Mack}_{k}(G)}\left(B^{H} \uparrow_{H}^{G}\right)$.

Proof. Evidently every Mackey functor endomorphism of $B^{H} \uparrow_{H}^{G}$ at the level of the subgroup 1 is a $k G$-module endomorphism of $B^{H} \uparrow_{H}^{G}(1) \cong k \uparrow_{H}^{G}$, and this gives the definition of $s$.

We show that $s$ is surjective. As abelian groups there is a decomposition

$$
\begin{aligned}
\operatorname{Hom}\left(B^{H} \uparrow_{H}^{G}, B^{H} \uparrow_{H}^{G}\right) & \cong \operatorname{Hom}\left(B^{H}, B^{H} \uparrow_{H}^{G} \downarrow_{H}^{G}\right) \\
& \cong \bigoplus_{x \in[H \backslash G / H]} \operatorname{Hom}\left(B^{H}, c_{x}\left(B^{H} \downarrow_{H^{x} \cap H}^{H}\right) \uparrow_{H \cap x_{H}}^{H}\right) \\
& \cong \bigoplus_{x \in[H \backslash G / H]} \operatorname{Hom}\left(B^{H} \downarrow_{H \cap x_{H}}^{H}, B^{H} \downarrow_{H \cap x_{H}}^{H}\right) \\
& \cong \bigoplus_{x \in[H \backslash G / H]} \operatorname{Hom}\left(B^{H \cap^{x} H}, B^{H \cap^{x} H}\right) .
\end{aligned}
$$

There is a similar decomposition

$$
\operatorname{Hom}\left(k_{H} \uparrow_{H}^{G}, k_{H} \uparrow_{H}^{G}\right) \cong \bigoplus_{x \in[H \backslash G / H]} \operatorname{Hom}(k, k)
$$

of $\operatorname{End}_{k G}\left(k \uparrow_{H}^{G}\right)$ which gives the basis for this endomorphism ring attributed to Schur. The ring homomorphism $s$ respects the terms in these decompositions, and on each summand it is the surjection $\operatorname{End}\left(B^{H \cap^{x} H}\right) \rightarrow \operatorname{End}(k)$ which sends the identity mapping to the identity. Evidently this is surjective.

We now show that the kernel of $s$ is contained in the radical. This follows from the fact that the kernel contains no non-zero idempotent: for if the kernel did contain a non-zero idempotent, the idempotent would correspond to a summand of $B^{H} \uparrow_{H}^{G}$ with zero evaluation at 1. By 12.9 this cannot happen.

We conclude this section with a remark that the connection between trivial source modules and projective Mackey functors allows us to give a proof of the well-known fact that trivial source modules are liftable from characteristic $p$ to characteristic 0 . Namely, if $\mathscr{O}$ is a complete discrete valuation ring with residue field $k$ of characteristic $p$, then any indecomposable trivial source $k G$ module $T$ is a summand of $k \uparrow_{H}^{G}$ where $H=\operatorname{vx}(T)$. Thus $T=P_{H, V}(1)$ for $V$ as specified by 12.7 , and now $\hat{P}_{H, V}(1)$ is an $\mathscr{O} G$-module which lifts $T$, using the fact that projectives lift.

\section{Projective Mackey functors indexed by the tRivial SUbGroup}

Throughout this section we will work with Mackey functors defined over a field $k$ of characteristic $p$. Our main result asserts that for each simple $k G$ module $V$ we have $P_{1, V} \cong F P_{P_{V}} \cong F Q_{P_{V}}$ where $P_{V}$ denotes the projective 
cover of $V$ as a $k G$-module. This immediately gives us a direct way to compute the structure of these Mackey functors, and we may also deduce some general facts, for example that $P_{1, V}$ is an injective Mackey functor, as well as a projective one. This is not the case for arbitrary projectives $P_{H, V}$ as we shall see in Section 19. The result may be extended in two directions, the first being to the case when $R=\mathscr{O}$ is a complete discrete valuation ring with residue field $k$. We may conclude that $\hat{P}_{1, V} \cong F P_{\hat{P}_{V}} \cong F Q_{\hat{P}_{V}}$ using hats to denote the projective covers over the discrete valuation ring, since $F P_{\hat{P}_{V}}$ and $F Q_{\hat{P}_{V}}$ are liftings to $\mathscr{O}$ of $F P_{P_{V}}$ and $F Q_{P_{V}}$. The other direction in which the result can be immediately extended is to a description of the projectives $P_{J, V}$ where $J$ is a $p$-perfect subgroup of $G$. We use the equivalence of categories given in 10.1 to deduce that $P_{J, V}=\left(\operatorname{Inf}_{\bar{N}(J)}^{N(J)} F P_{P_{V}}\right) \uparrow_{N(J)}^{G}$, where now $P_{V}$ denotes the projective cover of $V$ as a $k \bar{N}(J)$-module.

(13.1) Proposition. Let $k$ be a field, and let $V$ be a $k G$-module. Conditions (i)-(vi) are equivalent.

(i) $V$ is a projective module (or equivalently an injective module).

(ii) $F P_{V} \cong F Q_{V}$.

(iii) $F P_{V}$ is an injective Mackey functor.

(iv) $F Q_{V}$ is a projective Mackey functor.

(v) $F P_{V}$ is a projective Mackey functor.

(vi) $F Q_{V}$ is an injective Mackey functor.

If we suppose further that $V$ is a simple module, then (i)-(vi) are equivalent to:

(vii) $S_{1, v}=F P_{V}$.

(viii) $S_{1, V}$ is a projective Mackey functor.

(ix) $S_{1, V}$ is an injective Mackey functor.

Proof. (i) $\Rightarrow$ (ii) There is an isomorphism $\nu: F Q_{V} \rightarrow F P_{V}$ given at each subgroup $H$ by $\nu_{H}=\sum_{h \in H} h: V_{H} \rightarrow V^{H}$. It is well known that $\nu_{H}$ is an isomorphism for each subgroup $H$. Also one checks that $\nu$ is a natural transformation of Mackey functors.

(ii) $\Rightarrow$ (i) There is nothing to prove unless $k$ has characteristic $p>0$, so we will assume this. The induction maps for $F Q_{V}$ are all surjective, which means that if we have an isomorphism $F P_{V} \cong F Q_{V}$ then the induction map $I_{1}^{H}=\sum_{h \in H} h$ for $F P_{V}$ is always surjective. In particular this holds when $H$ is a Sylow $p$-subgroup of $G$ so that, as is well-known, $V$ is projective on restriction to a Sylow $p$-subgroup, and hence $V$ is projective as a $k G$-module.

(i) $\Rightarrow$ (iii) Suppose that $\theta: F P_{V} \rightarrow M$ is a monomorphism of Mackey functors. We construct a splitting as follows. Evaluating at the identity subgroup gives a monomorphism $V \rightarrow M(1)$, which is split by some map $\eta(1): M(1) \rightarrow$ $V$ since $V$ is injective. By adjointness this extends to a map $\eta: M \rightarrow F P_{V}$ such that the composite $\eta \theta$ is the identity on $V=F P_{V}(1)$. But any endomorphism of $F P_{V}$ is determined by its effect on $V$, so $\eta \theta=1$.

(iii) $\Rightarrow$ (i) Suppose we are given a module monomorphism $V \rightarrow W$. This extends to a Mackey functor morphism $F P_{V} \rightarrow F P_{W}$ which is a monomorphism since for every subgroup $H, V^{H} \rightarrow W^{H}$ is injective. Since $F P_{V}$ is injective the morphism of Mackey functors splits. At the level of the identity subgroup this gives a splitting of the monomorphism $V \rightarrow W$. Hence $V$ is injective, and also projective. 
(i) $\Leftrightarrow$ (iv) is similar to (i) $\Leftrightarrow$ (iii).

(i) $\Rightarrow$ (v) Suppose we have an epimorphism of Mackey functors $\theta: M \rightarrow$ $F P_{V}$. We construct a splitting as follows. Evaluating at the identity subgroup gives an epimorphism $M(1) \rightarrow V$, which is split by some map $\eta(1): V \rightarrow M(1)$ since $V$ is projective. By adjointness this extends to a map $\eta: F Q_{V} \rightarrow M$, and now by part (ii) we have an isomorphism $\nu: F Q_{V} \rightarrow F P_{V}$ which is also the identity at 1 , so we have $\eta \nu^{-1}: F P_{V} \rightarrow M$ such that the composite $\theta \eta \nu^{-1}$ is the identity on $V=F P_{V}(1)$. But any endomorphism of $F P_{V}$ is determined by its effect on $V$, so $\theta \eta \nu^{-1}=1$.

(v) $\Rightarrow$ (i) Suppose that $F P_{V}$ is projective. The natural map $F Q_{V} \rightarrow F P_{V}$ is injective by 12.4 , so that $F Q_{V}$ is isomorphic to a subfunctor of $F P_{V}$. It follows that the restriction morphisms $R_{1}^{H}$ for $F Q_{V}$ are injective for every subgroup $H$ of $G$, since the same is true of $F P_{V}$. But $R_{1}^{H}$ is the morphism on fixed quotients induced by $\sum_{h \in H} h$, and it is well-known that these morphisms are all injective if and only if $V$ is projective.

(i) $\Leftrightarrow$ (vi) follows from (i) $\Leftrightarrow$ (v) and duality.

(i) $\Leftrightarrow$ (vii) Both conditions are equivalent to the condition that the transfer map $I_{1}^{H}: V \rightarrow V^{H}$ is surjective for all subgroups $H$.

(i) $\Rightarrow$ (viii) follows by combining conditions (v) and (vii).

(viii) $\Rightarrow$ (i) The epimorphism $F Q_{V} \rightarrow S_{1, V}$ must split and so $F Q_{V} \cong$ $S_{1, V} \oplus M$ for some Mackey functor $M$. Since $F Q_{V}$ has a unique epimorphic image we have $M=0$, and so $F Q_{V}$ is projective. We now apply (iv) $\Rightarrow$ (i).

(i) $\Leftrightarrow$ (ix) may be proved in a similar fashion, or else by using (i) $\Leftrightarrow$ (viii) together with the isomorphism $S_{1, V}^{*} \cong S_{1, V^{*}}$ and the fact that these functors are projective precisely when $S_{1, V}$ is injective.

(13.2) Corollary. $S_{H, V}$ is a projective simple Mackey functor if and only if $H$ is a p-perfect subgroup of $G$ and $V$ is a projective simple $k \bar{N}_{G}(H)$-module.

Proof. Suppose first that $S_{H, V}$ is projective and let $J=O^{p}(H)$. By 9.6 and the equivalence of categories 10.1 , we know that $S_{H, V}$ corresponds to a projective simple Mackey functor $P$ for $\bar{N}(J)$. Since $P(1) \neq 0$ by 12.2 , it follows that $S_{H, V}(J) \neq 0$ and since $H$ is a minimum subgroup of $S_{H, V}$ we deduce that $H=J$ is $p$-perfect. Since $S_{H, V}=\left(\operatorname{Inf}_{\bar{N}(H)}^{N(H)} S_{1, V}^{\bar{N}(H)} \uparrow_{N(H)}^{G}\right.$, the projective simple Mackey functor $P$ corresponding to $S_{H, V}$ is in fact $S_{1, V}^{\bar{N}(H)}$. It follows now from 13.1 that $V$ must be a projective module.

For the converse, if $H$ is a $p$-perfect subgroup and $V$ is a projective simple $k \bar{N}_{G}(H)$-module then by $13.1 S_{1, V}^{\bar{N}(H)}$ is a projective Mackey functor for $\bar{N}(H)$. The equivalence of categories 10.1 tells us that $S_{H, V}$ is a projective Mackey functor for $G$.

An extension of result 13.2 will be given at the end of Section 17.

(13.3) Theorem. Let $V$ be a simple $k G$-module. The Mackey functor projective cover and injective envelope of $S_{1, V}$ coincide, and are isomorphic to both $F P_{P_{V}}$ and $F Q_{P_{V}}$, where $P_{V}$ is the projective cover of $V$ as a $k G$-module. In particular, $P_{1, V}$ has a simple socle isomorphic to its unique simple quotient $S_{1, V}$.

Proof. We first show that $P_{1, V} \cong F P_{P_{V}}$. By adjointness, the epimorphism $F P_{P_{V}}(1)=P_{V} \rightarrow V$ extends to a morphism of Mackey functors $F P_{P_{V}} \rightarrow F P_{V}$. 
Since $F P_{P_{V}} \cong F Q_{P_{V}}$ this Mackey functor has the property that the maps $I_{H}^{K}$ are always surjective. Hence its image in $F P_{V}$ also has this property, so must be $S_{1, V}$. We thus obtain an epimorphism $F P_{P_{V}} \rightarrow S_{1, V}$. Since $F P_{P_{V}}$ is projective by 13.1, it follows that the projective cover $P_{1, V}$ is a direct summand. At the identity subgroup this projective cover is a module which has $V$ as an image and is a direct summand of $F P_{P_{V}}(1)=P_{V}$, which is indecomposable. Hence $P_{1, V}(1)=P_{V}$. But since the maps $I_{K}^{H}$ are always surjective in $F Q_{P_{V}}$, this functor is generated by its value at the identity subgroup by 2.4. Thus $P_{1, V}=F P_{P_{V}}$ as claimed.

The isomorphism of $F P_{P_{V}}$ and $F Q_{P_{V}}$ is given by 13.1. To obtain the injective envelope of $S_{1, V}$ we dualize what we have proved so far, to obtain that the injective envelope of $S_{1, V^{*}}$ is $F Q_{P_{v^{*}}}$. Interchanging $V$ and $V^{*}$ gives the injective envelope as $F Q_{P_{V}} \cong P_{1, V}$.

We may also extract from the last dualization argument the following statement.

(13.4) Corollary. $P_{1, V}^{*} \cong P_{1, V^{*}}$.

It follows from 13.3 that $P_{1, V}$ is a fixed point functor. We now emphasize that the converse holds.

(13.5) Corollary. Let $H$ be a p-group and let $U=P_{H, V}(1)$ be the trivial source module corresponding to the indecomposable projective $P_{H, V}$. If $H \neq 1$, then $P_{H, V}$ is not a fixed point functor. In particular the canonical epimorphism $P_{H, V} \rightarrow F P_{U}$ (see 12.4) is not an isomorphism.

Proof. If $P_{H, V}$ is a fixed point functor, then $P_{H, V} \rightarrow F P_{U}$ is an isomorphism and so $U$ is a projective module by 13.1 . But $U$ has vertex $H$ by 12.7 , so $H=1$.

\section{EXTENSIONS OF SIMPLE MACKEY FUNCTORS}

Throughout this section we will work over a field $k$. We present a major tool in determining the Loewy series of the projective Mackey functors, which is the calculation of the groups $\operatorname{Ext}\left(S_{H, V}, S_{K, W}\right)$. By this notation we really mean $\operatorname{Ext}_{\mu_{k}(G)}^{1}\left(S_{H, V}, S_{K, W}\right)$. The main result is 14.3 in which we show that in certain cases this information may be obtained from the functor $\left(\operatorname{Inf} F P_{W}\right) \uparrow_{N(K)}^{G}$ which has $S_{K, W}$ as its simple socle and was of importance in [20] in the construction of $S_{K, W}$. It is a practical proposition to compute with this functor since everything about it is determined by the module $W$, so in principle it is known. On the other hand, to compute the Ext groups directly from $P_{K, W}$ is generally not possible since one does not have $a$ priori sufficient information about this indecomposable projective. In the remaining cases which are not covered by $\left(\operatorname{Inf} F P_{W}\right) \uparrow_{N(K)}^{G}$ we have to consider $\left(\operatorname{Inf} F P_{P_{W}}\right) \uparrow_{N(K)}^{G}$ instead, where $P_{W}$ denotes the projective cover of $W$ as a $k \bar{N}(K)$-module.

We start with two lemmas, the first of which is the fundamental mechanism behind what is going on.

(14.1) Lemma. Let $0 \rightarrow S_{K, W} \rightarrow M \rightarrow L \rightarrow 0$ be an extension of Mackey functors in which

(a) $\operatorname{Soc}(M)=S_{K, W}$, and 
(b) $M(H)=0$ whenever $H$ is a proper subgroup of $K$.

Then $M$ can be embedded as a subfunctor of $\left(\operatorname{Inf}_{\bar{N}(K)}^{N(K)} F P_{P_{W}}\right) \uparrow_{N(K)}^{G}$, where $P_{W}$ is the projective cover of $W$ as a $k \bar{N}(K)$-module. If we suppose further that

(c) $M(K)=W$,

then $M$ can be embedded as a subfunctor of $\left(\operatorname{Inf}_{\bar{N}(K)}^{N(K)} F P_{W}\right) \uparrow_{N(K)}^{G}$.

Proof. We first assume conditions (a) and (b). Since $M$ has a simple socle it suffices to show the existence of a map $M \rightarrow\left(\operatorname{Inf}_{\bar{N}(K)}^{N(K)} F P_{P_{W}}\right) \uparrow_{N(K)}^{G}$ which is non-zero on the socle. Consider the bijections of maps

$$
\begin{aligned}
\operatorname{Hom}\left(M,\left(\operatorname{Inf}_{\bar{N}(K)}^{N(K)} F P_{P_{W}}\right) \uparrow_{N(K)}^{G}\right) & \cong \operatorname{Hom}\left(M \downarrow_{N(K)}^{G}, \operatorname{Inf}_{\bar{N}(K)}^{N(K)} F P_{P_{W}}\right) \\
& \cong \operatorname{Hom}\left(\left(M \downarrow_{N(K)}^{G}\right)^{+}, F P_{P_{W}}\right) \\
& \cong \operatorname{Hom}\left(\left(M \downarrow_{N(K)}^{G}\right)^{+}(1), P_{W}\right) \\
& \cong \operatorname{Hom}\left(\bar{M}(K), P_{W}\right) \\
& =\operatorname{Hom}\left(M(K), P_{W}\right),
\end{aligned}
$$

and note that the latter equality is a consequence of (b). To ensure that a map $M \rightarrow\left(\operatorname{Inf}_{\bar{N}(K)}^{N(K)} F P_{P_{W}}\right) \uparrow_{N(K)}^{G}$ is non-zero on $\operatorname{Soc}(M)$ it is sufficient that the corresponding map $M(K) \rightarrow P_{W}$ should have non-zero composite with the inclusion $W \rightarrow M(K)$, since the image of $W$ in $M(K)$ is $(\operatorname{Soc}(M))(K)$. (In fact this condition is also necessary.) But we can certainly find such a map since $P_{W}$ is the injective envelope of $W$. This completes the proof of the first assertion.

For the second assertion we assume the extra condition (c). The argument is the same as the one we have just given, except that we replace $P_{W}$ by $W$. At the end we need to find a map $M(K) \rightarrow W$ which has non-zero composite with $W \rightarrow M(K)$, but this is assured by condition (c).

(14.2) Lemma. Let $H \leq G$ and let $A$ be a $k \bar{N}(H)$-module whose socle is simple. Then $L=\left(\operatorname{Inf}_{\bar{N}(H)}^{N(H)} F P_{A}\right) \uparrow_{N(H)}^{G}$ has a simple socle as a Mackey functor. It is the subfunctor of $L$ generated by $\operatorname{Soc}(A) \subseteq L(H)=A$.

Proof. By definition of induction (see also [20, 4.3]), we have

$$
L(H)=\bigoplus_{g \in\left[H \backslash G / N_{G}(H)\right]}\left(\operatorname{Inf}_{\bar{N}(H)}^{N(H)} F P_{A}\right)\left(H \cap H^{g}\right)=\left(\operatorname{Inf}_{\bar{N}(H)}^{N(H)} F P_{A}\right)(H)=A .
$$

Let $N$ be a non-zero subfunctor of $L$. It suffices to show that $N(H) \supseteq \operatorname{Soc}(A)$. Since $N$ is non-zero there is a subgroup $K$ for which $N(K) \neq 0$, and we may assume $K \geq H$. For the functor $F P_{A}$ the mappings $R_{H}^{K}$ are monomorphisms. After inflation and induction it is still true for these subgroups that $R_{H}^{K}$ is a monomorphism (see [20,4.3]), so we deduce that the image $R_{H}^{K} N(K)$ is a non-zero submodule of $L(H)=A$. Therefore $N(H) \neq 0$ and we deduce the inclusion $N(H) \supseteq \operatorname{Soc}(A)$.

(14.3) Theorem. Let $S_{H, V}, S_{K, W}$ be simple Mackey functors over a field $k$.

(i) $\operatorname{Ext}\left(S_{H, V}, S_{K, W}\right)=0$ unless either $H \leq_{G} K$ or $K \leq_{G} H$. 
(ii) If $H=K$ then

$$
\begin{aligned}
\operatorname{dim} \operatorname{Ext}\left(S_{H, V}, S_{H, W}\right)= & \text { multiplicity of } S_{H, V} \\
& \text { in the second socle layer of }\left(\operatorname{Inf} F P_{P_{W}}\right) \uparrow_{N(H)}^{G} \\
= & \text { multiplicity of } S_{H, W} \\
& \text { in the second Loewy layer of }\left(\operatorname{Inf} F Q_{P_{V}}\right) \uparrow_{N(H)}^{G} .
\end{aligned}
$$

The evaluation at $H$ induces a morphism

$$
\operatorname{Ext}_{\mu_{k}(G)}\left(S_{H, V}, S_{H, W}\right) \rightarrow \operatorname{Ext}_{k \bar{N}(H)}(V, W)
$$

which is injective. In particular

$$
\operatorname{dim} \operatorname{Ext}\left(S_{H, V}, S_{H, W}\right) \leq \operatorname{dim}_{\operatorname{Ext}_{k}(H)}(V, W) .
$$

(iii) If $K<_{G} H$ then

$$
\begin{aligned}
\operatorname{dim} \operatorname{Ext}\left(S_{H, V}, S_{K, W}\right)= & \text { multiplicity of } S_{H, V} \\
& \text { in the second socle layer of }\left(\operatorname{Inf} F P_{W}\right) \uparrow_{N(K)}^{G} \\
= & \text { multiplicity of } S_{H, V^{*}} \\
& \text { in the second Loewy layer of }\left(\operatorname{Inf} F Q_{W^{*}}\right) \uparrow_{N(K)}^{G}
\end{aligned}
$$

(iv) If $H<_{G} K$ then

$$
\begin{aligned}
\operatorname{dim} \operatorname{Ext}\left(S_{H, V}, S_{K, W}\right)= & \text { multiplicity of } S_{K, W} \\
& \text { in the second Loewy layer of }\left(\operatorname{Inf} F Q_{V}\right) \uparrow_{N(H)}^{G} \\
= & \text { multiplicity of } S_{K, W^{*}} \\
& \text { in the second socle layer of }\left(\operatorname{Inf} F P_{V^{*}}\right) \uparrow_{N(H)}^{G}
\end{aligned}
$$

Proof. Suppose we have a non-split extension $0 \rightarrow S_{K, W} \rightarrow M \rightarrow S_{H, V} \rightarrow 0$ and that $H \leq_{G} K$. We will show that $K \leq_{G} H$. This Mackey functor $M$ satisfies conditions (a) and (b) of 14.1 and so $M$ embeds in $\left(\operatorname{Inf}_{\bar{N}(K)}^{N(K)} F P_{P_{W}}\right) \uparrow_{N(K)}^{G(}$. But this induced Mackey functor is only non-zero on subgroups containing $K$ (up to conjugacy) and so $K \leq_{G} H$. This proves part (i).

We will use all the time the fact that $\operatorname{dim} \operatorname{Ext}\left(S_{H, V}, S_{K, W}\right)=n$ if and only if $n$ is the largest number $r$ for which there is an extension

$$
0 \rightarrow S_{K, W} \rightarrow M \rightarrow\left(S_{H, V}\right)^{r} \rightarrow 0
$$

in which $\operatorname{Soc}(M)=S_{K, W}$.

We turn to the proof of part (ii). The statement about Loewy layers follows from the statement about socle layers by duality. Let $L=\left(\operatorname{Inf}_{\bar{N}(H)}^{N(H)} F P_{P_{W}}\right) \uparrow_{N(H)}^{G}$. By 14.1, given an extension $(*)$ then $M$ must appear as a subfunctor of $L$ and so $S_{H, V}$ appears at least $r$ times in the second socle layer of $L$. Conversely, if $S_{H, V}$ appears $r$ times in the second socle layer of $L$, then because $L$ has $S_{H, W}$ as its simple socle by 14.2 it has a subfunctor $M$ appearing in such an extension $(*)$. This proves the equality of $\operatorname{dim} \operatorname{Ext}\left(S_{H, V}, S_{H, W}\right)$ and the multiplicity of $S_{H, V}$ in the second socle layer of $L$. To show that 
$\operatorname{Ext}_{\mu_{k}(G)}\left(S_{H, V}, S_{H, W}\right) \rightarrow \operatorname{Ext}_{k \bar{N}(H)}(V, W)$ is injective, suppose we have a nonsplit extension

$$
0 \rightarrow S_{H, W} \rightarrow M \rightarrow S_{H, V} \rightarrow 0 .
$$

Evaluated at $H$ this gives a short exact sequence of $k \bar{N}(H)$-modules

$$
0 \rightarrow W \rightarrow M(H) \rightarrow V \rightarrow 0
$$

which we show is non-split. For if this sequence were to split, the splitting $M(H) \rightarrow W$ would extend uniquely to a morphism $M \rightarrow\left(\operatorname{Inf}_{\bar{N}(H)}^{N(H)} F P_{W}\right) \uparrow_{N(H)}^{G}$, using a sequence of adjunctions as in 14.1 and the fact that $\bar{M}(H)=M(H)$. The image of this morphism must be $S_{H, W}$ because the evaluation of the functor $\left(\operatorname{Inf}_{\bar{N}(H)}^{N(H)} F P_{W}\right) \uparrow_{N(H)}^{G}$ at $H$ is equal to $W$ (by an easy computation) and so $S_{H, W}$ is the only composition factor of $M$ which can appear in the image. Hence we have a morphism $M \rightarrow S_{H}, W$. It splits the original sequence since endomorphisms of $S_{H, W}$ are determined by their effect at the subgroup $H$, so the composite $S_{H, W} \rightarrow M \rightarrow S_{H, W}$ is the identity, since this is so at the level of $H$. We have thus shown that the morphism of Ext groups is injective, from which the inequality on dimensions immediately follows. Alternatively one can prove the fact that the extension $0 \rightarrow S_{H, W} \rightarrow M \rightarrow S_{H, V} \rightarrow 0$ splits if its evaluation at $H$ does by applying Proposition 3.2 of [20].

The second part of each of (iii) and (iv) follows from the first by duality. Also (iv) follows from (iii) by duality, for the reason that if we have an extension of Mackey functors $0 \rightarrow S_{K, W} \rightarrow M \rightarrow S_{H, V} \rightarrow 0$ with $H<_{G} K$ then the sequence $0 \rightarrow S_{H, V^{*}} \rightarrow M^{*} \rightarrow S_{K, W^{*}} \rightarrow 0$ is an extension to which the condition of (iii) applies. Hence it will suffice to prove the first part of (iii), and we now do this. On the one hand, if we have an extension $(*)$ then by $14.1 \mathrm{M}$ embeds as a subfunctor of $\left(\operatorname{Inf}_{\bar{N}(K)}^{N(K)} F P_{W}\right) \uparrow_{N(K)}^{G}$ and therefore $S_{H, V}$ appears at least $r$ times in the second socle layer of $\left(\operatorname{Inf}_{\bar{N}(K)}^{N(K)} F P_{W}\right) \uparrow_{N(K)}^{G}$. Conversely, if we have $r$ copies of $S_{H, V}$ in the second socle layer of $\left(\operatorname{Inf}_{\bar{N}(K)}^{N(K)} F P_{W}\right) \uparrow_{N(K)}^{G}$ then there is a subfunctor $M$ with an extension $0 \rightarrow S_{K, W} \rightarrow M \rightarrow\left(S_{H, V}\right)^{r} \rightarrow 0$, and this establishes the desired equality.

Remark. In general we may have a strict inequality in case (ii) above when $H=K$, as happens, for example, with $G$ the cyclic group of order 2 over the field of 2 elements and $H=K=1$. Here $\operatorname{Ext}\left(S_{1, k}, S_{1, k}\right)=0$ but $\operatorname{dim} \operatorname{Ext}_{k G}(k, k)=1$.

Our information about Ext groups gives another proof of the semisimplicity of $\mu_{k}(G)$ over a field $k$ in which $|G|$ is invertible, a result proved in [20].

(14.4) Corollary. If $|G|$ is invertible in the base field $k$, then $\mu_{k}(G)$ is semisimple.

Proof. It suffices to show that all Ext groups of simple Mackey functors are zero. Let $W$ be a simple $k \bar{N}_{G}(K)$-module. From the description of simple Mackey functors, we know that $S_{1, W}^{\bar{N}(K)}=F P_{W}$ and so $S_{K, W}^{G}=\left(\operatorname{Inf} F P_{W}\right) \uparrow_{N(K)}^{G}$. Also $W=P_{W}$ since $k \bar{N}_{G}(K)$ is semisimple. Therefore all Mackey functors appearing in 14.3 are simple, and in particular their second socle layers are zero. 
We will now improve on Theorem 14.3, but first we need a lemma.

(14.5) Lemma. For a Mackey functor $M$ the following are equivalent:

(i) $M$ is $H$-projective.

(ii) Every morphism $N \rightarrow M$ which is split epi on restriction to $H$ is split epi.

(iii) every morphism $M \rightarrow N$ which is split mono on restriction to $H$ is split mono.

Proof. We prove that (i) implies (ii). Suppose that $M$ is $H$-projective, and let $\alpha: N \rightarrow M$ be a morphism such that its restriction $N \downarrow_{H}^{G} \rightarrow M \downarrow_{H}^{G}$ is split epi, split by a morphism $\beta: M \downarrow_{H}^{G} \rightarrow N \downarrow_{H}^{G}$. Then $\beta \uparrow_{H}^{G}$ splits the top arrow in the following commutative square:

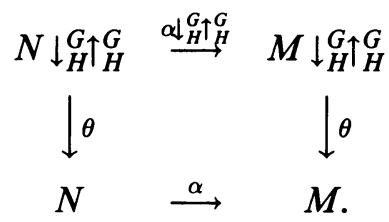

Since $M$ is supposed to be $H$-projective, $\theta: M \downarrow_{H}^{G} \uparrow_{H}^{G} \rightarrow M$ is split by a map $\eta: M \rightarrow M \downarrow_{H}^{G} \uparrow_{H}^{G}$, and we claim that $\theta \beta \uparrow_{H}^{G} \eta$ splits $\alpha$. We verify

$$
\alpha \theta \beta \uparrow_{H}^{G} \eta=\theta \alpha \downarrow_{H}^{G} \uparrow_{H}^{G} \beta \uparrow_{H}^{G} \eta=\theta \eta=1 .
$$

The argument that (ii) implies (i) is straightforward, since the natural transformation $M \downarrow_{H}^{G \dagger} \uparrow_{H}^{G} \rightarrow M$ is always split epi on restriction to $H$.

The equivalence of (i) and (iii) is similar.

Recall that by 11.4 , a vertex $\mathrm{vx}\left(S_{H, V}\right)$ of $S_{H, V}$ satisfies $H \triangleleft \mathrm{vx}\left(S_{H, V}\right)$ and $\operatorname{vx}\left(S_{H, V}\right) / H=\operatorname{vx}(V)$.

(14.6) Theorem. If $\operatorname{Ext}\left(S_{H, V}, S_{K, W}\right) \neq 0$ then either

$$
H \leq_{G} K \leq_{G} \mathbf{v x}\left(S_{H, V}\right)
$$

or

$$
K \leq_{G} H \leq_{G} \operatorname{vx}\left(S_{K, W}\right) .
$$

In particular there is an element $g \in G$ so that either $H \triangleleft{ }^{g} K$ or $K \triangleleft^{g} H$.

Proof. We already know that to have a non-split extension we must have either $H \leq_{G} K$ or $K \leq_{G} H$. Suppose that $H \leq_{G} K$ and that

$$
0 \rightarrow S_{K, W} \rightarrow M \rightarrow S_{H, V} \rightarrow 0
$$

is a non-split extension. We show that $K \leq_{G} \operatorname{vx}\left(S_{H, V}\right)$. If not, then the restriction of the sequence to $\operatorname{vx}\left(S_{H, V}\right)$ is the sequence

$$
0 \rightarrow 0 \rightarrow M \downarrow_{\mathrm{vx}\left(S_{H, V}\right)} \rightarrow S_{H, V} \downarrow_{\mathrm{vx}\left(S_{H, V}\right)} \rightarrow 0
$$

so that evidently the epimorphism shown here is split. Therefore by the last lemma the map $M \rightarrow S_{H, V}$ must be split epi, which is a contradiction. Therefore $K \leq_{G} \mathrm{vx}\left(S_{H, V}\right)$.

The argument when $K \leq_{G} H$ is similar. 
(14.7) Corollary. If $(H, V)$ is a weight, and if $\operatorname{Ext}\left(S_{H, V}, S_{K, W}\right) \neq 0$ then $K<_{G} H$.

Proof. If $K \geq_{G} H$ then by $14.6, K={ }_{G} H$ since $\operatorname{vx}\left(S_{H, V}\right)=H$. But by 14.3 we have $\operatorname{Ext}\left(S_{H, V}, S_{K, W}\right)=0$ if $K=H$ because its dimension is bounded by $\operatorname{dim} \operatorname{Ext}_{k \bar{N}(H)}(V, W)$ which is zero since $V$ is projective. The only remaining possibility allowed by 14.3 is $K<_{G} H$.

\section{THE COMPUTATION OF SUBFUNCTOR LATTICES}

We illustrate the techniques which we have so far assembled by using them to give the complete lattice of subfunctors of certain Mackey functors. In order to have non-split extensions we will work always over a field $k$ of characteristic $p$. As our examples, we will mostly take the fixed point functors $F P_{V}$, which we have seen play a fundamental role in the determination of Mackey functor structure. We now start with the most basic of these, namely $F P_{k}$.

(15.1) Lemma. Let $k$ be a field of characteristic $p$, and let $H$ be a p-subgroup of $G$. Then $S_{H, k}(K)=0$ unless some conjugate of $H$ is a Sylow p-subgroup of $K$, in which case $S_{H, k}(K)=k$.

Proof. First notice that $S_{1, k}(K)=\left(\sum_{x \in K} x\right) \cdot k=|K| \cdot k$, and this in non-zero precisely if $p \nmid|K|$. Now

$$
\begin{aligned}
S_{H, k}^{G}(K) & =\left(\operatorname{Inf}_{\bar{N}(H)}^{N(H)} S_{1, k}^{\bar{N}(H)}\right) \uparrow_{N(H)}^{G}(K) \\
& =\bigoplus_{x \in[N(H) \backslash G / K]}\left(\operatorname{Inf}_{\bar{N}(H)}^{N(H)} S_{1, k}^{\bar{N}(H)}\right)\left(N(H) \cap{ }^{x} K\right)
\end{aligned}
$$

and the only non-zero terms occur when ${ }^{x} K \supseteq H$, by the definition of inflation. Furthermore, $p$ must not divide $\left|\left(N_{G}(H) \cap{ }^{x} \bar{K}\right): H\right|=\left|N_{x_{K}}(H): H\right|$. By a wellknown property of $p$-groups this happens precisely if $H$ is a Sylow $p$-subgroup of ${ }^{x} K$. Equivalently, $H^{x}$ must be a Sylow $p$-subgroup of $K$. We complete the argument by showing that there is at most one double coset of elements $x$ for which this happens. For suppose $H^{x}$ and $H^{y}$ are both Sylow $p$-subgroups of $K$. Then $H^{x k}=H^{y}$ for some $k \in K$. Now $x k y^{-1}=n \in N_{G}(H)$, so $y=n^{-1} x k \in N_{G}(H) x K$.

We work with the map $\psi$ constructed in Section 6, and we wish to refer to its matrix taken with respect to the two bases $\left\{S_{H, V}\right\}$ and $\left\{\sigma_{H, V}\right\}$ of its domain and codomain. We denote this matrix by $\Psi$. The columns of $\Psi$ are given by the evaluations $S_{H, V}(K) \in G_{0}\left(k \bar{N}_{G}(K)\right)$ and may be computed using the explicit formula 8.8 for the simple Mackey functors. Several examples are given in Section 21.

(15.2) Corollary. Let $k$ be a field of characteristic $p$. The submatrix of $\Psi$ with rows and columns corresponding to basis elements $S_{H, k}$ and $\sigma_{H, k}$ where $H$ is a p-group, is the identity matrix.

(15.3) Corollary. Let $k$ be a field of characteristic $p$.

(i) The composition factors of the Mackey functor $F P_{k}$ are the simple functors $S_{H, k}$, one for each conjugacy class of p-subgroups $H$. These are also the composition factors of $F Q_{k}$. 
(ii) The Mackey subfunctors $L$ of $F P_{k}$ are in bijection with the sets $\mathscr{X}$ of p-subgroups of $G$ closed under conjugation and taking subgroups. The bijection is given as follows: if $L \subseteq F P_{k}$ we associate

$$
\mathscr{X}=\{H \leq G \mid H \text { is a p-subgroup, } L(H) \neq 0\},
$$

and if $\mathscr{Z}$ is a set of p-subgroups closed under conjugation and taking subgroups we associate the subfunctor

$$
L=\left\langle F P_{k}(H) \mid H \in \mathscr{X}\right\rangle .
$$

Proof. (i) We have

$$
\psi\left(F P_{k}\right)=\sum_{\substack{H \\ \text { up to conjugacy }}} \sigma_{H, k}=\sum_{\substack{H \text { a p-roup } \\ \text { uptoconjugecy }}} \psi\left(S_{H, k}\right),
$$

using 15.1. The result for $F P_{k}$ follows from the fact that $\psi$ is an isomorphism. Exactly the same argument works for $F Q_{k}$, but the composition factors may also be deduced from those of $F P_{k}$ by duality.

(ii) Let us denote the mappings described in the statement by

$$
\begin{aligned}
& \alpha: L \mapsto\{H \leq G \mid H \text { is a } p \text {-subgroup, } L(H) \neq 0\} \\
& \beta: \mathscr{X} \mapsto\left\langle F P_{k}(H) \mid H \in \mathscr{X}\right\rangle .
\end{aligned}
$$

It is evident that $\alpha(L)$ is closed under conjugation, and if $K \leq H \in \alpha(L)$ then since $R_{K}^{H}$ is the identity on $F P_{k}(H)$ we have $L(K) \neq 0$, so $K \in \alpha(L)$. We need not check anything like this in the definition of $\beta$, since $\beta(\mathscr{Z})$ is by definition a subfunctor of $F P_{k}$.

To check that $\alpha$ and $\beta$ are mutually inverse, observe that $\beta \alpha(N)$ is a subfunctor of $N$, and it has at least as many composition factors as $N$ since by our previous computations for $F P_{k}$ there is one for each $p$-subgroup $H$ with $\beta \alpha(N)(H) \neq 0$. Hence $\beta \alpha(N)=N$.

On the other hand, given a family $\mathscr{X}$ of $p$-subgroups of $G$, if $H$ is a $p$ subgroup then $\beta(\mathscr{X})(H) \neq 0$ if and only if $H \in \mathscr{X}$. This follows from the formula 2.4 , namely

$$
\beta(\mathscr{Q})(H)=\sum_{\substack{K \in \mathscr{Z} \\ K \leq H}} I_{K}^{H} F P_{k}(K),
$$

and the fact that if $K$ and $H$ are $p$-groups then $I_{K}^{H}=0$ unless $K=H$. Thus $\alpha \beta(\mathscr{X})=\mathscr{X}$.

We may express 15.3 (ii) by saying that $F P_{k}$ has a diagram (in the sense of Alperin [1]) whose vertices are the conjugacy classes of $p$-subgroups of $G$, and where one conjugacy class with representative $K$ is placed below another with representative $H$ if $K$ is conjugate to a subgroup of $H$.

A second example where we work out the full lattice of subfunctors is given at the beginning of Section 20, where we treat the indecomposable projective Mackey functors in the situation where $G$ has a normal Sylow $p$-subgroup of order $p$.

As we have already seen, we may find the composition factors of any Mackey functor by computing the matrix $\Psi$, but as far as deciding how the composition 
factors should appear in the lattice of subfunctors we need to employ a further technique. To assist in this we bring in the bar construction

$$
\bar{M}(H)=M(H) / \sum_{K<H} I_{K}^{H} M(K),
$$

which was of use in Section 6 in obtaining a sufficient condition for a simple Mackey functor to appear as a composition factor of a Mackey functor $M$, and in obtaining complete information in case $M$ is completely reducible. We now refine this to obtain a necessary and sufficient condition that the simple Mackey functor should appear in the top of an arbitrary Mackey functor $M$, that is that it should be a homomorphic image of $M$. Since any non-zero map to a simple object is necessarily an epimorphism we are thus interested in the simple Mackey functors for which there exists a non-zero morphism $M \rightarrow S_{H, W}$.

Recall that if $H \leq K$ and $M$ is a Mackey functor for $K$, we have the Brauer morphism which is the composite

$$
\beta_{H}: M(K) \stackrel{R_{H}^{K}}{\longrightarrow} M(H) \rightarrow \bar{M}(H) .
$$

(15.4) Proposition. There is a non-zero homomorphism $M \rightarrow S_{H, U}$ if and only if

(i) there is an epimorphism of $\bar{N}_{G}(H)$-modules $\alpha: \bar{M}(H) \rightarrow U$, and

(ii) for all subgroups $K$ with $H \leq K \leq N_{G}(H)$ the composite

$$
M(K) \stackrel{\beta_{H}}{\longrightarrow} \bar{M}(H) \stackrel{\alpha}{\longrightarrow} U
$$

has image contained in $\left(\sum_{g \in[K / H]} g\right) \cdot U$.

Proof. There is a non-zero map $M \rightarrow S_{H, U}$ if and only if there is a nonzero map of Mackey functors for $G, M \rightarrow\left(\operatorname{Inf}_{\bar{N}_{G}(H)}^{N_{G}(H)} F P_{U}\right) \uparrow_{N_{G}(H)}^{G}$, whose image lies in the socle of $\left(\operatorname{Inf}_{\bar{N}_{G}(H)}^{N_{G}(H)} F P_{U}\right) \uparrow_{N_{G}(H)}^{G}$. By adjointness of induction and the description of the socle [20, 8.1], this happens if and only if there is a non-zero map of Mackey functors for $N_{G}(H), M \rightarrow \operatorname{Inf}_{\bar{N}_{G}(H)}^{N_{G}(H)} F P_{U}$, whose image lies in the socle of $\operatorname{Inf}_{\bar{N}_{G}(H)} N_{N_{G}(H)} F P_{U}$. Again by adjointness this is equivalent to requiring a non-zero map $M^{+} \rightarrow F P_{U}$ whose image is contained in $\operatorname{Soc}\left(F P_{U}\right)$. At the identity subgroup $H / H$ of $\bar{N}_{G}(H)$, such a map gives rise to an epimorphism $\alpha: M^{+}(H / H)=\bar{M}(H) \rightarrow U$, which is condition (i). At an arbitrary subgroup $K / H \leq \bar{N}_{G}(H)$ the map is the composite $M^{+}(K / H) \stackrel{R}{\longrightarrow} M^{+}(H / H) \stackrel{\alpha}{\longrightarrow} U$, by $[20,6.1]$. Our requirement is that its image is contained in $\left(\operatorname{Soc}\left(F P_{U}\right)\right)(K / H)$, which equals $\left(\sum_{g \in[K / H]} g\right) \cdot U$ by $[20,7.1]$, This is equivalent to our condition (ii).

In the next corollaries we apply 15.4 in the situation where $M=F P_{V}$ and $V$ is a $k G$-module. The extra condition we require in 15.5 , that $V^{H}$ be completely reducible as a $k \bar{N}_{G}(H)$-module, seems often to be satisfied in practice, and it allows us to restrict the possibilities for the top Loewy layer of $F P_{V}$. It also indicates that simple Mackey functors parametrized by weights tend to occur there. 
(15.5) Corollary. If there exists a non-zero homomorphism $F P_{V} \rightarrow S_{H, U}$ and if $V^{H}$ is a direct sum of simple $k \bar{N}_{G}(H)$-modules then $U$ must be a projective $k \bar{N}_{G}(H)$-module.

Proof. Since $F P_{V}(H)$ and hence $\overline{F P_{V}}(H)$ are completely reducible, the epimorphism $\alpha: \overline{F P_{V}}(H) \rightarrow U$ must arise from an isomorphism between an irreducible summand of $V^{H}$ and $U$. This therefore induces for each $K$ an isomorphism between a summand of $V^{K}$ and $U^{K}$, so that the composite mapping in 15.4(ii) must be an epimorphism onto $U^{K}$. The image of this map is contained in $\left(\sum_{g \in[K / H]} g\right) \cdot U$ by 15.4 , so we deduce that

$$
\left(\sum_{g \in[K / H]} g\right) \cdot U=U^{K}
$$

for all subgroups $K$ with $H \leq K \leq N_{G}(H)$. This means that the $k \bar{N}_{G}(H)$ module $U$ has the property that the trace from the identity is always surjective onto fixed points under arbitrary subgroups. Such a module is necessarily projective.

(15.6) Corollary. If $\operatorname{dim} V=1$ then $F P_{V}$ has $S_{Q, V \downarrow_{N(Q)}}$ as its unique simple image, where $Q$ is a Sylow p-subgroup of $G$.

Proof. Certainly there exists some simple Mackey functor $S_{H, U}$ which is an image of $F P_{V}$. The last corollary applies since $V^{H}$ is simple, so we require a pair $(H, U)$ where $H$ is a $p$-subgroup, $\operatorname{dim} U=1$ and $U$ is projective as a module for $\bar{N}_{G}(H)$. The only possibility is that $H=Q$ is a Sylow $p$-subgroup since otherwise $p|| \bar{N}_{G}(H) \mid$ and $U$ could not be projective. It follows that $U \cong V^{Q}=V \downarrow_{N(Q)}$.

The necessary and sufficient condition of 15.4 is not always easy to verify in practice, and so we now develop this into a further condition in 15.7(ii) which guarantees that certain simple Mackey functors will appear as quotients of $M$.

(15.7) Proposition. Let $M$ be a Mackey functor, and let $H$ be a maximal subgroup such that $\bar{M}(H) \neq 0$.

(i) If $H \leq K \leq G$, the image of the Brauer morphism $\beta_{H}: M(K) \rightarrow \bar{M}(H)$ is equal to $\left(\sum_{g \in\left[N_{K}(H) / H\right]} g\right) \cdot \bar{M}(H)$.

(ii) If $U$ is any simple homomorphic image of $\bar{M}(H)$, there exists a nonzero homomorphism $M \rightarrow S_{H, U}$.

Proof. (i) If $H=K$ the result is self-evident, so suppose that $H<K$. In this case since $\bar{M}(K)=0$ we have

$$
\begin{aligned}
M(K) & =\sum_{J<K} I_{J}^{K} M(J) \\
& =\sum_{H \leq J<K} I_{J}^{K} M(J)+\sum_{H \notin J<K} I_{J}^{K} M(J) .
\end{aligned}
$$

If for a subgroup $J, H<J<K$, then similarly $M(J)=\sum_{L<J} I_{L}^{J} M(L)$, and on substituting such an expression into the first summand above we remove all 
instances of that subgroup $J$. Eliminating such subgroups $J$ by this means we obtain

$$
M(K)=I_{H}^{K} M(H)+\sum_{H \not J<K} I_{J}^{K} M(J) .
$$

Now observe that $\beta_{K}$ is zero on the second summand here, since it gives the image in $\bar{M}(H)$ of $\sum_{H \not J<K} R_{H}^{K} I_{J}^{K} M(J)$, in which after applying the Mackey decomposition formula every term is the image of $\sum_{L<H} I_{L}^{H}$. We deduce that

$$
\begin{aligned}
\beta_{H} M(K) & =\beta_{H} I_{H}^{K} M(H) \\
= & \left(R_{H}^{K} I_{H}^{K} M(H)+\sum_{L<H} I_{L}^{H} M(L)\right) / \sum_{L<H} I_{L}^{H} M(L) \\
= & \left(\sum_{g \in[H \backslash K / H]} I_{H g \cap H}^{H} c_{g} R_{H \cap 8 H}^{H} M(H)+\sum_{L<H} I_{L}^{H} M(L)\right) / \sum_{L<H} I_{L}^{H} M(L) \\
= & \left(\sum_{g \in\left[N_{K}(H) / H\right]} I_{H}^{H} c_{g} R_{H}^{H} M(H)+\sum_{L<H} I_{L}^{H} M(L)\right) / \sum_{L<H} I_{L}^{H} M(L) \\
= & \left(\sum_{g \in\left[N_{K}(H) / H\right]} g\right) \cdot \bar{M}(H) .
\end{aligned}
$$

(ii) We need only verify condition (ii) of 15.4. This follows from part (i) in view of the fact that the epimorphism $\alpha$ which appears in 15.4 is a module morphism and so carries $\left(\sum_{g \in[K / H]} g\right) \cdot \bar{M}(H)$ into $\left(\sum_{g \in[K / H]} g\right) \cdot U$.

(15.8) Example. Let $G=G L(3,2)$ and let $R$ be the field with 2 elements. $G$ has four simple modules over $R$, namely the trivial representation, the natural 3-dimensional module, its dual, and the Steinberg module of dimension 8 . We denote these modules by $1,3,3^{*}$ and 8 . The subfunctor structure of $F_{3} P_{3}$ has a diagram (in the sense of Alperin [1])

$$
\begin{array}{|lll|}
\hline & S_{V_{2}, 2} & \\
& S_{C_{2}, 1} & \\
S_{C_{4}, 1} & & S_{V_{1}, 1} \\
& S_{1,3} & \\
\hline
\end{array}
$$

Proof. Our notation for the 2-subgroups of $G$ is that we may take

$$
C_{2}=\left\langle\left(\begin{array}{lll}
1 & 0 & 1 \\
0 & 1 & 0 \\
0 & 0 & 1
\end{array}\right)\right\rangle, \quad C_{4}=\left\langle\left(\begin{array}{lll}
1 & 1 & 0 \\
0 & 1 & 1 \\
0 & 0 & 1
\end{array}\right)\right\rangle
$$

and $V_{1}, V_{2}$ to be representatives of the two conjugacy classes of subgroups $C_{2} \times C_{2}$, which we may take to be

$$
V_{1}=\left(\begin{array}{lll}
1 & 0 & * \\
0 & 1 & * \\
0 & 0 & 1
\end{array}\right), \quad V_{2}=\left(\begin{array}{lll}
1 & * & * \\
0 & 1 & 0 \\
0 & 0 & 1
\end{array}\right)
$$


It is straightforward to compute the structure of $F P_{3}(H)$ and $\overline{F P}_{3}(H)$ :

\begin{tabular}{|c|c|c|}
\hline 2-subgroup $H$ & $\operatorname{dim} F P_{3}(H)$ & $\operatorname{dim} \overline{F P}_{3}(H)$ \\
\hline 1 & 3 & 3 \\
$C_{2}$ & 2 & 1 \\
$C_{4}$ & 1 & 0 \\
$V_{1}$ & 1 & 0 \\
$V_{2}$ & 2 & 2 \\
$D_{8}$ & 1 & 0 \\
\hline
\end{tabular}

One computes also that the action of $N\left(V_{2}\right) / V_{2} \cong S_{3}$ on $F P_{3}\left(V_{2}\right)$ is as the projective simple 2-dimensional module for $S_{3}$. We suppose that we have computed the matrix $\Psi$, which is presented in Section 21. Using the method of Section 6 we conclude that $F P_{3}$ has composition factors $S_{1,3}, S_{C_{2}, 1}, S_{C_{4}, 1}, S_{V_{1}, 1}$, $S_{V_{2}, 2}$. Note here that all the composition factors must necessarily be of the form $S_{H, V}$ with $H$ a $p$-group, by Section 9.

We now look for the semisimple quotient of $F P_{3}$. The composition factor $S_{V_{2}, 2}$ must certainly appear in this quotient since the condition of 15.7 is satisfied, but this is not sufficient to eliminate the possibility that some of the other composition factors might be in this quotient as well. We eliminate $S_{C_{4}, 1}$ and $S_{V_{1}, 1}$ by 15.5. Finally we show that $S_{C_{2}, 1}$ is not a quotient of $F P_{3}$, and we suppose to the contrary that there is an epimorphism $F P_{3} \rightarrow S_{C_{2}, 1}$. In that case by 15.4 the epimorphism $F P_{3}\left(C_{2}\right) \rightarrow \overline{F P}_{3}\left(C_{2}\right)=k$ has the property that the Brauer morphism $F P_{3}\left(V_{2}\right) \rightarrow \overline{F P}_{3}\left(C_{2}\right)$ has image contained in $\left(\sum_{g \in\left[V_{2} / C_{2}\right]} g\right) \cdot k=0$. But in fact $F P_{3}\left(V_{2}\right)=F P_{3}\left(C_{2}\right)$ and the Brauer morphism is surjective, so the epimorphism cannot have this property. This shows that $F P_{3}$ has a unique maximal subfunctor, and the largest semisimple quotient of $F P_{3}$ is $S_{V_{2}, 2}$.

Let $M_{1}$ denote the unique maximal subfunctor of $F P_{3}$. We will show that $M_{1}$ in turn has a unique maximal subfunctor $M_{2}$, and that the quotient is $S_{C_{2}, 1}$. To demonstrate this we show that $S_{C_{4}, 1}$ and $S_{V_{1}, 1}$ are not images of $M_{1}$. Suppose to the contrary that $S_{C_{4}, 1}$ is an image of $M_{1}$. Then $S_{C_{4}, 1} \downarrow_{C_{4}}^{G}$ would be an image of $M_{1} \downarrow_{C_{4}}^{G}$, which equals $F P_{3} \downarrow_{C_{4}}^{G}$ since the extra composition factor $S_{V_{2}, 2}$ restricts to zero on $C_{4}$. But $F P_{3} \downarrow_{C_{4}}^{G}$ is just $F P_{3}$ when we regard the 3-dimensional module as a module for $C_{4}$, and this cannot have $S_{C_{4}, 1} \downarrow_{C_{4}}^{G}$ as an image since $\overline{F P}_{3}\left(C_{4}\right)=0$ by 15.4 . The argument which shows that $S_{V_{1}, 1}$ is not an image of $M_{1}$ is just the same, and we conclude that $S_{C_{2}, 1}$ is the unique simple image.

To complete the diagram which represents the subfunctor lattice of $F P_{3}$ we use the fact that $S_{1,3}$ is the socle of $F P_{3}$ and that there is no non-split extension of $S_{C_{4}, 1}$ and $S_{V_{1}, 1}$. This is a consequence of 14.3(i).

\section{6. СоHomological Mackey functors}

A Mackey functor for $G$ is called cohomological if whenever $K \leq H \leq G$, one has $I_{K}^{H} R_{K}^{H}=|H: K|$, that is, multiplication by the index of $K$ in $H$. These Mackey functors take their name because the restriction and corestriction of group cohomology satisfy this relationship. Our aim in this section is to show 
that all cohomological Mackey functors are closely related to group cohomology in that they are precisely the Mackey functors which are quotients of fixed point functors. In the process we determine the simple cohomological Mackey functors and describe their projective covers when the ground ring is a field.

We start this section with some elementary remarks. The cohomological Mackey functors form a full subcategory of $\operatorname{Mack}_{R}(G)$, which we denote $\operatorname{Comack}_{R}(G)$. They may be regarded as the representations of a quotient of the Mackey algebra obtained by factoring out from $\mu_{R}(G)$ the ideal generated by the elements $I_{K}^{H} R_{K}^{H}-|H: K| I_{H}^{H}$. An interesting description of the cohomological Mackey functors which is due to Yoshida [24] is that they may be identified with the additive functors

$$
F: \mathscr{H}_{G} \rightarrow R \text {-mod }
$$

where $\mathscr{H}_{G}$ is the full subcategory of $R G$-mod whose objects are the permutation modules over $R G$. Given such a functor one obtains a Mackey functor by composing with the functor

$$
G \text {-set } \rightarrow \mathscr{K}_{G}
$$

which takes the $G$-set $X$ to the permutation module $R X$. This gives the covariant part of a Mackey functor, and one obtains the contravariant part by defining $R_{K}^{H}$ to be $F$ applied to the relative trace map $R[G / H] \rightarrow R[G / K]$ where $K \leq H$.

The following two lemmas are elementary.

(16.1) Lemma. Any subfunctor and any quotient functor of a cohomological Mackey functor is cohomological.

One consequence of this result is that the simple objects in $\operatorname{Comack}_{R}(G)$ are also simple in $\operatorname{Mack}_{R}(G)$, so they are a subset of the $S_{H, V}$.

(16.2) Lemma. If $V$ is an $R G$-module, both $F P_{V}$ and $F Q_{V}$ are cohomological Mackey functors.

Another useful fact is the following characterisation of cohomological Mackey functors, which has been pointed out to us by Lluis Puig [private communication]. Note that Yoshida proves a related (but different) result [24, 4.4].

(16.3) Proposition. Let $F P_{R}$ be the fixed point functor corresponding to the trivial $R G$-module $R$, viewed as a Green functor. A Mackey functor $M$ is cohomological if and only if it is a module (in the sense of Mackey functors) over the Green functor $F P_{R}$.

Proof. Since $F P_{R}$ is cohomological, it is clear that so is any module $M$ over $F P_{R}$. Indeed if $x \in M(H)$,

$$
\begin{aligned}
I_{K}^{H} R_{K}^{H}(x) & =I_{K}^{H} R_{K}^{H}\left(1_{H} \cdot x\right)=I_{K}^{H}\left(R_{K}^{H}\left(1_{H}\right) \cdot R_{K}^{H}(x)\right) \\
& =I_{K}^{H} R_{K}^{H}\left(1_{H}\right) \cdot x=|H: K| \cdot 1_{H} \cdot x=|H: K| \cdot x .
\end{aligned}
$$

Conversely assume that $M$ is cohomological. Since $F P_{R}(H)=R$, there is a unique way to define an $F P_{R}(H)$-module structure over $M(H)$. For $\lambda \in R$, $x \in M(H)$ and $y \in M(K)$, it is plain that we have

$$
\begin{aligned}
R_{K}^{H}(\lambda \cdot x) & =R_{K}^{H}(\lambda) \cdot R_{K}^{H}(x), \\
g(\lambda \cdot x) & =g_{\lambda} \cdot g_{x}, \\
I_{K}^{H}\left(R_{K}^{H}(\lambda) \cdot y\right) & =\lambda \cdot I_{K}^{H}(y),
\end{aligned}
$$


because $R_{K}^{H}(\lambda)=\lambda$ in the functor $F P_{R}$. Finally our assumption implies that the last property in the definition of a module over $F P_{R}$ is satisfied:

$$
I_{K}^{H}\left(\lambda \cdot R_{K}^{H}(x)\right)=I_{K}^{H} R_{K}^{H}(\lambda \cdot x)=|H: K| \lambda \cdot x=I_{K}^{H}(\lambda) \cdot x .
$$

This completes the proof.

We have seen in Section 8 that in the case of arbitrary Mackey functors, the projective objects are obtained as direct summands of induced Burnside functors. We now show that the same result holds for cohomological Mackey functors, replacing the Burnside functor by the fixed point functor $F P_{R}$. As a first step we establish the result analogous to 8.2, and give a direct argument since this is quite short. We should note also that this result is immediate from the work of Yoshida [24], using Yoneda's lemma, since the functors $F P_{R \uparrow_{H}^{G}}$ are the representable functors on Yoshida's category $\mathscr{H}_{G}$. We leave to the reader the task of expressing the result as an adjointness property, as in 8.2.

(16.4) Lemma. Let $M$ be a cohomological Mackey functor.

(i) Let $m \in M(G)$. Then there exists a unique morphism $f: F P_{R} \rightarrow M$ whose evaluation at $G$ maps $1_{R}$ to $m$.

(ii) Let $H \leq G$ and $m \in M(H)$. Then there exists a unique morphism $f: F P_{R \dagger_{H}^{G}} \rightarrow M$ whose evaluation at $H$ maps $1 \otimes 1_{R}$ to $m$. (Here $\left.1 \otimes 1_{R} \in\left(R G \otimes_{R H} R\right)^{H}\right)$.

Proof. (i) There is no choice for the definition of $f$. Since $R_{H}^{G}\left(1_{R}\right)=1_{R}$ in $F P_{R}$, we have to define the evaluation of $f$ at $H$ by

$$
f(H)\left(1_{R}\right)=R_{H}^{G}(m)
$$

and extend $R$-linearly. Now we have to check that $f$ is a morphism of Mackey functors. Clearly $f$ commutes with restriction and conjugation, so we have to consider only the induction maps $I_{K}^{H}$. But this follows immediately from the fact that $M$ is cohomological:

$$
\begin{aligned}
I_{K}^{H}\left(f(K)\left(1_{R}\right)\right) & =I_{K}^{H} R_{K}^{H}\left(f(H)\left(1_{R}\right)\right)=|H: K| \cdot f(H)\left(1_{R}\right) \\
& =f(H)\left(|H: K| \cdot 1_{R}\right)=f(H)\left(I_{K}^{H}\left(1_{R}\right)\right) .
\end{aligned}
$$

(ii) By part (i), there is a unique morphism $f^{\prime}: F P_{R} \rightarrow M \downarrow_{H}^{G}$ whose evaluation at $H$ maps $1_{R}$ to $m$. By adjunction, this corresponds to a morphism $\left(F P_{R}\right) \uparrow_{H}^{G} \rightarrow M$, hence to a morphism $f: F P_{R \uparrow_{H}^{G}} \rightarrow M$ using the isomorphism given in 5.2. If one traces the effect of this isomorphism and of the adjunction, one can check easily that the evaluation of $f$ at $H$ maps $1 \otimes 1_{R}$ to $m$. Moreover the uniqueness of $f^{\prime}$ implies the uniqueness of $f$ with this property.

\section{(16.5) Theorem.}

(i) A Mackey functor $M$ for $G$ over $R$ is cohomological if and only if it is isomorphic to a quotient of a fixed point functor $F P_{V}$, where $V$ is an $R G$-module which can be chosen to be a permutation module.

(ii) $A$ cohomological Mackey functor is projective in $\operatorname{Comack}_{R}(G)$ if and only if it is isomorphic to a fixed point functor $F P_{V}$ where $V$ is a permutation-projective module for $G$ (i.e., a direct summand of a permutation module). 
Proof. (i) By 16.1 and 16.2, we already know that quotients of fixed point functors are cohomological. Conversely suppose that $M$ is cohomological. We choose a set $\left(m_{i}\right)_{i \in I}$ of Mackey functor generators of $M$, such that $m_{i} \in M\left(H_{i}\right)$ for some subgroup $H_{i}$. By 16.4, there is a morphism $f_{i}: F P_{V_{i}} \rightarrow M$ having $m_{i}$ in its image, where $V_{i}=R \uparrow_{H_{i}}^{G}$. Therefore if we set $V=\bigoplus_{i} V_{i}$, then

$$
\bigoplus_{i} f_{i}: \bigoplus_{i} F P_{V_{i}}=F P_{V} \rightarrow M
$$

is an epimorphism. This proves the result since $V$ is a permutation module.

(ii) We first prove that $F P_{R T_{H}^{G}}$ is projective. Let $\pi: M \rightarrow F P_{R T_{H}^{G}}$ be an epimorphism, where $M$ is cohomological. Let $m \in M(H)$ such that $\pi(H)(m)=1 \otimes 1_{R}$. By 16.4, there is a unique $f: F P_{R T_{H}^{G}} \rightarrow M$ such that $f(H)\left(1 \otimes 1_{R}\right)=m$. Then $\pi f: F P_{R T_{H}^{G}} \rightarrow F P_{R T_{H}^{G}}$ is the unique morphism whose evaluation at $H$ maps $1 \otimes 1_{R}$ to itself. Therefore $\pi f=\mathrm{id}$, completing the proof of the projectivity of $F P_{R T_{H}^{G}}$.

Since any permutation-projective module $V$ is a direct summand of a direct sum of modules of the form $R \uparrow_{H}^{G}$, the same holds with the corresponding fixed point functors and we obtain that $F P_{V}$ is projective.

Conversely let $P$ be a projective cohomological Mackey functor. By (i) there exists an epimorphism $F P_{V} \rightarrow P$ where $V$ is a permutation module. This splits since $P$ is projective. By 2.3 this immediately implies that $P \cong F P_{W}$ for some permutation-projective module $W$.

(16.6) Corollary. If $H$ is a subgroup of $G$ and if $M$ is a cohomological Mackey functor for $H$, then $M \uparrow_{H}^{G}$ is cohomological.

Proof. Write $M$ as a quotient of $F P_{V}$ and induce. Since induction is exact, $M \uparrow_{H}^{G}$ is a quotient of $F P_{V} \uparrow_{H}^{G} \cong F P_{V \uparrow_{H}^{G}}$.

The spirit of the following result is completely opposite to that of group cohomology.

(16.7) Corollary. Let $V$ be an RG-module. Then there exists a resolution

$$
\cdots \rightarrow V_{n} \rightarrow \cdots \rightarrow V_{0} \rightarrow V \rightarrow 0
$$

where each $V_{i}$ is a permutation module, such that the sequence of fixed points

$$
\cdots \rightarrow V_{n}^{H} \rightarrow \cdots \rightarrow V_{0}^{H} \rightarrow V^{H} \rightarrow 0
$$

is exact for every subgroup $H$ of $G$.

Proof. This is just a reformulation of the existence of a projective resolution in $\operatorname{Comack}_{R}(G)$ for the functor $F P_{V}$.

Assume now that our base ring is a field $R=k$. We wish to describe the simple cohomological Mackey functors and their projective covers. First we consider the case of characteristic zero.

(16.8) Proposition. Let $S_{H, V}$ be a simple Mackey functor for $G$ over a field $k$ whose characteristic is zero or prime to $|G|$. Then $S_{H, V}$ is cohomological if and only if $H=1$. 
Proof. Assume $K<H$. Then $|H: K|$ is non-zero but the multiplication by $I_{K}^{H} R_{K}^{H}$ is zero since it factors through $S_{H, V}(K)=0$. Thus $S_{H, V}$ cannot be cohomological. Conversely, $S_{1, V}$ is cohomological because it is $F P_{V}$.

(16.9) Corollary. Assume $k$ has characteristic zero or prime to $|G|$ and let $M$ be a Mackey functor for $G$ over $k$. Then $M$ is cohomological if and only if $M=F P_{V}$ for some $k G$-module $V$.

Proof. By semi-simplicity of Mackey functors and the proposition, a cohomological Mackey functor $M$ is a direct sum of functors of the form $S_{1, V}=F P_{V}$. Alternatively, use Theorem 16.5.

Now we move to characteristic $p$. So from now on $k$ denotes a field whose characteristic is a prime divisor $p$ of $|G|$. In the special case where $G$ is a $p$ group, an analysis of the projective covers of the simple functors in $\operatorname{Comack}_{k}(G)$ can be found in Section 5 of Tambara's paper [18]. Our next result answers this question in general.

(16.10) Proposition. Let $k$ be a field of characteristic $p$ and let $S_{H, V}$ be a simple Mackey functor for $G$ over $k$. Then

(i) $S_{H, V}$ is cohomological if and only if $H$ is a p-group.

(ii) If $H$ is a p-group, the projective cover of $S_{H, V}$ in $\operatorname{Comack}_{k}(G)$ is $F P_{U}$, where $U$ is the trivial source module for $G$ with vertex $H$ and Green correspondent $P_{V}$. (Here $P_{V}$ denotes the projective cover of $V$ as a $k \bar{N}_{G}(H)$-module.)

Proof. If $H$ is not a $p$-group, then there exists a proper subgroup $K$ of index prime to $p$. Then $|H: K|$ is non-zero but the multiplication by $I_{K}^{H} R_{K}^{H}$ is zero since it factors through $S_{H, V}(K)=0$. Thus $S_{H, V}$ is not cohomological. Assume now that $H$ is a $p$-group and let $P_{H, V}$ be the projective cover of $S_{H, V}$ as a Mackey functor. Then we know by 12.4 and 12.7 that there is a surjective morphism $P_{H, V} \rightarrow F P_{U}$ where $U=P_{H, V}(1)$ is the trivial source module of the statement. Therefore there is a surjective morphism $F P_{U} \rightarrow S_{H, V}$. This proves that $S_{H, V}$ is cohomological and that the projective cohomological Mackey functor $F P_{U}$ is its projective cover.

(16.11) Corollary. $\operatorname{Comack}_{k}(G)$ is a subcategory of $\operatorname{Mack}_{k}(G, 1)$.

Proof. The composition factors of a cohomological Mackey functor have the form $S_{H, V}$ with $H$ a $p$-group, and these belong to $\operatorname{Mack}_{k}(G, 1)$.

Dualizing 16.10 we obtain the injective objects in $\operatorname{Comack}_{k}(G)$. Note that the dual of a trivial source module has again trivial source and that the Green correspondence commutes with taking duals. Thus using 4.1 we get:

(16.12) Corollary. If $H$ is a p-group, the injective hull in $\operatorname{Comack}_{k}(G)$ of $S_{H, V}$ is the fixed quotient functor $F Q_{U}$, where $U$ is the trivial source module for $G$ with vertex $H$ and Green correspondent $P_{V}$.

We will need to know in Section 17 that some Mackey functors which play an important rôle in this paper are cohomological. We first need a lemma. 
(16.13) Lemma. Let $P$ be a normal p-subgroup of a finite group $L$. If $M$ is a cohomological Mackey functor for $L / P$, then $\operatorname{Inf}_{L / P}^{L} M$ is a cohomological Mackey functor for $L$.

Proof. Write Inf for $\operatorname{Inf}_{L / P}^{L}$ and let $X \leq Y \leq L$. We have to show that $I_{X}^{Y} R_{X}^{Y}$ is multiplication by $|Y: X|$.

If $P \& Y$, then $(\operatorname{Inf} M)(X)=0=(\operatorname{Inf} M)(Y)$ and there is nothing to prove.

If $P \leq X$, then $(\operatorname{Inf} M)(X)=M(X / P)$ and $(\operatorname{Inf} M)(Y)=M(Y / P)$. The result follows because $M$ is cohomological.

If $P \leq X$ but $P \leq Y$, then $(\operatorname{Inf} M)(X)=0$ and $(\operatorname{Inf} M)(Y)=M(Y / P)$. The result follows because $|Y: X|$ is divisible by $|X P: X|=|P: X \cap P|$ which is a power of $p$.

(16.14) Proposition. Let $k$ be a field of characteristic $p$. If $H$ is a p-group, $\left(\operatorname{Inf}_{\bar{N}_{G}(H)}^{N_{G}(H)} F P_{V}\right) \uparrow_{N_{G}(H)}^{G}$ is a cohomological Mackey functor.

Proof. By 16.6 and 16.13, the property of being cohomological is preserved by both inflation (with a normal $p$-subgroup) and induction.

We conclude by pointing out the relationship between the two categories $\operatorname{Mack}_{R}(G)$ and $\operatorname{Comack}_{R}(G)$ in terms of the Mackey algebra, and what one might call the cohomological Mackey algebra. This latter algebra would be defined as the path algebra modulo an ideal of relations of the same quiver with relations which defined $\mu_{R}(G)$, together with the additional relations $I_{K}^{H} R_{K}^{H}=$ $|H: K| I_{H}^{H}$ for all subgroups $K \leq H \leq G$. It is immediate that cohomological Mackey functors are identified as the modules for this quotient algebra of $\mu_{R}(G)$. However, we should notice by the work of Yoshida [24] (used by Tambara [18]) that the algebra with these generators and relations is none other than the opposite of a Hecke algebra, $\mathscr{E}=\operatorname{End}_{R G}\left(\bigoplus_{H \leq G} R \uparrow_{H}^{G}\right)^{\text {op }}$, and so we have a surjection $\alpha: \mu_{R}(G) \rightarrow \mathscr{E}$, where $\mathscr{E}$ is isomorphic to the cohomological Mackey algebra. Specifically, $\alpha\left(R_{K}^{H}\right): R \uparrow_{K}^{G} \rightarrow R \uparrow_{H}^{G}$ is the natural projection, $\alpha\left(I_{K}^{H}\right): R \uparrow_{H}^{G} \rightarrow R \uparrow_{K}^{G}$ is $x \otimes_{R H} 1 \mapsto \sum_{h \in[H / K]} x h \otimes_{R K} 1$ and $\alpha\left(c_{g}\right): R \uparrow_{H}^{G} \rightarrow R \uparrow_{H g}^{G}$ is $x \otimes 1 \mapsto x g \otimes 1$, the effect of these maps on other components in the direct sum being zero.

Another way to view this homomorphism is that $\mu_{R}(G)$ may be identified as the opposite of the endomorphism ring of its regular representation, which is $\bigoplus_{H \leq G} B^{H} \uparrow_{H}^{G}$. Any such endomorphism yields an endomorphism of the evaluation at 1 of this regular representation, namely $\bigoplus_{H \leq G} B^{H} \uparrow_{H}^{G}(1) \cong \bigoplus_{H \leq G} R \uparrow_{H}^{G}$, and this defines the effect of $\alpha$.

Cohomological Mackey functors are viewed as Mackey functors by restriction along $\alpha$; equally, given a Mackey functor $M$ we may form $\mathscr{E} \otimes_{\mu(G)} M$, which is the largest quotient of $M$ which is cohomological. Taking $M=P_{H, V}$ to be a projective, $\mathscr{E} \otimes_{\mu(G)} P_{H, V}$ is again projective (in $\operatorname{Comack}_{R}(G)$ ), and since the simples in $\operatorname{Comack}_{R}(G)$ are a subset of the simples in $\operatorname{Mack}_{R}(G), \mathscr{E} \otimes_{\mu(G)} P_{H, V}$ still has a unique simple quotient and so is indecomposable (or zero). In fact this quotient is $\mathscr{E} \otimes_{\mu(G)} S_{H, V}$, which equals $S_{H, V}$ if $H$ is a $p$-group, and is 0 otherwise. Thus $\mathscr{E} \otimes_{\mu(G)} P_{H, V}=F P_{U}$ is the projective cover of $S_{H, V}$ in Comack $_{R}(G)$ when $H$ is a $p$-group (here $\left.U=P_{H, V}(1)\right)$ and is zero otherwise. We conclude that the largest quotient of $P_{H, V}$ which is cohomological is $F P_{U}$ if $H$ is a $p$-group, and is zero otherwise. 


\section{BLOCKS OF MACKEY FUNCTORS}

In this section, we describe how Mackey functors for $G$ over a field $k$ of characteristic $p$ are distributed in blocks. The result appears also in a different form (and without proof) in Yoshida's paper [25, 3.4]. Recall that it suffices to describe the block distribution of simples and that this is obtained by considering the transitive closure of the relation linking two simples when there is a non-split extension between them (one way or the other).

We already know that the primitive idempotents of the Burnside algebra $B(G)$ over $k$ split Mackey functors and therefore it suffices to consider the block decomposition of each category $\operatorname{Mack}_{k}(G, J)$ where $J$ is a $p$-perfect subgroup of $G$. Recall that a simple functor $S_{K, W}$ belongs to $\operatorname{Mack}_{k}(G, J)$ if and only if $O^{p}(K)={ }_{G} J$. Now by 10.1 we have an equivalence of categories $\operatorname{Mack}_{k}\left(\bar{N}_{G}(J), 1\right) \longrightarrow \operatorname{Mack}_{k}(G, J)$ so that it suffices to treat the case $J=1$. Thus we have to consider $\operatorname{Mack}_{k}(G, 1)$ and its simple Mackey functors $S_{P, W}$ indexed by $p$-subgroups $P$ of $G$.

We are going to prove that the blocks of $\operatorname{Mack}_{k}(G, 1)$ correspond in a natural fashion to the ordinary blocks of $k G$. Let us first recall a few facts from block theory (see for instance [4] or [6, $§ 58]$ ). By a block of $G$, we mean a primitive idempotent of the centre $Z(k G)$ of $k G$. A $k G$-module $V$ is said to belong to the block $b$ if $b \cdot V=V$. For every $p$-subgroup $P$ of $G$, the Brauer morphism is a ring homomorphism

$$
\mathrm{Br}_{P}: Z(k G) \rightarrow Z\left(k C_{G}(P)\right)^{N_{G}(P)},
$$

where $Z\left(k C_{G}(P)\right)^{N_{G}(P)}$ denotes the subring of $Z\left(k C_{G}(P)\right)$ consisting of $N_{G}(P)$ fixed points. If $b$ is a block of $G$, then $\operatorname{Br}_{P}(b)$ breaks up as an orthogonal sum of primitive idempotents in $Z\left(k C_{G}(P)\right)^{N_{G}(P)}$. Each block $e$ of $N_{G}(P)$ actually lies in $Z\left(k C_{G}(P)\right)^{N_{G}(P)}$, where it is the sum of conjugate primitive idempotents, and so there is a unique block $b$ of $G$ such that $\operatorname{Br}_{P}(b) \cdot e=e$. This unique block will be written $b=e^{G}$.

Let $P$ be a $p$-subgroup of $G$ and $\bar{N}_{G}(P)=N_{G}(P) / P$. Any simple $k \bar{N}_{G}(P)$ module $V$ can be viewed as a $k N_{G}(P)$-module, and as such, it belongs to a block $e$ of $N_{G}(P)$. Thus to every simple Mackey functor $S_{P, V}$, one can associate a block $e$ of $N_{G}(P)$.

(17.1) Theorem. Let $S_{P, V}$ and $S_{Q, W}$ be two simple Mackey functors, where $P$ and $Q$ are p-subgroups of $G$. Let $e$ be the block of $N_{G}(P)$ which $V$ belongs to and $f$ the block of $N_{G}(Q)$ which $W$ belongs to. Then $S_{P, V}$ and $S_{Q, W}$ belong to the same block of Mackey functors if and only if the corresponding blocks $e^{G}$ and $f^{G}$ of $G$ are equal. Thus the blocks of $\operatorname{Mack}_{k}(G, 1)$ are in bijection with the blocks of $k G$, via the map sending the block containing $S_{P, V}$ to the block $e^{G}$ of $k G$.

Proof. Suppose that $S_{P, V}$ and $S_{Q, W}$ belong to the same block of Mackey functors. As mentioned above, by transitivity of the relation linking simples in a block, one can assume that there is a non-split extension

$$
0 \rightarrow S_{P, V} \rightarrow M \rightarrow S_{Q, W} \rightarrow 0
$$


If $P={ }_{G} Q$, then one can assume that $P=Q$ and by 14.3(ii) the sequence of $k N_{G}(P)$-modules

$$
0 \rightarrow V \rightarrow M(P) \rightarrow W \rightarrow 0
$$

is not split. Therefore $V$ and $W$ belong to the same block of $N_{G}(P)$, that is $e=f$. Hence $e^{G}=f^{G}$.

Now we claim that it suffices to treat the case $P<_{G} Q$. Indeed the case $Q<_{G} P$ reduces to the other one by duality, because in the sequence of dual Mackey functors

$$
0 \rightarrow S_{Q, W^{*}} \rightarrow M^{*} \rightarrow S_{P, V^{*}} \rightarrow 0,
$$

the left-hand side is now indexed by the smaller of the two subgroups. Moreover $V^{*}$ belongs to the dual block $\bar{e}$ and the correponding block of $G$ is $\bar{e}^{G}=\overline{e^{G}}$, the dual of $e^{G}$. This last fact can be easily seen as follows: taking duals of blocks consists in applying the canonical involution - of the group algebra (which inverts group elements) and moreover the Brauer morphism commutes with this involution.

Assume now that $P<_{G} Q$. Without loss of generality, we can take $P<$ $Q$. By Theorem 14.3(iii) we know that $M$ is isomorphic to a subfunctor of $\left(\operatorname{Inf}_{\bar{N}_{G}(P)}^{N_{G}(P)} F P_{V}\right) \uparrow_{N_{G}(P)}^{G}$ and therefore by Proposition 16.14, $M$ is a cohomological Mackey functor. Let $F P_{U}$ be the projective cover of $S_{Q, W}$ as a cohomological Mackey functor; thus by Proposition 16.10, $U$ is the trivial source module for $G$ with vertex $Q$ and Green correspondent $P_{W}$, the projective cover of $W$ as $k \bar{N}_{G}(Q)$-module. Since the extension $(*)$ is a non-split extension of cohomological Mackey functors, $M$ is isomorphic to a quotient of $F P_{U}$. Notice incidentally that we have proved that the blocks of $\operatorname{Mack}_{k}(G, 1)$ coincide with the blocks of $\operatorname{Comack}_{k}(G)$.

For any $k G$-module $X$ consider the quotient of the $Q$-fixed points

$$
\overline{X^{Q}}=X^{Q} / \sum_{L<Q} \operatorname{tr}_{L}^{Q}\left(X^{L}\right)
$$

and the canonical map $\mathrm{Br}_{Q}: X^{Q} \rightarrow \overline{X^{Q}}$. If $X$ happens to be a $G$-algebra, then $\mathrm{Br}_{Q}$ is an algebra homomorphism. If $X$ is the $G$-algebra $k G$, then the Brauer morphism defined above is the restriction to $Z(k G)$ of the morphism $\mathrm{Br}_{Q}: k G^{Q} \rightarrow \overline{k G^{Q}}=k C_{G}(Q)$.

Now by [5], $\overline{U^{Q}}=P_{W}$, the projective cover of $W$ as $k \bar{N}_{G}(Q)$-module. Therefore $W$ is a quotient of $\overline{U^{Q}}$. Similarly we want to show that $V$ is a quotient of $\overline{U^{P}}$. Since $P<Q$ and since $S_{Q, W}(P)=0$, we have $M(P)=$ $S_{P, V}(P)=V$. For similar reasons, $M(L)=0$ for every proper subgroup $L$ of $P$. Since $M$ is a quotient of $F P_{U}$, there is a commutative diagram

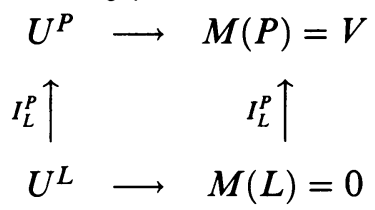

and this proves that $V$ is a quotient of $\overline{U^{P}}$.

Let $b$ be the block of $G$ to which $U$ belongs. Then there is a morphism of $G$-algebras $k G \cdot b \longrightarrow \operatorname{End}_{k}(U)$ mapping $b$ to 1 and this induces a morphism

$$
\overline{(k G \cdot b)^{Q}}=\overline{(k G)^{Q}} \cdot \operatorname{Br}_{Q}(b) \longrightarrow \overline{\operatorname{End}_{k}(U)^{Q}} \cong \operatorname{End}_{k}\left(\overline{U^{Q}}\right) \text {. }
$$


Note the isomorphism on the right is proved in [5, (3.3)]. It follows that $\mathrm{Br}_{Q}(b)$ acts as the identity on $\overline{U^{Q}}$, hence also on its quotient $W$. Since $W$ belongs to the block $f$, this means that $f$ appears in a decomposition of $\mathrm{Br}_{Q}(b)$, that is, $\operatorname{Br}_{Q}(b) \cdot f=f$. In other words $f^{G}=b$. Similarly, $\operatorname{Br}_{P}(b)$ acts as the identity on $\overline{U^{P}}$, hence also on $V$, and it follows that $e^{G}=b$. Thus we have established that $e^{G}=f^{G}$, completing the first part of the proof.

Assume now that $e^{G}=f^{G}$. Consider again the surjection $F P_{U} \rightarrow S_{Q, W}$, that is the projective cover of $S_{Q, W}$ as a cohomological Mackey functor. The argument of the first part of the proof shows that the trivial source module $U$ belongs to the block $f^{G}$. Similarly let $F P_{T} \rightarrow S_{P, V}$ be the projective cover of $S_{P, V}$ as a cohomological Mackey functor. Then $T$ is an indecomposable trivial source module for $G$ which belongs to the block $e^{G}$. Since $S_{Q, W}$ belongs to the same block of Mackey functors as $F P_{U}$, and since $S_{P, V}$ belongs to the same block as $F P_{T}$, it suffices to prove that $F P_{U}$ and $F P_{T}$ lie in the same block. But our assumption tells us that $U$ and $T$ lie in the same block $e^{G}=f^{G}$ of $G$. Therefore we only have to prove that this relation is preserved by passage to fixed point functors. But this fact is easy, because if $X$ and $Y$ are indecomposable $k G$-modules having a common composition factor $Z$, then $F P_{X}$ and $F P_{Y}$ have a common composition factor, namely $S_{1, Z}$. ing.

A practical way of finding in which block lies a Mackey functor is the follow-

(17.2) Corollary. Let $M$ be a Mackey functor such that $M(1) \neq 0$. If $M$ lies in a single block $B$ of $\operatorname{Mack}_{k}(G, 1)$ (e.g., if $M$ is indecomposable), then $M(1)$ lies in a single block $b$ of $k G$, and the block $B$ corresponds to $b$.

Proof. Let $V$ be any composition factor of $M(1)$. By 6.3, $S_{1, V}$ is a composition factor of $M$, hence lies in $B$. By 17.1, $B$ corresponds to the block $b$ of $k G$ containing $V$. Since this holds for every composition factor $V$ of $M(1)$, the whole module $M(1)$ lies in $b$.

We can now improve the result 13.2 on projective simple Mackey functors.

(17.3) Corollary. The following conditions on a simple Mackey functor $S_{H, V}$ are equivalent.

(i) $S_{H, V}$ is a projective simple Mackey functor.

(ii) $S_{H, V}$ is the only simple Mackey functor in its block.

(iii) $H$ is a p-perfect subgroup of $G$ and $V$ is a projective simple $k \bar{N}_{G}(H)$ module.

Proof. (i) $\Rightarrow$ (ii) is clear.

(ii) $\Rightarrow$ (iii) If $J=O^{p}(H)$, the equivalence of categories 10.1 allows us to assume that $J=1$, so that $H$ is a $p$-group. By Theorem $17.1 S_{H, V}$ lies in the same block as some $S_{1, W}$ and therefore $H=1$. Let $b$ be the block of $G$ to which $V$ belongs. If $P$ is a $p$-subgroup such that $\operatorname{Br}_{P}(b) \neq 0$, then there exists a simple $k \bar{N}_{G}(P)$-module $W$ such that $\operatorname{Br}_{P}(b) \cdot W \neq 0$. By the theorem $S_{P, W}$ belongs to the same block as $S_{1, V}$ and therefore $P=1$. This proves that $\operatorname{Br}_{P}(b)=0$ for all non-trivial $p$-subgroups $P$, showing that $b$ is a block of defect zero. Thus its simple module $V$ is projective.

(iii) $\Rightarrow$ (i) This follows immediately from 13.1, as in the proof of 13.2. 


\section{The REPRESENTATION TYPE OF MACKEY FUNCTORS}

We recall that an algebra is said to be of finite representation type if it has only finitely many isomorphism classes of finitely generated indecomposable modules. We also speak of the category of modules as being of finite representation type in this case. If $k$ is a field of characteristic $p$, it is well-known that $k G$-mod has finite representation type if and only if a Sylow $p$-subgroup of $G$ is cyclic [12]. Our main result settles the analogous question for $\operatorname{Mack}_{k}(G)$. In the special case where $G$ has a Sylow $p$-subgroup which is cyclic and normal, the result has been obtained independently by Wiedemann [22], who analyzes explicitly the quiver of the Mackey algebra in this case.

(18.1) Theorem. Let $k$ be a field of characteristic $p$ and let $C$ be a Sylow p-subgroup of $G$. The following conditions are equivalent.

(i) $\operatorname{Mack}_{k}(G)$ has finite representation type.

(ii) $\operatorname{Comack}_{k}(G)$ has finite representation type.

(iii) The Hecke algebra $\operatorname{End}_{k G}\left(\bigoplus_{H \leq G} k \uparrow_{H}^{G}\right)$ has finite representation type.

(iv) $|C|=1$ or $|C|=p$ (or in other words $p^{2} \chi|G|$ ).

Proofs. It is clear that (i) implies (ii), and (ii) $\Leftrightarrow$ (iii) is immediate from Yoshida's theorem [24].

Proof of (ii) $\Rightarrow$ (iv). We assume that $p^{2}$ divides the order of $G$ and we have to prove that $\operatorname{Comack}_{k}(G)$ has infinite representation type. Thus $G$ contains a subgroup $H$ which is either isomorphic to the cyclic group $C_{p^{2}}$ or to the elementary abelian group $C_{p} \times C_{p}$. In the latter case, the category $k G$-mod of (finitely generated) $k G$-modules has infinite representation type [12]. Since there is a full and faithful functor

$$
k G-\bmod \rightarrow \operatorname{Comack}_{k}(G) ; \quad V \mapsto F P_{V},
$$

it is clear that $\operatorname{Comack}_{k}(G)$ has infinite representation type.

Consider now the case $H=C_{p^{2}}$. It suffices to show that $\operatorname{Comack}_{k}(H)$ has infinite representation type. Indeed for each indecomposable $N \in \operatorname{Comack}_{k}(H)$ one can choose an indecomposable summand $M_{N}$ of $N \uparrow_{H}^{G}$ such that $N$ is a direct summand of $M_{N} \downarrow_{H}^{G}$ (because $N$ is a direct summand of $N \uparrow_{H}^{G} \downarrow_{H}^{G}$ ). Only finitely many indecomposable functors $N$ can give rise to isomorphic functors $M_{N}$ because $N$ is a direct summand of $M_{N} \downarrow_{H}^{G}$. This proves that $\operatorname{Comack}_{k}(G)$ has infinite representation type if so has $\operatorname{Comack}_{k}(H)$.

To show that $\operatorname{Comack}_{k}(H)$ has infinite representation type, we simply exhibit infinitely many indecomposable cohomological Mackey functors for $H=C_{p^{2}}$. Let $h$ be a generator of $H$ and let $K=\left\langle h^{p}\right\rangle$ be the subgroup of order $p$. For an arbitrary integer $n$ let $M(1)$ be a $2 n$-dimensional $k$-vector space with basis

$$
x_{0}, y_{0}, x_{1}, y_{1}, \ldots, x_{n-1}, y_{n-1}
$$

and with action of $H$ given by

$$
(h-1) x_{i}=y_{i}, \quad(h-1) y_{i}=0 .
$$

Let $M(K)$ be a $2 n$-dimensional $k$-vector space with basis

$$
u_{0}, v_{0}, u_{1}, v_{1}, \ldots, u_{n-1}, v_{n-1}
$$


and with action of $H / K$ given by

$$
(h-1) u_{i}=v_{i}, \quad(h-1) v_{i}=0 .
$$

Thus both $M(1)$ and $M(K)$ are direct sums of $n$ copies of the 2-dimensional indecomposable $k H$-module. If $p=2$, then $M(K)$ is a free $k[H / K]$-module and this forces us to introduce an $n$-dimensional vector space $M(H)$. Thus we set

$$
M(H)= \begin{cases}0 & \text { if } p \text { is odd } \\ \left\langle w_{0}, \ldots, w_{n-1}\right\rangle & \text { if } p=2 .\end{cases}
$$

Now define restriction and transfer in the following way. We define

$$
R_{1}^{K}: M(K) \rightarrow M(1)
$$

by

$$
R_{1}^{K}\left(v_{i}\right)=0, \quad R_{1}^{K}\left(u_{i}\right)=y_{i},
$$

and $I_{1}^{K}: M(1) \rightarrow M(K)$ by

$$
I_{1}^{K}\left(y_{i}\right)=0, \quad I_{1}^{K}\left(x_{i}\right)= \begin{cases}v_{i+1} & \text { if } 0 \leq i \leq n-2, \\ \sum_{j=0}^{n-1} \lambda_{j} v_{j} & \text { if } i=n-1,\end{cases}
$$

where the elements $\lambda_{j} \in k$ are such that the polynomial

$$
P(X)=X^{n}+\lambda_{n-1} X^{n-1}+\cdots+\lambda_{0}
$$

is a power of an irreducible polynomial over $k$. For the construction of infinitely many indecomposables, it would suffice to choose $\lambda_{j}=0$ for all $j$, so that $P(X)=X^{n}$, but the general case does not require more effort. When $p=2$, we also have to define restriction and transfer from and to $M(H)$ :

$$
\begin{aligned}
R_{K}^{H}: M(H) \rightarrow M(K) ; & R_{K}^{H}\left(w_{i}\right)=v_{i}, \\
I_{K}^{H}: M(K) \rightarrow M(H) ; & I_{K}^{H}\left(u_{i}\right)=w_{i}, I_{K}^{H}\left(v_{i}\right)=0 .
\end{aligned}
$$

The proof that $M$ is a Mackey functor for $H$ is easy and is left to the reader. We only remark that the extra data when $p=2$ are introduced in order to make the Mackey formula hold. Note that any restriction followed by a transfer is zero, so that $M$ is cohomological.

The Mackey functor $M$ for $p$ odd can be pictured in the following selfexplanatory fashion, where $R=R_{1}^{K}, I=I_{1}^{K}$ and $a=h-1$.

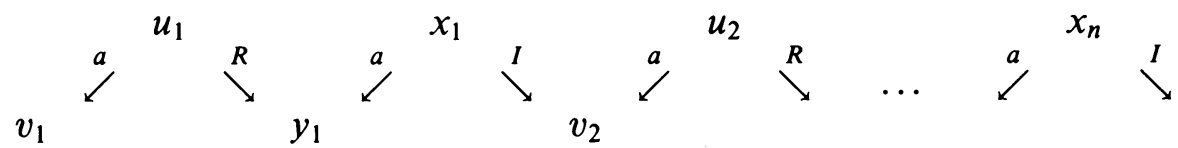

The last arrow $I$ on the right is understood to map $x_{n}$ to the linear combination $\sum_{j=0}^{n-1} \lambda_{j} v_{j}$.

We now recall a standard argument in the representation theory of finite dimensional algebras, showing that $M$ is indecomposable. Let

$$
V=\left\langle v_{0}, \ldots, v_{n-1}\right\rangle=\operatorname{Soc}(M(K))
$$


and define

$$
\phi=I_{1}^{K}(h-1)^{-1} R_{1}^{K}(h-1)^{-1} \in \operatorname{End}_{k}(V) .
$$

Note that it is easy to see that both $I_{1}^{K}(h-1)^{-1}$ and $R_{1}^{K}(h-1)^{-1}$ are welldefined. Thus $V$ becomes a module over the polynomial ring $k[X]$, with $X$ acting via $\phi$.

(18.2) Lemma. The restriction to $V$ defines a surjective ring homomorphism with nilpotent kernel

$$
\pi: \operatorname{End}_{\operatorname{Mack}_{k}(H)}(M) \rightarrow \operatorname{End}_{k[X]}(V) ;\left.\quad f \mapsto f(K)\right|_{V} .
$$

Proof. We assume that $p$ is odd. The necessary modifications when $p=2$ are left to the reader. First it is clear that $f(K)$ must preserve $\operatorname{Soc}(M(K))=V$, because $f(K)$ commutes with the action of $h$. The fact that $\left.f(K)\right|_{V}$ commutes with the endomorphism $\phi$ of $V$ is a straightforward consequence of the fact that $f$ commutes with restriction, transfer and conjugation. Thus $\pi$ is welldefined.

We now prove that $\pi$ is surjective. Let $\left.f(K)\right|_{V}$ be a given endomorphism of $V$ commuting with $\phi$. Extend $f(K)$ to the whole of $M(K)$ by defining $f(K)\left(u_{i}\right)$ to be some element in the inverse image under $(h-1)$ of $f(K)\left(v_{i}\right)$. Then define $f(1)\left(y_{i}\right)=R_{1}^{K} f(K)\left(u_{i}\right)$ and extend $f(1)$ to the whole of $M(1)$ by defining $f(1)\left(x_{i}\right)$ to be some element in the inverse image under $(h-1)$ of $f(1)\left(y_{i}\right)$. By construction, $f$ commutes with $(h-1)$ and with $R_{1}^{K}$. Moreover

$$
\begin{aligned}
I_{1}^{K} f(1)\left(x_{i}\right) & =I_{1}^{K}(h-1)^{-1} f(1)\left(y_{i}\right)=I_{1}^{K}(h-1)^{-1} R_{1}^{K} f(K)\left(u_{i}\right) \\
& =I_{1}^{K}(h-1)^{-1} R_{1}^{K}(h-1)^{-1} f(K)\left(v_{i}\right)=\phi\left(f(K)\left(v_{i}\right)\right) \\
& =f(K)\left(\phi\left(v_{i}\right)\right)=f(K)\left(I_{1}^{K}(h-1)^{-1} R_{1}^{K}(h-1)^{-1}\left(v_{i}\right)\right) \\
& =f(K)\left(I_{1}^{K}\left(x_{i}\right)\right),
\end{aligned}
$$

and this proves that $f$ commutes with $I_{1}^{K}$. Therefore $\pi$ is surjective.

Finally suppose that $f \in \operatorname{Ker}(\pi)$. Then $(h-1) f(K)\left(u_{i}\right)=f(K)\left(v_{i}\right)$ $=0$ so that $f(K)\left(u_{i}\right) \in \operatorname{Ker}(h-1)=V$ and $f(K)^{2}=0$. Now $f(1)\left(y_{i}\right)$ $=R_{1}^{K} f(K)\left(u_{i}\right) \in R_{1}^{K}(V)=0$. Thus $f(1)$ is zero on $\left\langle y_{0}, \ldots, y_{n-1}\right\rangle=$ $\operatorname{Ker}(h-1)=\operatorname{Im}(h-1)$ and again $f(1)^{2}=0$. This completes the proof of the lemma.

Now we can show that $M$ is indecomposable. We have to show that the ring $\operatorname{End}_{\operatorname{Mack}_{k}(H)}(M)$ is local and by the lemma we only have to prove that $\operatorname{End}_{k[X]}(V)$ is a local ring. But since

$$
\phi\left(v_{i}\right)= \begin{cases}v_{i+1} & \text { if } 0 \leq i \leq n-2, \\ \sum_{j=0}^{n-1} \lambda_{j} v_{j} & \text { if } i=n-1,\end{cases}
$$

it is clear that $V$ is generated as a $k[X]$-module by $v_{0}$ and that

$$
V \cong k[X] /(P(X))
$$

where $P(X)=X^{n}+\lambda_{n-1} X^{n-1}+\cdots+\lambda_{0}$. Then $\operatorname{End}_{k[X]}(V) \cong k[X] /(P(X))$ and this is a local ring because $P(X)$ is a power of an irreducible polynomial by assumption.

We have proved that $M$ is an indecomposable cohomological Mackey functor for the cyclic group $H$ of order $p^{2}$. Since $M$ depends on the choice of $n$ 
(and $P(X)$ ), this completes the proof that $\operatorname{Comack}_{k}(H)$ has infinite representation type.

Proof of (iv) $\Rightarrow$ (i). If $|C|=1$, then $p$ does not divide $|G|$ and we know that $\operatorname{Mack}_{k}(G)$ is semi-simple by 3.5 or 14.4 , hence of finite representation type. So we assume now that $C$ has order $p$.

We use the decomposition given by the idempotents in the Burnside ring (9.1 and 9.3(ii))

$$
M=\bigoplus_{\{p \text {-perfect } J\} / G} f_{J} \cdot M
$$

for any Mackey functor $M$. Each summand lies in $\operatorname{Mack}_{k}(G, J)$ and so it suffices to show that $\operatorname{Mack}_{k}(G, J)$ is of finite representation type. By the equivalence of categories 10.1 we reduce to showing that $\operatorname{Mack}_{k}(G, 1)$ has finite representation type. Indeed since $p^{2}$ does not divide $|G|$, a Sylow $p$-subgroup of $\bar{N}_{G}(J)$ has order 1 or $p$ and this applies for each $p$-perfect subgroup $J$.

We use the fact that by 9.7 any $M \in \operatorname{Mack}_{k}(G, 1)$ is projective relative to a Sylow $p$-subgroup $C$ of $G$. Now the argument of D.G. Higman shows that it suffices to prove that $\operatorname{Mack}_{k}(C)$ has finite representation type, since on inducing to $G$ the indecomposable Mackey functors for $C$ we obtain only finitely many indecomposable summands.

To show that $\operatorname{Mack}_{k}(C)$ has finite representation type, we exhibit the quiver with relations corresponding to $\operatorname{Mack}_{k}(C)$ and then we list the finite number of indecomposable representations of this quiver.

Any Mackey functor for the cyclic group $C=\langle h\rangle$ of order $p$ can be viewed as a representation of the following quiver with relations. Let $R=R_{1}^{C}, I=I_{1}^{C}$ and $a=h-1$. Take two vertices 1 and $C$, three arrows

$$
1 \stackrel{I}{\longrightarrow} C, \quad 1 \stackrel{R}{\longleftarrow} C, \quad 1 \stackrel{a}{\longrightarrow} 1,
$$

and three relations

$$
I a=0, \quad a R=0, \quad R I=a^{p-1} .
$$

In the following description of representations, we denote by $V_{k}$ the $k$-dimensional indecomposable representation of the group $C$ over the field $k$. This has a basis $v_{1}, \ldots, v_{k}$, so that $a \cdot v_{i}=v_{i+1}$ for $i<k$ and $a \cdot v_{k}=0$.

There are two simple representations $S_{1,1}$ and $S_{C, 1}$ with

$$
\begin{array}{ll}
S_{1,1}(1)=k, & S_{1,1}(C)=0, \\
S_{C, 1}(1)=0, & S_{C, 1}(C)=k .
\end{array}
$$

Their projective covers (which coincide with their injective hulls) are $P_{1,1}=$ $F P_{k C}$ and $P_{C, 1}=B^{C}$ defined explicitly as follows. First $P_{1,1}(1)=V_{p}$ and $P_{1,1}(C)=k$ with $R(1)=v_{p}$ and $I\left(v_{1}\right)=1$ (the value of $I$ on all other basis elements being zero). On the other hand $P_{C, 1}(1)=k$ and $P_{C, 1}(C)=k^{2}=$ $\langle x, y\rangle$ with $R(x)=0, R(y)=1$ and $I(1)=x$.

Then there are four families of indecomposable representations $A_{k}, B_{k}$, $C_{k}$ and $D_{k}$. In each case the value of the representation at 1 is the module $V_{k}$ and we have to specify the value at $C$ as well as $R$ and $I$. As before we 
only give the definition of $R$ and $I$ on basis elements when it is non-zero.

$$
\begin{aligned}
& A_{k}(C)=k \text {, } \\
& I\left(v_{1}\right)=1 \\
& (1 \leq k \leq p-1) \text {, } \\
& B_{k}(C)=k \text {, } \\
& R(1)=v_{k} \\
& C_{k}(C)=k^{2}=\langle x, y\rangle \text {, } \\
& D_{k}(C)=0 \\
& I\left(v_{1}\right)=x, \quad R(y)=v_{k} \\
& (1 \leq k \leq p-1) \text {, } \\
& (2 \leq k \leq p-1) \text {, } \\
& (2 \leq k \leq p-1) \text {. }
\end{aligned}
$$

This gives the complete list of all indecomposable Mackey functors for $C$ over $k$. The proof of this either follows by direct calculation, or by using the fact that $\mu_{k}(G)$ is a Brauer tree algebra as proved in Section 20, and the classification of indecomposables for such algebras. This completes the proof of the main theorem.

\section{SeLF-INJectivity}

In this section we will assume that our base ring is a field $k$ of characteristic $p$. Using the duality introduced in Section 4, every statement about projective Mackey functors has its counterpart for injective Mackey functors. Thus we do not need to develop a theory of injective Mackey functors over $k$. Our aim in this section is to show that, contrary to the case of group algebras, injectivity is rarely equivalent to projectivity, and we show in Theorem 19.2 that it happens precisely if $p^{2} \chi|G|$.

(19.1) Lemma. Let $k$ be a field of prime characteristic $p$. Every projective $P_{C, V}$ where $|C|=1$ or $p$ is also injective, and $\operatorname{Soc}\left(P_{C, V}\right)=S_{C, V}$.

Proof. In case $C=1$ a proof of this result has already been given in 13.3. The proof we now give works in general, and we suppose $|C|=1$ or $p$. Every projective $P_{C, V}$ is a summand of $B^{C} \uparrow_{C}^{G}$ by Section 8 and since injectivity is preserved by induction and taking direct summands, in order to prove that $P_{C, V}$ is injective it suffices to prove that the projective indecomposable Mackey functor $B^{C}$ is also injective. This is clear if $C=1$ because Mackey functors for the trivial group are just vector spaces. If $C$ is cyclic of order $p$, then we have seen in the proof of 18.1 that $B^{C}$ (written $P_{C, 1}$ in the last section) is both the projective cover and the injective hull of $S_{C, k}^{C}$. In fact it is easy to see that this 3-dimensional Mackey functor is self-dual.

Now to show that $\operatorname{Soc}\left(P_{C, V}\right)=S_{C, V}$ it suffices to show that $\left(P_{C, V}\right)^{*}=$ $P_{C, V^{*}}$ since this has simple top $S_{C, V^{*}}$. By the injectivity of $P_{C, V}$ we know that $\left(P_{C, V}\right)^{*}$ is an indecomposable projective, and it has the form $\left(P_{C, V}\right)^{*}=P_{C, W}$ since its vertex is $C$. Now $W$ is identified as the simple quotient of the Green correspondent $f\left(\left(P_{C, V}\right)^{*}(1)\right)$ by 12.7 . We have

$$
f\left(\left(P_{C, V}\right)^{*}(1)\right)=f\left(\left(P_{C, V}(1)\right)^{*}\right)=\left(f\left(P_{C, V}(1)\right)\right)^{*}
$$

and the unique simple quotient is $\left(\operatorname{Soc}\left(f\left(P_{C, V}(1)\right)\right)\right)^{*} \cong V^{*}$ since $f\left(P_{C, V}(1)\right)=$ $P_{V}$ as $k \bar{N}_{G}(C)$-modules and the top and socle are isomorphic.

Recall that an abelian category is called self-injective if projective and injective objects coincide.

(19.2) Theorem. Let $k$ be a field of prime characteristic $p$. Then $\operatorname{Mack}_{k}(G)$ is self-injective if and only if a Sylow p-subgroup of $G$ is cyclic of order 1 or $p$.

Combining this with the main result of Section 18, we deduce the following corollary. 
(19.3) Corollary. $\operatorname{Mack}_{k}(G)$ is self-injective if and only if it is of finite representation type.

We first need another lemma.

(19.4) Lemma. Let $H$ be a p-group and let $R_{K}^{H}: B(H) \rightarrow B(K)$ be the restriction map to a subgroup $K \leq H$, where $B(H)$ is the Burnside algebra over $k$. If $S$ is a normal subgroup of $H$, then

$$
R_{K}^{H}(H / S)= \begin{cases}K / K \cap S & \text { if } K S=H, \\ 0 & \text { otherwise. }\end{cases}
$$

Proof. This is an easy application of the Mackey formula.

$$
R_{K}^{H}(H / S)=\sum_{h \in[K \backslash H / S]} K / K \cap{ }^{h} S=\sum_{h \in[K S \backslash H]} K / K \cap S=|H: K S| \cdot K / K \cap S
$$

and the result follows.

Proof of Theorem 19.2. Assume first that a Sylow $p$-subgroup of $G$ is of order 1 or $p$. We use the decomposition given by the idempotents in the Burnside ring (Section 9). So it suffices to show that for each $p$-perfect subgroup $J$, the category $\operatorname{Mack}_{k}(G, J)$ is self-injective. By the equivalence of categories 10.1 we reduce to showing that $\operatorname{Mack}_{k}(G, 1)$ is self-injective. Indeed a Sylow $p$-subgroup of $N_{G}(J) / J$ is also of order 1 or $p$. Now the projectives in $\operatorname{Mack}_{k}(G, 1)$ are precisely the $P_{C, V}$ where $|C|=1$ or $p$, and these are also injective by 19.1 .

Assume now that a Sylow $p$-subgroup $H$ of $G$ has order a multiple of $p^{2}$. We have to exhibit a projective Mackey functor which is not injective. Any projective indecomposable $P_{H, V}$ has vertex $H$ and source $B^{H}$, by 11.1. Since $B^{H}$ is a summand of $P_{H, V} \downarrow_{H}^{G}$ and since injectivity is preserved by restriction, it suffices to prove that $B^{H}$ is not injective. Since it is indecomposable and the socle of an injective indecomposable is simple, it is enough to show that the socle of $B^{H}$ is not simple. In fact we prove that $S_{H, k}^{H}$ (which is 1-dimensional on evaluation at $H$ and vanishes at proper subgroups) appears at least twice in the socle of $B^{H}$. The proof divides into two cases.

For the first case, assume that $H$ is not elementary abelian and let $\Phi(H)$ be the Frattini subgroup of $H$, which is not trivial by assumption. Let $J$ be any normal subgroup of $H$ contained in $\Phi(H)$ (and there are at least two of them: $J=1$ or $J=\Phi(H))$. There is a morphism of Mackey functors $f: S_{H, k}^{H} \rightarrow B^{H}$ defined on evaluation at $H$ to be the map $f(H)$ sending the generator 1 of $S_{H, k}^{H}(H)=k$ to the $H$-set $H / J$, viewed as an element of the Burnside algebra $B(H)$. In order to check that $f$ is a morphism of Mackey functors, it suffices to see that $f$ commutes with restriction from $H$ to a proper subgroup $K$ of $H$, because $S_{H, k}^{H}$ vanishes on proper subgroups of $H$. Thus we only have to check that $R_{K}^{H}(H / J)=0$. But this is clear by 19.4 because $K J$ is a proper subgroup of $H$ (since $J \leq \Phi(H)$ ). Thus we have constructed at least two linearly independent morphisms $f: S_{H, k}^{H} \rightarrow B^{H}$ and this proves that the socle of $B^{H}$ is not simple.

For the second case, we assume that $H$ is elementary abelian (of rank $\geq 2$ ). Again we have a morphism $f: S_{H, k}^{H} \rightarrow B^{H}$ defined on evaluation at $H$ to be the 
map $f(H)$ sending the generator 1 of $S_{H, k}^{H}(H)=k$ to the $H$-set $H / 1$. Now we construct another morphism $g: S_{H, k}^{H} \rightarrow B^{H}$ which is linearly independent of $f$ and this will prove that the socle of $B^{H}$ is not simple. Let $\mathscr{C}$ be the set of all subgroups of $H$ of order $p$ (i.e., the lines in the vector space $H$ ). We define $g(H): k \rightarrow B(H)$ to be the map sending 1 to the element $X=\sum_{C \in \mathscr{C}} H / C$. As above it suffices to prove that $R_{K}^{H}(X)=0$ for every proper subgroup $K$ of $H$. By 19.4, we have

$$
R_{K}^{H}(X)=\left\{\begin{array}{cl}
\sum_{C \in \mathscr{C}, K C=H} K / 1 & \text { if } K \text { has index } p, \\
0 & \text { otherwise. }
\end{array}\right.
$$

In the first case, we let $\mathscr{D}$ be the set of all subgroups of $K$ of order $p$ and we have

$$
R_{K}^{H}(X)=(|\mathscr{C}|-|\mathscr{D}|) \cdot K / 1 .
$$

Now if $|H|=p^{n}$, then $|\mathscr{C}|=\left(p^{n}-1\right) /(p-1)$ and $|\mathscr{D}|=\left(p^{n-1}-1\right) /(p-1)$, so that $|\mathscr{C}|-|\mathscr{D}|=p^{n-1}$ is a power of $p$. This shows that $X$ restricts to zero and completes the proof.

We may consider the injective objects in the category $\operatorname{Comack}_{k}(G)$ of cohomological Mackey functors. We know by 16.12 that the injective indecomposable cohomological Mackey functors are the functors $F Q_{V}$ where $V$ is as above. If the characteristic $p$ of $k$ does not divide $|G|$, then $\operatorname{Comack}_{k}(G)$ is semi-simple and $F P_{V}=F Q_{V}$ for all $V$. Otherwise, in contrast with 19.2 above, $\operatorname{Comack}_{k}(G)$ is never self-injective. Indeed it suffices to take $V=k$, the trivial module: all restriction maps in $F P_{k}$ are isomorphisms and some of the induction maps are zero (as soon as $p$ divides the index), while the opposite holds for $F Q_{k}$. Thus $F P_{k}$ cannot be injective and $F Q_{k}$ cannot be projective.

\section{BRAUER TREeS}

In this section we consider a complete discrete valuation ring $\mathscr{O}$ with quotient field $K$ of characteristic 0 and residue field $k$ of characteristic $p$. Recall from the previous two sections that $\operatorname{Mack}_{k}(G)$ has finite-representation type if and only if it is self-injective, and that it happens precisely if $p^{2} \chi|G|$. We go on to show that in this case each block of the Mackey algebra over $k$ is either a Brauer tree algebra or a matrix algebra, and that the Brauer tree may be calculated by decomposition in exactly the same way as for group representations. For the properties of Brauer tree algebras we refer to [3]. Since the Brauer trees give total information about the structure of projective modules [3] as well as decomposition, and since also there is a well-known classification of indecomposable representations (and also the Auslander-Reiten quiver) in this situation, we use these trees to specify all this information when we give tables in Section 21.

We first start with the situation where $G$ has a normal Sylow $p$-subgroup $C$ of order $p$. We deal only with the projectives of the form $P_{1, V}$ and $P_{C, V}$ since the other projectives may be reduced to this case using the equivalence of categories 10.1. Now these projectives are indexed by letting $V$ range through the complete set of simple $k G$-modules, since the normal $p$-subgroup $C$ must act trivially on such $V$ and so we may regard $V$ either as a $k G$-module or as a $k[G / C]$-module. 
We obtain the structure of $P_{1, V}$ by means of the isomorphism $P_{1, V} \cong F P_{P_{V}}$ given in 13.3, where $P_{V}$ is the projective cover of $V$ as a $k G$-module. These modules $P_{V}$ are uniserial, being given by a Brauer tree which is a star [3], and within any block we may number the simples $V_{1}, \ldots, V_{e}$ so that

$$
P_{V_{i}}=\begin{aligned}
& V_{i} \\
& V_{i+1} \\
& \vdots \\
& V_{i-1} \\
& V_{i}
\end{aligned}
$$

with a cyclic ordering of the $i$ 's. We have $F P_{P_{V_{i}}}(C)=V_{i}$ by $[3, \mathrm{p} .37]$. Thus the composition factors of $F P_{P_{V_{i}}}$ are $S_{1, V_{i}}, S_{1, V_{i+1}}, \ldots, S_{1, V_{i-1}}, S_{1, V_{i}}$ and $S_{C, V_{i}}$ by 6.3 , and by the method of Section 6 using the map $\psi$. Here we use the fact that each of the $S_{1, V_{j}}$ vanishes at $C$. By 14.3 the only possible non-zero Ext groups between these simples are between $S_{1, V_{i}}$ and $S_{C, V_{i}}$, and between $S_{C, V_{i}}$ and $S_{1, V_{i}}$, where the Ext groups have dimension 1, and also between $S_{1, V_{j}}$ and $S_{1, V_{j+1}}$ for $j=1, \ldots, e$, where the Ext group has dimension $\leq 1$.

We conclude that the top two Loewy layers of $P_{1}, V_{i}$ must be either

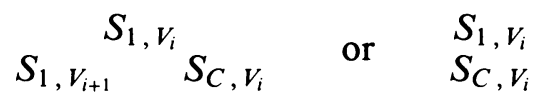

and we eliminate the second of these using the fact that $P_{1, V_{i}}$ is also the injective hull of $S_{1, V_{i}}$ by 13.3 , and there must also be a subfunctor

$$
\begin{aligned}
& S_{C, V_{i}} \\
& S_{1, V_{i}} .
\end{aligned}
$$

Now using the remaining restriction on Ext groups we obtain that $P_{1, V_{i}}$ has a diagram

$$
\begin{gathered}
S_{1, V_{i}} \\
S_{1, V_{i+1}} \\
\vdots \\
S_{1, V_{i-1}} S_{C, V_{i}} \\
S_{1, V_{i}} .
\end{gathered}
$$

We turn now to the indecomposable projectives $P_{C}, V_{i}$ for which we use decomposition theory. We shall use superscripts $K$ or $k$ to indicate the field over which the simple Mackey functors are defined. Observe that $V_{i}$ lifts to a simple $K G$-module also written $V_{i}$ because $p$ does not divide $|G / C|$. By 9.10 , the decomposition of $S_{1, V_{i}}^{K}$ and $S_{C, V_{i}}^{K}$ is completely determined by the decomposition of their evaluations at $p$-subgroups, namely 1 and $C$. Since $S_{C, V_{i}}^{K}$ vanishes at 1 , we obtain $d\left(S_{C, V_{i}}^{K}\right)=S_{C, V_{i}}^{k}$. Now $S_{1, V_{i}}^{K}=F P_{V_{i}}$ has value $V_{i}$ at both 1 and $C$, and since $S_{1, V_{i}}^{k}$ vanishes at $C$, we obtain $d\left(S_{1, V_{i}}^{K}\right)=S_{1, V_{i}}^{k}+S_{C, V_{i}}^{k}$. This works for every simple $K[G / C]$-module $V_{i}$. Moreover the other characteristic zero simple Mackey functors do not decompose with $S_{C, V_{i}}^{k}$ as a composition factor, because they all vanish at $C$, since $C$ acts non-trivially on them. Using the fact that the Cartan matrix is the product of the decomposition matrix and its transpose, we deduce that the only composition factors of $P_{C, V_{i}}^{k}$ are $S_{C, V_{i}}^{k}$ 
with multiplicity 2 and $S_{1, V_{i}}^{k}$ with multiplicity 1 . Returning to the situation where $k$ is our sole field of definition (and thus omitting superscripts), we have $\operatorname{Ext}\left(S_{C, V_{i}}, S_{C, V_{i}}\right)=0$ by 14.3 . The only possibility is that $P_{C, V_{i}}$ has a diagram

$$
\begin{gathered}
S_{C, V_{i}} \\
S_{1, V_{i}} \\
S_{C, V_{i}} .
\end{gathered}
$$

We may now sum up the results of our calculations in the following way.

(20.1) Theorem. Let $k$ be a field of characteristic $p$ and let $G$ be a group with $a$ cyclic normal Sylow p-subgroup of order $p$. Let $b$ be a block of Mackey functors lying in $\operatorname{Mack}_{k}(G, 1)$. Then $b$ is a Brauer tree algebra with Brauer tree having the form of a star with arms of length 2 .

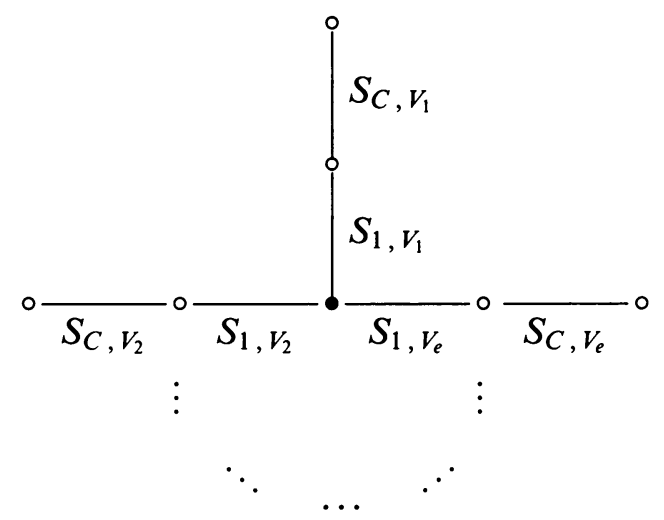

The inner star is the Brauer tree of the corresponding block of $k G$-modules (with exceptional vertex in the center).

Proof. We merely have to observe that the indecomposable projective Mackey functors have the structure determined by this tree, as described in [3].

Now we can state the general result.

(20.2) Theorem. Let $k$ be a field of characteristic $p$ and let $G$ be a group for which $p^{2} \backslash|G|$. Then each block of $\mu_{k}(G)$ is either a matrix algebra over $a$ division ring, or a Brauer tree algebra.

Proof. Since the types of algebra mentioned are stable under Morita equivalence, by the equivalence of categories $\operatorname{Mack}_{k}(\bar{N}(J), 1) \approx \operatorname{Mack}_{k}(G, J)$ it suffices to prove the result for blocks in $\operatorname{Mack}_{k}(G, 1)$. We suppose we have a block $\hat{b}$ of Mackey functors of this form, which is associated by 17.1 to a block $b$ of representations of $G$. By $17.3 \hat{b}$ is a matrix algebra if and only if $b$ is a block of defect zero. Thus we now suppose that $b$ has defect $C$ where $C$ is a Sylow $p$-subgroup of order $p$.

Let $e$ be the unique block of $N_{G}(C)$ for which $b=e^{G}$ and let $\hat{e}$ be the corresponding block of $\operatorname{Mack}_{k}\left(N_{G}(C), 1\right)$. We claim that the Green correspondence provides a stable equivalence between $\hat{b}$ and $\hat{e}$. The arguments we use here are similar to the case of group representations, with some modifications. We must show that Green correspondence provides a bijection between the (isomorphism classes of) indecomposable non-projective Mackey functors 
in $\hat{b}$ and in $\hat{e}$, and also that the corresponding groups of homomorphisms modulo projectives are isomorphic. We start by observing that by 9.7 all the indecomposables in these blocks which are not 1-projective have $C$ as a vertex, so the Green correspondence applies. Also the Green correspondent of a projective (with vertex $C$ ) is again a projective since the restriction and induction of projectives are projective. Thus the non-projective Mackey functors correspond.

It is now necessary to prove that an indecomposable non-projective Mackey functor $M$ is in $\hat{b}$ if and only if its Green correspondent $f(M)$ is in $\hat{e}$. To do this, notice that if $V$ is a simple $k G$-module in $b$ so that $S_{1, V}^{G} \in \hat{b}$, then $f\left(S_{1, V}^{G}\right)=S_{1, f(V)}^{N(C)}$ by 11.6 , and this lies in $\hat{e}$ by 17.2 since its evaluation at 1 , namely $f(V)$, lies in $e$. Thus there is at least one Mackey functor in $\hat{b}$ whose Green correspondent lies in $\hat{e}$. Now observe that if $L$ and $M$ lie in $\hat{b}$ with vertex $C$ then by the usual argument [3, Section 10]

$$
\operatorname{Hom}_{\text {Mack }_{k}(G)}^{1}(L, M) \cong \operatorname{Hom}_{\operatorname{Mack}_{k}\left(N_{G}(C)\right)}^{1}(f(L), f(M))
$$

where these groups denote homomorphisms modulo 1-projectives. We use again the fact that Green correspondence sends projectives to projectives to deduce that in fact we have an isomorphism

$$
\underline{\operatorname{Hom}}_{\operatorname{Mack}_{k}(G)}(L, M) \cong \underline{\operatorname{Hom}}_{\operatorname{Mack}_{k}\left(N_{G}(C)\right)}(f(L), f(M))
$$

where these groups denote homomorphisms modulo projectives. We deduce that

$$
\operatorname{Ext}_{\mu_{k}(G)}(L, M) \cong \operatorname{Ext}_{\mu_{k}\left(N_{G}(C)\right)}(f(L), f(M))
$$

as in [14, II 5.9]. Since the closure of any non-projective object under the equivalence relation generated by the property of having a non-zero Ext group gives all non-projectives in the block, we deduce for non-projective Mackey functors that $M$ is in $\hat{b}$ if and only if $f(M)$ is in $\hat{e}$. At the same time we have also proved the desired isomorphism of homomoprhisms modulo projectives.

To sum up these arguments, we have shown that Green correspondence gives a stable equivalence of $\hat{b}$ and $\hat{e}$. By $20.1 \hat{e}$ is a Brauer tree algebra. We finally quote the theorem of Gabriel and Riedtmann [10] which states that an algebra stably equivalent to a Brauer tree algebra is a Brauer tree algebra, and this completes the proof.

For the rest of this section, we fix the following notation. We continue to suppose that $G$ is a group whose order is divisible by $p$ but not $p^{2}$. Let $b$ be a block of $G$-modules which is not a matrix algebra, and let $\hat{b}$ be the corresponding block of Mackey functors for $G$. Let $T_{\text {Mod }}$ be the Brauer tree of $b$ and let $T_{\text {Mack }}$ be the Brauer tree of $\hat{b}$.

We want to show that $T_{\text {Mod }}$ is naturally a subgraph of $T_{\text {Mack }}$. We first need an elementary property of graphs. Given a graph with edge set $E$, we define the neighbours of an edge $e$ to be the set of edges

$$
N(e)=\{f \in E \mid f \text { has a vertex in common with } e\} .
$$

Moreover an edge is called a twig if one of its vertices is the vertex of no other edge. 
(20.3) Lemma. Let $T$ be a finite graph which is a disjoint union of trees. Then, apart from isolated vertices, $T$ is determined by knowing for every edge $e$ the set of neighbours $N(e)$.

Proof. There may be edges $e$ for which $N(e)=\{e\}$, namely components consisting just of $e$ and its two end vertices. By removing these we may assume that $N(e) \neq\{e\}$ always. Now for each edge $e$ we partition $N(e)-\{e\}$ into (at most) 2 subsets by means of the equivalence relation $e_{1} \sim e_{2} \Leftrightarrow e_{1} \in N\left(e_{2}\right)$. The edges for which $N(e)-\{e\}$ consists of just one equivalence class are precisely the twigs. By removing such a twig from the situation, together with all occurrences of that twig in the neighbour sets, we reduce to a union of trees of smaller size. Now by induction we can reconstruct the smaller graph, and the twig $e$ we removed is joined on to it at the common vertex of all the edges $N(e)$.

(20.4) Proposition. The edges in $T_{\text {Mack }}$ labelled by simple Mackey functors of the form $S_{1, V}$ span a subtree isomorphic to $T_{\mathrm{Mod}}$. The isomorphism is given by associating to an edge labelled $S_{1, V}$ the edge of $T_{\text {Mod }}$ labelled $V$.

Proof. Let $T$ be the subgraph of $T_{\text {Mack }}$ whose edges are the $S_{1, V}$. Thus $T$ is a union of trees and has no isolated vertices. To show that $T$ and $T_{\text {Mod }}$ are isomorphic it suffices by 20.3 to show that the neighbour sets of edges $V_{0}$ and $S_{1, V_{0}}$ correspond under the correspondence $V \leftrightarrow S_{1, V}$. According to the structure of a Brauer tree algebra, the neighbour sets of each edge of the Brauer tree are precisely the simples which occur as composition factors of the projective cover of the simple labelling that edge. Thus we need to show that $W$ is a composition factor of $P_{V}$ if and only if $S_{1, W}$ is a composition factor of $P_{1, V}$. But by $13.3 P_{1, V}=F P_{P_{V}}$ and the result is clear by 6.3 .

We move now to decomposition theory and for this we suppose that the fields $K$ and $k$ are both splitting fields for the respective Mackey algebras. From the known decomposition theory of blocks with cyclic defect group, we can label the vertices of $T_{\text {Mod }}$ by the simple modules in characteristic zero in such a way that each simple decomposes according to the edges incident with that vertex (each non-zero decomposition number being equal to 1). In fact there is an exceptional vertex which is possibly labelled by several simple modules, but their decompositions are all equal.

We analyse further the structure of the Brauer tree for Mackey functors in $\hat{b}$, and show that its vertices may also be labelled by simple Mackey functors in characteristic zero in such a way that each simple decomposes according to the edges incident with that vertex. Thus the Brauer tree may be calculated from the decomposition map (and conversely) in the same way as for group representations. In the process we shall also see how $T_{\text {Mack }}$ is constructed from $T_{\text {Mod }}$. First note that we can already label the vertices of $T_{\text {Mack }}$ which belong to $T_{\text {Mod }}$, using the already existing labelling of $T_{\text {Mod }}$. Thus we have to find a labelling of the remaining vertices, using the simple Mackey functors $S_{C, W}^{K}$ in characteristic zero which are indexed by $C$ (where $C$ still denotes a Sylow $p$-subgroup of order $p$ ).

As earlier in this section, we use superscripts $K$ or $k$ to indicate the ground field over which Mackey functors are defined. To simplify notation, we shall 
use the letter $U$ to denote simple $K G$-modules and the letter $V$ for simple $k G$-modules. Moreover the simple modules for $N_{G}(C) / C$ will be written with the letter $W$. Since $N_{G}(C) / C$ has order prime to $p$, we can use the same letter $W$ both over $K$ and $k$ (that is, we identify $W$ and its decomposition $d(W))$. The letter $d$ stands for the decomposition of both modules and Mackey functors. We are going to use repeatedly the following consequence of the fact that the Cartan matrix is the product of the decomposition matrix and its transpose. Given two simple Mackey functors $S_{H, V}^{k}$ and $S_{H^{\prime}, V^{\prime}}^{k}$, the Cartan integer $c\left(S_{H, V}^{k}, S_{H^{\prime}, V^{\prime}}^{k}\right)$ is non-zero (that is, the two edges $S_{H, V}^{k}$ and $S_{H^{\prime}, V^{\prime}}^{k}$ of the Brauer tree are neighbours) if and only if there exists a simple Mackey functor $S_{J, U}^{K}$ over $K$ whose decomposition contains both $S_{H, V}^{k}$ and $S_{H^{\prime}, V^{\prime}}^{k}$.

We proceed in a series of lemmas, starting with a general description of $d$.

(20.5) Lemma.

(i) If $U$ is a simple $K G$-module and $d(U)=\sum_{i} V_{i}$, then

$$
d\left(S_{1, U}^{K}\right)=\left(\sum_{i} S_{1, V_{i}}^{k}\right)+X_{U}
$$

where $X_{U}$ is a linear combination of simple Mackey functors $S_{C, W}^{k}$ indexed by $C$.

(ii) $d\left(S_{C, W}^{K}\right)=S_{C, W}^{k}$.

Proof. (i) This is an immediate consequence of 9.10 (with $J=1$ ), using also 6.3. Note that the map $\bar{\psi}$ appearing in 9.10 involves only the two subgroups 1 and $C$.

(ii) This is again an immediate consequence of 9.10 using the fact that $S_{C, W}^{K}$ and $S_{C, W}^{k}$ vanish at 1 .

The first statement above implies that if $U$ labels a vertex of $T_{\text {Mod }}$, so that it decomposes into the edges $V_{i}$ emanating from that vertex, then the simple Mackey functors of the form $S_{1, V}^{k}$ appearing in $d\left(S_{1, U}^{K}\right)$ are precisely the edges emanating from the vertex $S_{1, U}^{K}$ which lie in $T_{\text {Mod }}$ :

We shall also need a technical lemma on exceptional characters, which we only prove under special assumptions (see 20.11 for a more general result).

(20.6) Lemma. Let $U^{1}, \ldots, U^{m}$ be the simple $K G$-modules labelling the exceptional vertex of $T_{\text {Mod }}$.

(i) The dimension of the fixed points $\left(U^{j}\right)^{C}$ is independent of $j$.

(ii) If only one simple Mackey functor $S_{C, W}^{k}$ indexed by $C$ appears in the decomposition of each $S_{1, U^{j}}^{K}$, then for $1 \leq j \leq m$ all of the decomposition numbers $d\left(S_{1, U^{j}}^{K}, S_{C, W}^{k}\right)$ are equal. Thus in the notation of 20.5, $X_{U^{j}}$ is independent of $j$.

Proof. (i) Let $\chi$ be the character of some $U^{j}$. We compute the dimension of fixed points by restricting $\chi$ to $C$ and taking the scalar product with the trivial character. If $u$ denotes a generator of $C$, we have

$$
p\langle\chi, 1\rangle_{C}=\sum_{i=0}^{p-1} \chi\left(u^{i}\right)=\chi(1)+\sum_{i=1}^{p-1} \sum_{\phi} d_{\chi, \phi}^{u^{i}} \phi(1)
$$


where $d_{\chi, \phi}^{u^{i}}$ denotes the higher decomposition number and $\phi$ runs over the set of all Brauer characters of $C_{G}\left(u^{i}\right)=C_{G}(C)$ belonging to the blocks corresponding to the block $b$ of $G$. (In fact by Brauer's second main theorem, these blocks are conjugate in $N_{G}(C)$ and their sum is the block of $N_{G}(C)$ corresponding to $b$. Moreover each of them actually contains a single Brauer character.) Now it is known from the theory of blocks with cyclic defect group that the higher decomposition number $d_{\chi, \phi}^{u}$ corresponding to an exceptional character is a sum of primitive $p$-th roots of unity (which depend on the exceptional character), up to a sign $\delta$ which is independent of the exceptional character:

$$
d_{\chi, \phi}^{u}=\delta\left(\zeta_{1}+\cdots+\zeta_{e}\right)
$$

(Here $e$ is in fact the inertial index.) Moreover the decomposition number corresponding to a power $u^{i}$ of $u$ is simply

$$
d_{\chi, \phi}^{u^{i}}=\delta\left(\zeta_{1}^{i}+\cdots+\zeta_{e}^{i}\right)
$$

Therefore we obtain

$$
p\langle\chi, 1\rangle_{C}=\chi(1)+\sum_{\phi} \phi(1) \delta \sum_{i=1}^{p-1}\left(\zeta_{1}^{i}+\cdots+\zeta_{e}^{i}\right)=\chi(1)-\sum_{\phi} \phi(1) \delta e,
$$

because $\sum_{i=1}^{p-1} \zeta^{i}=-1$ for any primitive $p$ th root of unity $\zeta$. Now the result is independent of the exceptional character, because $\chi(1)$ is the same for all exceptional characters since they all decompose in the same way.

(ii) Let $a_{j}=d\left(S_{1, U^{j}}^{K}, S_{C, W}^{k}\right)$ so that $d\left(S_{1, U^{j}}^{K}\right)=\left(\sum_{i} S_{1, V_{i}}^{k}\right)+a_{j} S_{C, W}^{k}$. As an immediate consequence of 9.10 , we have

$$
\operatorname{dim}\left(\left(U^{j}\right)^{C}\right)=\operatorname{dim}\left(S_{1, U^{j}}^{K}(C)\right)=\left(\sum_{i} \operatorname{dim}\left(S_{1, V_{i}}^{k}(C)\right)\right)+a_{j} \operatorname{dim}\left(S_{C, W}^{k}(C)\right) .
$$

Since $\operatorname{dim}\left(\left(U^{j}\right)^{C}\right)$ is independent of $j$ by part (i), we see that $a_{j}$ is independent of $j$.

Now we establish the link between the tree and the simple Mackey functors $S_{C, W}^{k}$ appearing in the (yet unknown) factor $X_{U}$ of Lemma 20.5.

\section{(20.7) Lemma.}

(i) If $S_{C, W}^{k}$ appears in the decomposition of $S_{1, U}^{K}$, then $S_{C, W}^{k}$ is a neighbour of $S_{1, V_{i}}^{k}$ for all edges $V_{i}$ emanating from $U$.

(ii) If conversely $S_{C, W}^{k}$ is a neighbour of $S_{1, V}^{k}$, then for one end $U$ of the edge $V$ of $T_{\text {Mod }}, S_{C, W}^{k}$ appears in the decomposition of $S_{1, U}^{K}$. Moreover $S_{C, W}^{k}$ is a neighbour of $S_{1, V_{i}}^{k}$ for all edges $V_{i}$ emanating from $U$.

Proof. (i) Let $V_{i}$ be any edge emanating from $U$. Then the decomposition number $d\left(S_{1, U}^{K}, S_{1, V_{i}}^{k}\right)$ is non-zero. By assumption $d\left(S_{1, U}^{K}, S_{C, W}^{k}\right) \neq 0$ and so the Cartan integer $c\left(S_{C, W}^{k}, S_{1, V_{i}}^{k}\right)$ is non-zero.

(ii) By assumption the Cartan integer $c\left(S_{C, W}^{k}, S_{1, V}^{k}\right)$ is non-zero. Therefore there exists a simple Mackey functor $S_{H, U}^{K}$ whose decomposition contains both $S_{C, W}^{k}$ and $S_{1, V}^{k}$. Then $H$ cannot be $C$ by 20.5 (ii), so $H=1$. Thus $V$ 
appears in a decomposition of $U$, and this means that $U$ is one of the ends of the edge $V$ of $T_{\text {Mod }}$. Moreover since $S_{C, W}^{k}$ appears in the decomposition of $S_{1, U}^{K}$, part (i) applies to deal with all edges $V_{i}$ emanating from $U$.

We now refine our knowledge of the Mackey functor tree $T_{\text {Mack }}$ and consider the remaining edges $S_{C, W}^{k}$.

(20.8) Lemma. Each edge $S_{C, W}^{k}$ of $T_{\text {Mack }}$ is a twig, the non-free end of which belongs to the subtree $T_{\text {Mod }}$.

Proof. If $S_{1, V}^{k}$ is a neighbour of $S_{C, W}^{k}$ for some $V$, then $S_{C, W}^{k}$ is fastened to $T_{\text {Mod }}$. Assume now that $S_{C, W^{\prime}}^{k}$ is a neighbour of $S_{C, W}^{k}$ for some $W^{\prime}$. Then there exists a simple Mackey functor $S_{H, U}^{K}$ whose decomposition contains both $S_{C, W}^{k}$ and $S_{C, W^{\prime}}^{k}$. By 20.5 (ii), we must have $H=1$ and by 20.7 (i), $S_{C, W}^{k}$ is a neighbour of $S_{1, V_{i}}^{k}$ for all edges $V_{i}$ emanating from $U$. Thus in all cases $S_{C, W}^{k}$ is fastened to $T_{\text {Mod }}$. Since $T_{\text {Mack }}$ is a tree and $T_{\text {Mod }}$ is connected, the end vertex of $S_{C, W}^{k}$ which does not lie in $T_{\text {Mod }}$ cannot be connected to any other vertex, hence is free. This proves that $S_{C, W}^{k}$ is a twig.

(20.9) Lemma. The free end of the twig $S_{C, W}^{k}$ is not an exceptional vertex of the Brauer tree.

Proof. If it were, then by the structure of projective modules in a Brauer tree algebra, the heart $\operatorname{Rad}\left(P_{C, W}\right) / \operatorname{Soc}\left(P_{C, W}\right)$ of the projective cover $P_{C, W}$ would be the direct sum of two uniserial modules, one of which having only $S_{C, W}^{k}$ as composition factors. Then there would be a non-trivial extension between $S_{C, W}^{k}$ and itself, contradicting 14.3, because $\operatorname{Ext}_{k \bar{N}_{G}(C)}(W, W)=0$ since $\bar{N}_{G}(C)$ has order prime to $p$.

Another proof is the following. If $T_{\text {Mod }}$ has an exceptional vertex, then it must remain exceptional in $T_{\text {Mack }}$ and so the free end of the twig $S_{C, W}^{k}$ (which is not in $T_{\text {Mod }}$ ) cannot be exceptional. So we can assume that $T_{\text {Mod }}$ has no exceptional vertex, in which case we want to prove that $T_{\text {Mack }}$ has no exceptional vertex. But this property is preserved by the stable equivalence between $G$ and $N_{G}(C)$. This follows from the arguments of the last section of [3], using the determinant of the Cartan matrix. Thus we can assume that $C$ is normal, but then the result is clear by 20.1 .

We now sum up the situation as follows.

(20.10) Theorem. Let $T_{\text {Mack }}$ be the Brauer tree of a block of Mackey functors in a situation where $G$ has a Sylow p-subgroup $C$ of order $p$ and we are working over a splitting $p$-modular system $(k, \mathscr{O}, K)$. The vertices of $T_{\text {Mack }}$ may be labelled with the simple Mackey functors over $K$, in such a manner that each simple decomposes to give the simple Mackey functors over $k$ which label the surrounding edges (each simple Mackey functor over $k$ appearing once). The edges and vertices of $T_{\text {Mack }}$ labelled by the simples indexed by the trivial subgroup form a subtree isomorphic to the corresponding Brauer tree $T_{\mathrm{Mod}}$ for $G$-modules. Each remaining edge is a twig fastened to $T_{\mathrm{Mod}}$. If $e$ denotes the number of edges of $T_{\mathrm{Mod}}$, then there are also $e$ edges outside $T_{\mathrm{Mod}}$. In particular the 
number of vertices of $T_{\text {Mack }}$ is $2 e+1$. The exceptional vertex is labelled with all of the simples $S_{1, U}^{K}$ for which the $K G$-modules $U$ label the exceptional vertex of $T_{\text {Mod }}$.

Proof. Let us first compute the number of edges. It is known from the theory of cyclic defect groups that the number $e$ of simple $k G$-modules in the block $b$ is equal to the number of simple $k N_{G}(C)$-modules $W$ in the block $b^{\prime}$, where $b^{\prime}$ is the Brauer correspondent of $b$. Each such module $W$ gives rise to a simple Mackey functor $S_{C, W}^{k}$ lying in the block $\hat{b}$ of Mackey functors corresponding to $b$. These simples $S_{C, W}^{k}$ are precisely the edges of $T_{\text {Mack }}$ which do not lie in $T_{\text {Mod }}$. So there are $e$ such edges, as required.

We have already labelled the vertices in the subtree $T_{\text {Mod }}$, and for the remaining vertices we label the free end of the twig $S_{C, W}^{k}$ with its lifting to characteristic 0 , namely $S_{C, W}^{K}$. It only remains to show that each vertex decomposes to give the surrounding edges. This is clear for $S_{C, W}^{K}$ because $d\left(S_{C, W}^{K}\right)=S_{C, W}^{k}$ by 20.5 and $S_{C, W}^{K}$ is the free end of the twig $S_{C, W}^{k}$. Consider now the decomposition of $S_{1, U}^{K}$. By 20.5 the terms in $T_{\mathrm{Mod}}$ are exactly the edges $V_{i}$ in $T_{\text {Mod }}$ surrounding $U$. So we only have to deal with the case of a simple $S_{C, W}^{k}$ appearing in $d\left(S_{1, U}^{K}\right)$.

We want to prove that the decomposition number $d\left(S_{1, U}^{K}, S_{C, W}^{k}\right)$ is nonzero if and only if $S_{C, W}^{k}$ is fastened to the vertex $S_{1, U}^{K}$. If the number of all edges $V_{i}$ emanating from $U$ is at least 2 , then since we have a tree the result is clear by 20.7. But if there is a single edge $V_{1}$ in $T_{\text {Mod }}$ emanating from $U$, it could be (using again 20.7) that $d\left(S_{1, U}^{K}, S_{C, W}^{k}\right)$ is non-zero and that $S_{C, W}^{k}$ is fastened to the other end $U_{2}$ of $V_{1}$. We now wish to eliminate this possibility. We write $U_{1}=U$ and assume that $S_{C, W}^{k}$ appears in $d\left(S_{1, U_{1}}^{K}\right)$ and that $S_{C, W}^{k}$ is fastened to $U_{2}$.

First assume that there is another edge $V_{2}$ in $T_{\text {Mod }}$ emanating from $U_{2}$, with end $U_{2}$ and $U_{3}$. Then $T_{\text {Mack }}$ has a subtree of the following shape:

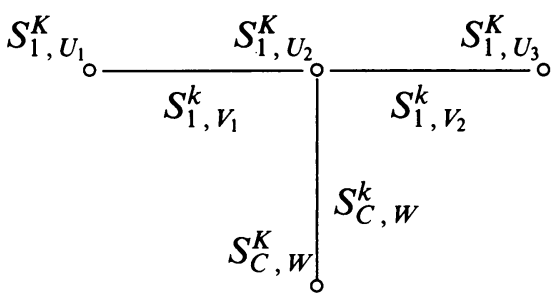

Let $m_{i}$ be the multiplicity of the vertex $U_{i}$ in $T_{\text {Mod }}$ and let $U_{i}^{1}, \ldots, U_{i}^{m_{i}}$ be the simples in characteristic zero labelling the vertex $U_{i}$. Of course at most one of the vertices has multiplicity greater than 1 , so at least two of $m_{1}, m_{2}, m_{3}$ are equal to 1, but we do not know which ones. We are interested in the occurrence of $S_{C, W}^{k}$ in the decomposition of $S_{1, U_{i}^{j}}^{K}$ so we write the decomposition numbers

$$
\begin{array}{ll}
d\left(S_{1, U_{1}^{j}}^{K}, S_{C, W}^{k}\right)=a_{j}, & 1 \leq j \leq m_{1}, \\
d\left(S_{1, U_{2}^{j}}^{K}, S_{C, W}^{k}\right)=b_{j}, & 1 \leq j \leq m_{2}, \\
d\left(S_{1, U_{3}^{K}}^{K}, S_{C, W}^{k}\right)=c_{j}, & 1 \leq j \leq m_{3} .
\end{array}
$$


By assumption at least one $a_{j}$ is non-zero. Since $V_{1}$ appears only in the decomposition of $U_{1}^{j}$ and $U_{2}^{j}$, we obtain the Cartan integer

$$
\begin{aligned}
c\left(S_{1, V_{1}}^{k}, S_{C, W}^{k}\right)= & \sum_{j=1}^{m_{1}} d\left(S_{1, U_{1}^{j}}^{K}, S_{1, V_{1}}^{k}\right) d\left(S_{1, U_{1}^{j}}^{K}, S_{C, W}^{k}\right) \\
& \quad+\sum_{j=1}^{m_{2}} d\left(S_{1, U_{2}^{j}}^{K}, S_{1, V_{1}}^{k}\right) d\left(S_{1, U_{2}^{j}}^{K}, S_{C, W}^{k}\right) \\
= & \sum_{j=1}^{m_{1}} a_{j}+\sum_{j=1}^{m_{2}} b_{j}
\end{aligned}
$$

and similarly $c\left(S_{1, v_{2}}^{k}, S_{C, W}^{k}\right)=\sum_{j} b_{j}+\sum_{j} c_{j}$. Also we have

$$
\begin{aligned}
c\left(S_{C, W}^{k}, S_{C, W}^{k}\right) & =d\left(S_{C, W}^{K}, S_{C, W}^{k}\right)+\sum_{U} d\left(S_{1, U}^{K}, S_{C, W}^{k}\right)^{2} \\
& \geq 1+\sum_{j} a_{j}^{2}+\sum_{j} b_{j}^{2}+\sum_{j} c_{j}^{2} .
\end{aligned}
$$

On the other hand from the structure of Brauer tree algebras, we have $c\left(S_{1, V_{1}}^{k}, S_{C, W}^{k}\right)=m_{2}, \quad c\left(S_{1, V_{2}}^{k}, S_{C, W}^{k}\right)=m_{2}, \quad c\left(S_{C, W}^{k}, S_{C, W}^{k}\right)=m_{2}+1$, using for the last equality the fact that the vertex $S_{C, W}^{K}$ is not exceptional (by 20.9). Putting together these values of Cartan integers, we first obtain

$$
\sum_{j=1}^{m_{3}} c_{j}=\sum_{j=1}^{m_{1}} a_{j}
$$

and it follows that at least one $c_{j}$ is non-zero. Thus we have

$$
m_{2}=\sum a_{j}+\sum b_{j} \leq \sum a_{j}^{2}+\sum b_{j}^{2}<\sum a_{j}^{2}+\sum b_{j}^{2}+\sum c_{j}^{2} \leq m_{2},
$$

a contradiction.

Now we assume that there is no other edge in $T_{\text {Mod }}$ emanating from $U_{2}$, so that both $U_{1}$ and $U_{2}$ are ends of the tree $T_{\text {Mod }}$. This implies that $T_{\text {Mod }}$ just consists in the two vertices $U_{1}$ and $U_{2}$ joined by the edge $V_{1}=V$. By the first part of the proof, we know that $T_{\text {Mack }}$ has only one extra vertex, and the whole tree looks as follows:

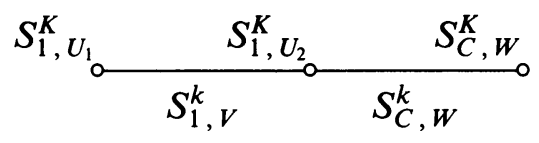

Recall that the hypothesis we are trying to eliminate is that $S_{C, W}^{k}$ appears in $d\left(S_{1, U_{1}}^{K}\right)$. We can proceed as follows.

Let $m_{i}$ be the multiplicity of $U_{i}$ and write $U_{i}^{1}, \ldots, U_{i}^{m_{i}}$ for the simples in characteristic zero labelling the vertex $U_{i}$. Of course at most one of $m_{1}, m_{2}$ is greater than 1 . We set

$$
a=d\left(S_{1, U_{1}^{j}}^{K}, S_{C, W}^{k}\right), \quad b=d\left(S_{1, U_{2}^{j}}^{K}, S_{C, W}^{k}\right) .
$$


Each of these numbers is independent of $j$ by 20.6. Our assumption is that $a \neq 0$. Then we immediately obtain the Cartan integers

$$
\begin{aligned}
c\left(S_{1, V}^{k}, S_{C, W}^{k}\right) & =m_{1} a+m_{2} b, \\
c\left(S_{C, W}^{k}, S_{C, W}^{k}\right) & =m_{1} a^{2}+m_{2} b^{2}+1
\end{aligned}
$$

which by the structure of the Brauer tree algebra are also equal to

$$
c\left(S_{1, V}^{k}, S_{C, W}^{k}\right)=m_{2}, \quad c\left(S_{C, W}^{k}, S_{C, W}^{k}\right)=m_{2}+1,
$$

using for the last equality the fact that the vertex $S_{C, W}^{K}$ is not exceptional (by 20.9). It follows that

$$
m_{1} a+m_{2} b=m_{1} a^{2}+m_{2} b^{2}=m_{2}
$$

which implies first that $a$ and $b$ are either 0 or 1 , and then that $b=0$ because $a \neq 0$. Thus $a=1$ and $m_{1}=m_{2}$. But there is at most one exceptional vertex, so $m_{1}=m_{2}=1$. Now we are in a situation where it is impossible to distinguish between the two vertices of $T_{\text {Mod }}$, so we can exchange them. Then the extra vertex $S_{C, W}^{K}$ is fastened precisely to the vertex of $T_{\text {Mod }}$ for which the corresponding decomposition number is non-zero, as required.

Now we know that $S_{C, W}^{k}$ is fastened to $S_{1, U}^{K}$ if and only if $d\left(S_{1, U}^{K}, S_{C, W}^{k}\right) \neq$ 0 . We compute the Cartan integers from the decomposition numbers in order to show that each such non-zero decomposition number has to be equal to 1 and that also it must be constantly equal to 1 for the exceptional vertex. Let $m$ be the multiplicity of the vertex $U$ and let $U^{1}, \ldots, U^{m}$ be the simple $K G$ modules labelling this vertex. We assume that $S_{C, W}^{k}$ is fastened to $S_{1, U}^{K}$ so that at least one of the decomposition numbers $a_{j}=d\left(S_{1, U^{j}}^{K}, S_{C, W}^{k}\right)$ is nonzero. If $V$ is some edge in $T_{\text {Mod }}$ with end vertex $U$, then the decomposition number $d\left(S_{1, U^{j}}^{K}, S_{1, V}^{k}\right)$ is equal to one and so we obtain the Cartan integer

$$
c\left(S_{1, V}^{k}, S_{C, W}^{k}\right)=\sum_{j=1}^{m} d\left(S_{1, U^{j}}^{K}, S_{1, V}^{k}\right) d\left(S_{1, U^{j}}^{K}, S_{C, W}^{k}\right)=\sum_{j=1}^{m} a_{j}
$$

which is also equal to $m$ by the structure of a Brauer tree algebra. Similarly

$$
c\left(S_{C, W}^{k}, S_{C, W}^{k}\right)=d\left(S_{C, W}^{K}, S_{C, W}^{k}\right)^{2}+\sum_{j=1}^{m} d\left(S_{1, U^{j}}^{K}, S_{C, W}^{k}\right)^{2}=1+\sum_{j=1}^{m} a_{j}^{2}
$$

and this is equal to $1+m$ by the structure of a Brauer tree algebra. It follows that

$$
m=\sum_{j=1}^{m} a_{j}=\sum_{j=1}^{m} a_{j}^{2}
$$

and this implies that $a_{j}=1$ for all $j$, as required. This argument works of course if $m=1$. The proof of Theorem 20.10 is now complete.

(20.11) Corollary. Let $U^{1}, \ldots, U^{m}$ be the simple $K G$-modules labelling the exceptional vertex of $T_{\text {Mod }}$. Then the $K\left[N_{G}(C) / C\right]$-modules $\left(U^{j}\right)^{C}$ are all isomorphic. 
Proof. We use the fact that all simple Mackey functors $S_{1, U^{j}}^{K}$ decompose in the same way by the theorem above. By 9.10 , we deduce that all $K\left[N_{G}(C) / C\right]$ modules $S_{1, U^{j}}^{K}(C)=\left(U^{j}\right)^{C}$ decompose in the same way. Since $N_{G}(C) / C$ has order prime to $p$, it follows that these modules are isomorphic.

\section{APPENDIX: TABLES}

We present as examples the matrices $\Psi$ introduced in Section 6 , and also the decomposition matrices and Cartan matrices of Mackey functors for some small groups. We always give only the information relating to the blocks in $\operatorname{Mack}_{k}(G, 1)$, which contain precisely the simple and projective Mackey functors indexed by $p$-groups. Thus, for instance, we only give the entries in $\Psi$ which are indexed by such simple Mackey functors, and this is sufficient to determine the composition factors of a Mackey functor in $\operatorname{Mack}_{k}(G, 1)$ by 9.10 . The Mackey functors in other blocks may be computed from this case using 10.1. In situations where the behaviour of Mackey functors is described by a Brauer tree we give just the tree, and not the decomposition or Cartan matrices, which are deducible from it.

We adopt an ad hoc notation for representations, mostly referring to them by their dimensions. If $\zeta$ is an $n$th root of unity, we denote by $\zeta$ a 1-dimensional representation in the group generated by $\zeta$. Generally $\omega$ will be a primitive cube root of unity.

$C_{p}$

The matrices $\Psi$ :

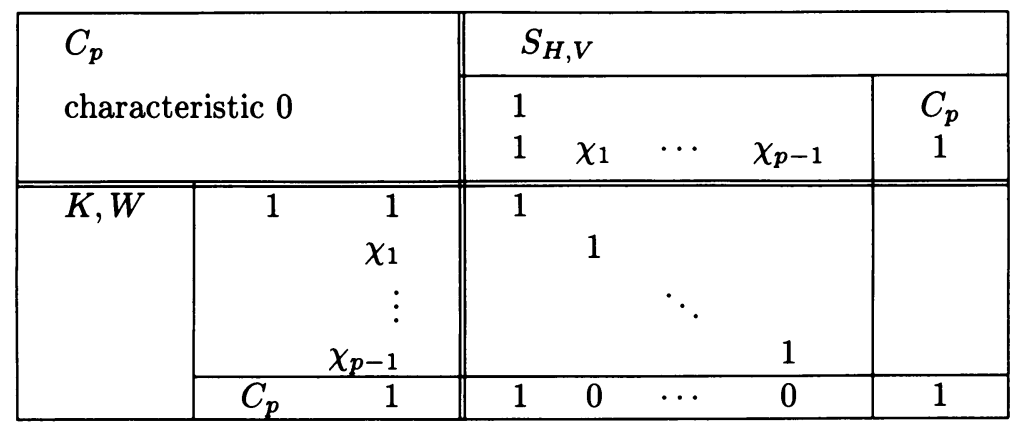

\begin{tabular}{|c|c|c|c|c|}
\hline \multirow{2}{*}{\multicolumn{2}{|c|}{$\begin{array}{l}C_{p} \\
\text { characteristic } p\end{array}$}} & & \multicolumn{2}{|c|}{$S_{H, V}$} \\
\hline & & & \multirow{2}{*}{$\begin{array}{l}1 \\
1 \\
1\end{array}$} & \multirow{2}{*}{$\begin{array}{c}C_{p} \\
1\end{array}$} \\
\hline \multirow[t]{2}{*}{$\bar{K}, W$} & $\overline{\overline{1}}$ & $\overline{1} \overline{1}$ & & \\
\hline & & 1 & & 1 \\
\hline
\end{tabular}

Brauer tree:

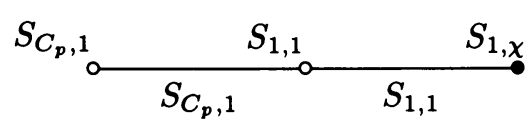

$\chi$ ranges through the $p-1$ non-identity characters $\chi_{1}, \ldots, \chi_{p-1}$. 
$S_{3}$

The matrices $\Psi$ :

\begin{tabular}{|c|c|c|c|c|c|c|c|c|c|}
\hline \multirow{2}{*}{\multicolumn{3}{|c|}{$\begin{array}{l}S_{3} \\
\text { characteristic } 0\end{array}$}} & \multicolumn{7}{|c|}{$S_{H, V}$} \\
\hline & & & $\begin{array}{l}1 \\
1 \\
1\end{array}$ & \multicolumn{2}{|c|}{$\begin{array}{ll}-1 & 2\end{array}$} & $\begin{array}{c}C_{2} \\
1\end{array}$ & $\begin{array}{c}C_{3} \\
1\end{array}$ & -1 & $\begin{array}{c}S_{3} \\
1\end{array}$ \\
\hline \multirow[t]{4}{*}{$\bar{K}, W$} & & $\begin{array}{r}1 \\
-1 \\
2\end{array}$ & & 1 & 1 & & & & \\
\hline & $C_{2}$ & 1 & 1 & & 1 & 1 & & & \\
\hline & $C_{3}$ & $\begin{array}{r}1 \\
-1\end{array}$ & 1 & 1 & & & 1 & 1 & \\
\hline & $S_{3}$ & 1 & 1 & & & 1 & 1 & & 1 \\
\hline
\end{tabular}

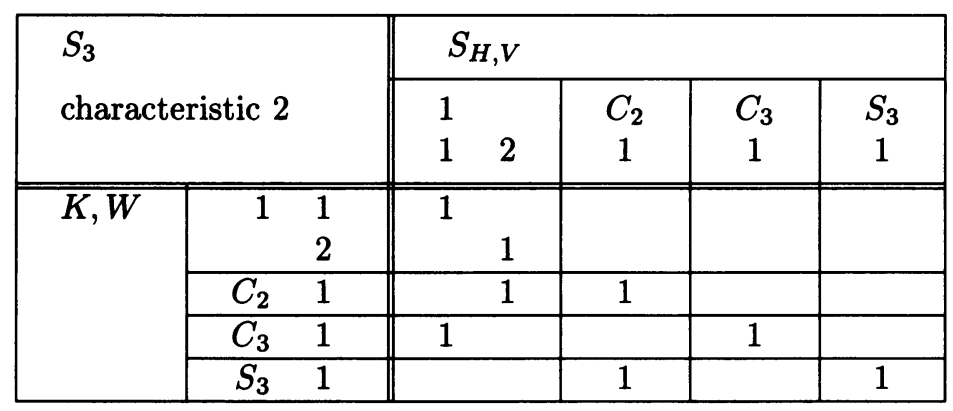

Brauer trees:

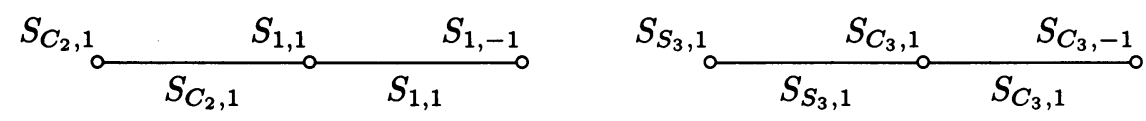

Simple projective: $S_{1,2}$ 
$S_{3}$

The matrix $\Psi$ :

\begin{tabular}{|c|c|c|c|c|c|c|c|c|}
\hline \multirow{2}{*}{\multicolumn{3}{|c|}{$\begin{array}{l}S_{3} \\
\text { characteristic } 3\end{array}$}} & \multicolumn{6}{|c|}{$S_{H, V}$} \\
\hline & & & \multirow{2}{*}{\multicolumn{2}{|c|}{$\begin{array}{cc}1 & \\
1 & -1 \\
1 & \\
& 1\end{array}$}} & \multirow{2}{*}{$\begin{array}{c}C_{2} \\
1\end{array}$} & \multirow{2}{*}{$\begin{array}{r}C \\
1\end{array}$} & \multirow{2}{*}{-1} & \multirow{2}{*}{$\begin{array}{c}S_{3} \\
1\end{array}$} \\
\hline \multirow[t]{4}{*}{$K, W$} & $\overline{1}$ & $\begin{array}{r}1 \\
-1\end{array}$ & & & & & & \\
\hline & & 1 & 1 & & 1 & & & \\
\hline & & $\begin{array}{r}1 \\
-1\end{array}$ & & & & 1 & 1 & \\
\hline & $S_{3}$ & 1 & & & 1 & 1 & & 1 \\
\hline
\end{tabular}

Brauer tree:

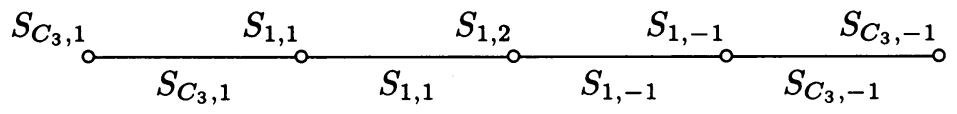

Simple projectives: $S_{C_{2}, 1}, S_{S_{3}, 1}$ 
$A_{5}$

The matrices $\Psi$ :

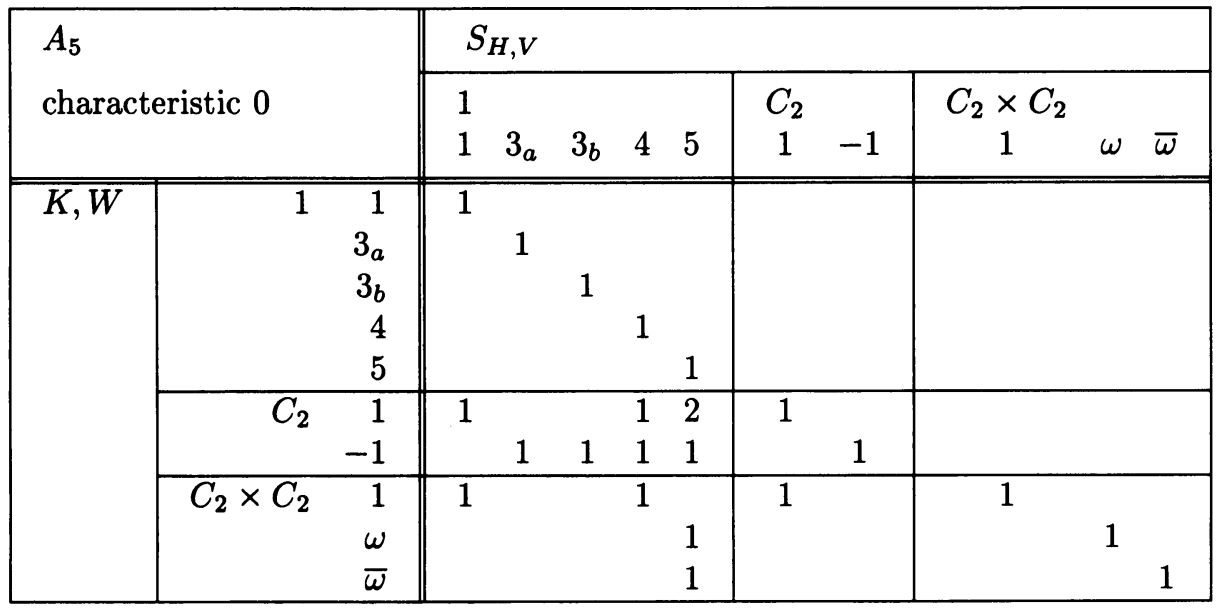

\begin{tabular}{|c|c|c|c|c|c|c|c|c|c|c|}
\hline \multirow{2}{*}{\multicolumn{2}{|c|}{$\begin{array}{l}A_{5} \\
\text { characteristic } 2\end{array}$}} & & \multicolumn{8}{|c|}{$S_{H, V}$} \\
\hline & & & \multicolumn{4}{|c|}{1} & $\begin{array}{c}C_{2} \\
1\end{array}$ & $\begin{array}{c}C_{2} \times C_{2} \\
1 \\
\end{array}$ & $\omega$ & \\
\hline 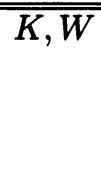 & 1 & $\begin{array}{r} \\
2 \\
2 a \\
2 b \\
4\end{array}$ & $\overline{\overline{1}}$ & 1 & 1 & 1 & & & & \\
\hline & $\overline{C_{2}}$ & 1 & & 1 & 1 & 2 & 1 & & & \\
\hline & $C_{2} \times C_{2}$ & $\begin{array}{l}1 \\
\omega \\
\bar{\omega}\end{array}$ & & & & 1 & & 1 & 1 & 1 \\
\hline
\end{tabular}


$A_{5}$

Decomposition matrix:

\begin{tabular}{|c|c|c|c|c|c|c|}
\hline \multirow{2}{*}{\multicolumn{2}{|c|}{$\begin{array}{l}A_{5} \\
\text { characteristic } 2\end{array}$}} & & \multicolumn{4}{|c|}{$S_{H, V}$} \\
\hline & & & 1 & & $?$ & \\
\hline$S_{K, W}$ & 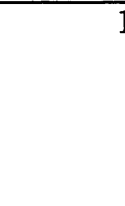 & $\begin{array}{r}1 \\
3 \\
3 \\
3 \\
4 \\
4 \\
5\end{array}$ & $\begin{array}{l}1 \\
1 \\
1\end{array}$ & 1 & 1 & 1 \\
\hline & $\begin{array}{l}C \\
C \\
C\end{array}$ & $\begin{array}{r}1 \\
-1 \\
\end{array}$ & & & & \\
\hline & $C_{2} \times C_{2}$ & $\begin{array}{c}1 \\
\omega \\
\bar{\omega}\end{array}$ & & & & 1 \\
\hline
\end{tabular}

Cartan matrix:

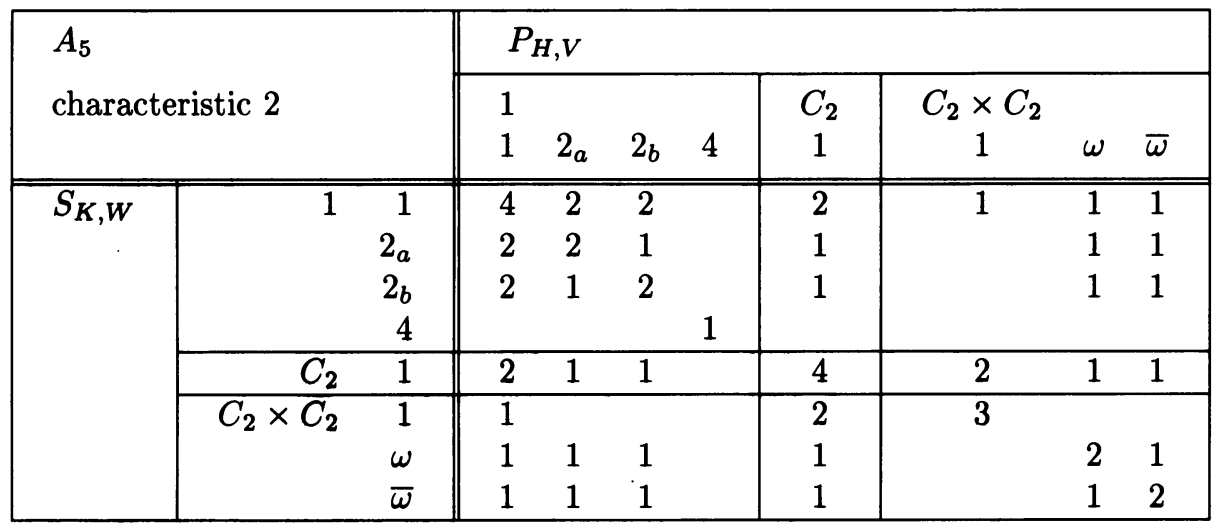


$G L(3,2)$

The matrices $\Psi$ :

\begin{tabular}{|c|c|c|c|c|c|c|c|c|c|}
\hline \multirow{2}{*}{\multicolumn{2}{|c|}{$\begin{array}{l}G L(3,2) \\
\text { characteristic } 0\end{array}$}} & \multicolumn{8}{|l|}{$S_{H, V}$} \\
\hline & & \multirow{2}{*}{ 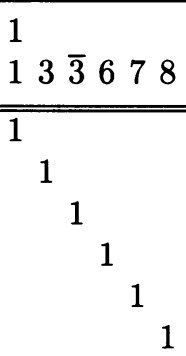 } & \multicolumn{2}{|c|}{$\begin{array}{ccccc}C_{2} & & & \\
1 & 1_{a} & 1_{b} & 1_{c}\end{array}$} & \multirow{2}{*}{\multicolumn{2}{|c|}{$\begin{array}{c}C_{4} \\
1-1\end{array}$}} & \multirow[t]{2}{*}{$\begin{array}{llll}V_{1} & & \\
1 & -1 & 2\end{array}$} & \multirow[t]{2}{*}{$\begin{array}{lll}V_{2} & \\
1 & -12\end{array}$} & \multirow[t]{2}{*}{$\begin{array}{c}D_{8} \\
1\end{array}$} \\
\hline \multirow[t]{6}{*}{$\bar{~} K, W$} & $\begin{array}{ll}1 & 1 \\
& 3 \\
& \\
& \\
& \\
& \\
& \\
& 7 \\
& \\
\end{array}$ & & & & & & & & \\
\hline & $\begin{array}{lr}C_{2} & 1 \\
& 1_{a} \\
& 1_{b} \\
& 1_{c}\end{array}$ & $\begin{array}{llllll}1 & & & & & 1 \\
& 1 & 1 & & 1 & 1 \\
& & & 1 & 1 & 1 \\
& & & 1 & 1 & 1\end{array}$ & 1 & 1 & & & & & \\
\hline & $\begin{array}{lr}C_{4} & 1 \\
& -1 \\
\end{array}$ & 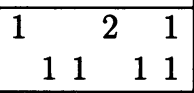 & 1 & 1 & & 1 & & & \\
\hline & $\begin{array}{rr}V_{1} & 1 \\
& -1 \\
& 2\end{array}$ & $\begin{array}{lllll}1 & & 1 & & \\
& & & 1 & \\
& & 1 & 1\end{array}$ & 1 & & & & $\begin{array}{lll}1 & & \\
& & \\
& & \\
& & 1\end{array}$ & & \\
\hline & $\begin{array}{lr}V_{2} & 1 \\
& -1 \\
& 2\end{array}$ & $\begin{array}{lllll}1 & & 1 & & \\
& & & 1 & \\
& & & & 1\end{array}$ & 1 & & & & & $\begin{array}{|lll|}1 & & \\
& 1 & \\
& & 1\end{array}$ & \\
\hline & $\begin{array}{ll}D_{8} & 1\end{array}$ & $\begin{array}{|ll|}2 & 1 \\
\end{array}$ & 3 & 1 & & 1 & 1 & 1 & 1 \\
\hline
\end{tabular}

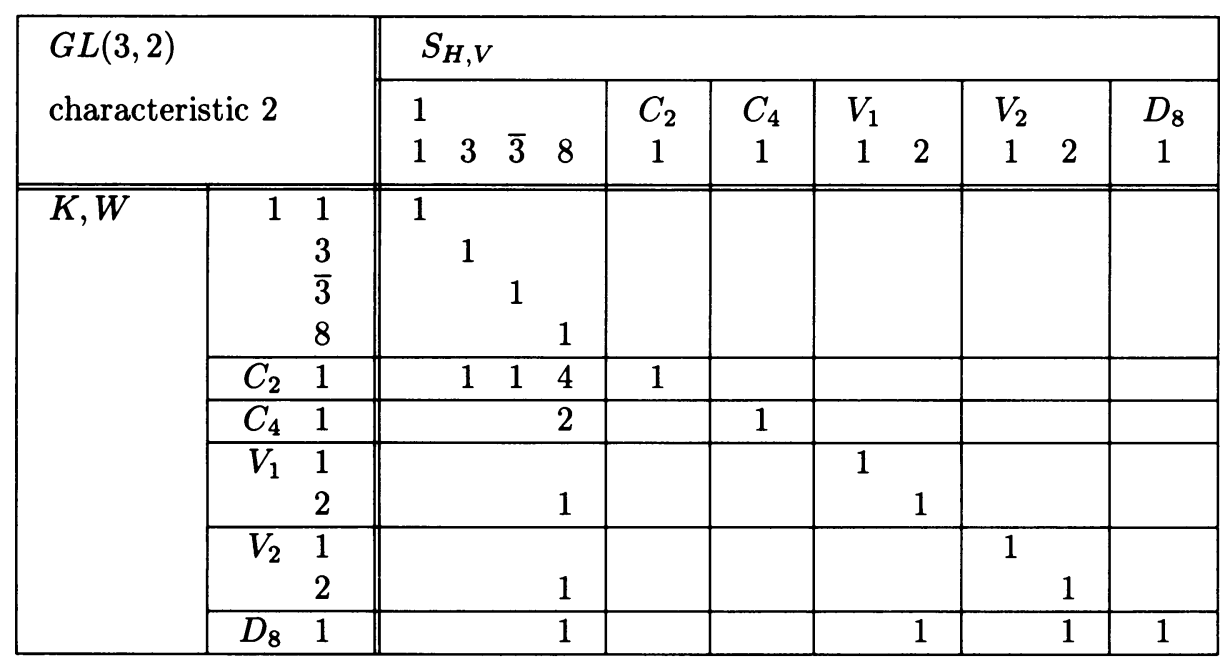


$G L(3,2)$

Decomposition matrix:

\begin{tabular}{|c|c|c|c|c|c|c|c|c|c|c|c|c|}
\hline \multirow{2}{*}{\multicolumn{3}{|c|}{$\begin{array}{l}G L(3,2) \\
\text { characteristic } 2\end{array}$}} & \multicolumn{10}{|c|}{$S_{H, V}$} \\
\hline & & & \multicolumn{3}{|c|}{$\begin{array}{llll}1 & & & \\
1 & 3 & \overline{3} & 8\end{array}$} & $\begin{array}{c}C_{2} \\
1 \\
1\end{array}$ & $\begin{array}{c}C_{4} \\
1 \\
\end{array}$ & \multicolumn{2}{|c|}{$\begin{array}{cc}V_{1} & \\
1 & 2\end{array}$} & \multicolumn{2}{|c|}{$\begin{array}{cc}V_{2} & \\
1 & 2\end{array}$} & $\begin{array}{c}D_{8} \\
1 \\
1\end{array}$ \\
\hline \multirow[t]{6}{*}{$S_{K, W}$} & & $\begin{array}{l}1 \\
3 \\
\overline{3} \\
6 \\
7 \\
8\end{array}$ & & $\begin{array}{l}1 \\
1 \\
1\end{array}$ & 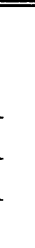 & $\begin{array}{l}2 \\
1\end{array}$ & $\begin{array}{l}1 \\
1 \\
1 \\
2 \\
1\end{array}$ & $\begin{array}{l}1 \\
1\end{array}$ & 1 & $\begin{array}{l}1 \\
1\end{array}$ & 1 & \\
\hline & & $\begin{array}{c}1 \\
1_{a} \\
1_{b} \\
1_{c}\end{array}$ & & & & $\begin{array}{l}1 \\
1 \\
1 \\
1 \\
\end{array}$ & $\begin{array}{l}1 \\
1\end{array}$ & & $\begin{array}{l}1 \\
1\end{array}$ & $\begin{array}{l}1 \\
1 \\
\end{array}$ & 1 & 1 \\
\hline & & $\begin{array}{r}1 \\
-1 \\
\end{array}$ & & & & & $\begin{array}{l}1 \\
1\end{array}$ & & & & & 1 \\
\hline & & $\begin{array}{r}1 \\
-1 \\
2\end{array}$ & & & & & & $\begin{array}{l}1 \\
1\end{array}$ & 1 & & & 1 \\
\hline & & $\begin{array}{r}1 \\
-1 \\
2\end{array}$ & & & & & & & & $\begin{array}{l}1 \\
1\end{array}$ & 1 & 1 \\
\hline & $\bar{D}$ & 1 & & & & & & & & & & 1 \\
\hline
\end{tabular}

Cartan matrix:

\begin{tabular}{|c|c|c|c|c|c|c|c|c|c|c|c|c|}
\hline \multirow{2}{*}{\multicolumn{2}{|c|}{$\begin{array}{l}G L(3,2) \\
\text { characteristic } 2\end{array}$}} & & \multicolumn{10}{|c|}{$P_{H, V}$} \\
\hline & & & & $\begin{array}{c}C_{2} \\
1\end{array}$ & $\begin{array}{c}C_{4} \\
1\end{array}$ & \multicolumn{2}{|c|}{$\begin{array}{cc}V_{1} & \\
1 & 2\end{array}$} & \multicolumn{2}{|c|}{$\begin{array}{cc}V_{2} & \\
1 & 2\end{array}$} & $\begin{array}{c}D_{8} \\
1\end{array}$ \\
\hline \multirow[t]{8}{*}{$S_{K, W}$} & & $\begin{array}{l} \\
3 \\
\overline{3} \\
8\end{array}$ & & 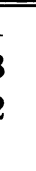 & $\begin{array}{l}1 \\
2 \\
3\end{array}$ & $\begin{array}{l}2 \\
3 \\
3\end{array}$ & $\begin{array}{l}2 \\
4 \\
4\end{array}$ & $\begin{array}{l}2 \\
2 \\
2\end{array}$ & $\begin{array}{l}1 \\
1\end{array}$ & $\begin{array}{l}2 \\
2 \\
2\end{array}$ & $\begin{array}{l}1 \\
1\end{array}$ & $\begin{array}{ll}1 \\
\end{array}$ \\
\hline & $C_{2}$ & 1 & 2 & 3 & 3 & 10 & 8 & 6 & 4 & 6 & 4 & 2 \\
\hline & $C_{4}$ & 1 & 2 & 4 & 4 & 8 & 12 & 5 & 3 & 5 & 3 & 3 \\
\hline & & 1 & & 2 & 2 & 6 & 5 & 7 & 3 & 4 & 2 & 3 \\
\hline & & 2 & & 1 & 1 & 4 & 3 & 3 & 4 & 2 & 2 & 1 \\
\hline & $V_{2}$ & & 2 & 2 & 2 & 6 & 5 & 4 & 2 & 7 & 3 & 3 \\
\hline & & 2 & & 1 & 1 & 4 & 3 & 2 & 2 & 3 & 4 & 1 \\
\hline & $D_{8}$ & 1 & 1 & & & 2 & 3 & 3 & 1 & 3 & 1 & 6 \\
\hline
\end{tabular}


$P S L(2,7)$

The matrices $\Psi$ :

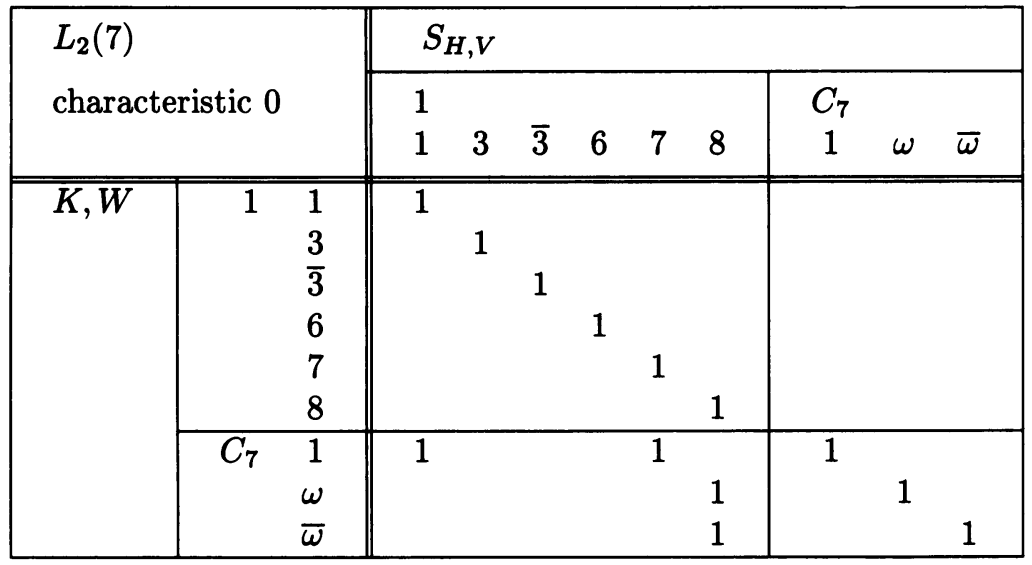

\begin{tabular}{|c|c|c|c|c|c|c|c|c|c|}
\hline \multirow{2}{*}{\multicolumn{3}{|c|}{$\begin{array}{l}L_{2}(7) \\
\text { characteristic } 7\end{array}$}} & \multicolumn{7}{|c|}{$S_{H, V}$} \\
\hline & & & $\begin{array}{l}1 \\
1 \\
1\end{array}$ & 3 & 5 & 7 & $\begin{array}{c}C_{7} \\
1\end{array}$ & $\omega$ & $\bar{\omega}$ \\
\hline $\bar{K}, W$ & $\overline{\overline{1}}$ & $\begin{array}{l}1 \\
3 \\
5 \\
7\end{array}$ & $\overline{\overline{1}}$ & 1 & 1 & 1 & & & \\
\hline & & $\begin{array}{c}1 \\
\omega \\
\bar{\omega}\end{array}$ & & & & 1 & 1 & 1 & 1 \\
\hline
\end{tabular}

Brauer tree:

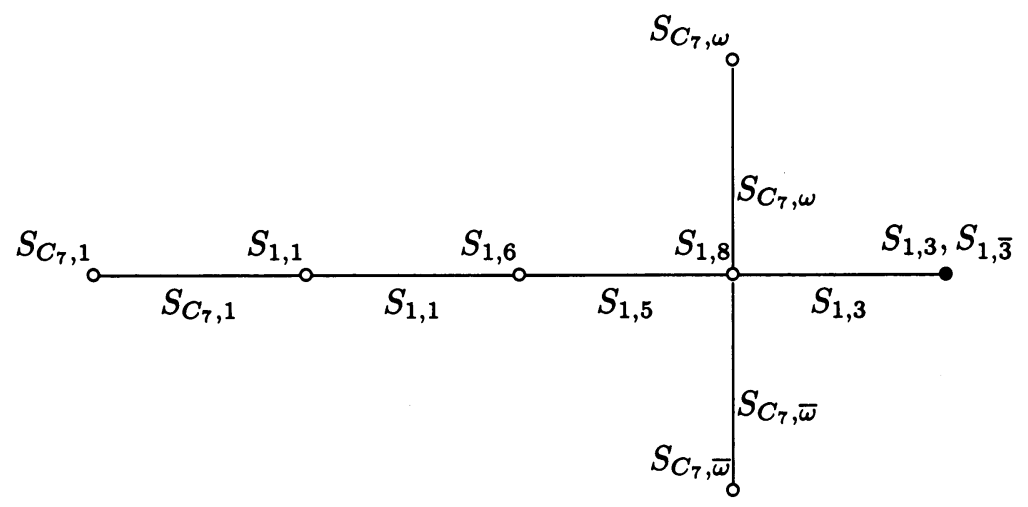

Simple projective: $S_{1,7}$ 


\section{REFERENCES}

1. J.L. Alperin, Diagrams for modules, J. Pure Appl. Algebra 16 (1980), 111-119.

2. Weights for finite groups, The Arcata Conference on Representations of Finite Groups (P. Fong, ed.), Proc. Sympos. Pure Math., vol. 47, Amer. Math. Soc., Providence, RI, 1987, pp. 369-379.

3. _ Local representation theory, Cambridge University Press, 1986.

4. J.L. Alperin and M. Broué, Local methods in block theory, Ann. of Math. 110 (1979), 143-157.

5. M. Broué, On Scott modules and p-permutation modules: an approach through the Brauer morphism, Proc. Amer. Math. Soc. 93 (1985), 401-408.

6. C.W. Curtis and I. Reiner, Methods of representation theory II, Wiley, 1987.

7. T. tom Dieck, Transformation groups, De Gruyter, Berlin-New York, 1987.

8. L. Dornhoff, Group representation theory, Marcel Dekker, New York, 1972.

9. A.W.M. Dress, Contributions to the theory of induced representations, Algebraic K-Theory II (H. Bass, ed.), Lecture Notes in Math., vol. 342, Springer-Verlag, 1973, pp. 183-240.

10. P. Gabriel and Ch. Riedtmann, Group representations without groups, Comment. Math. Helv. 54 (1979), 240-287.

11. J.A. Green, Axiomatic representation theory for finite groups, J. Pure Appl. Algebra 1 (1971), 41-77.

12. D.G. Higman, Indecomposable representations at characteristic p, Duke Math. J. 21 (1954), 369-376.

13. P.J. Hilton and U. Stammbach, A course in homological algebra, Graduate Texts in Math. 4, Springer-Verlag, 1970.

14. P. Landrock, Finite group algebras and their modules, LMS Lecture Notes 84, Cambridge University Press, 1983.

15. H. Lindner, A remark on Mackey functors, Manuscripta Math. 18 (1976), 273-278.

16. R. Oliver, Whitehead groups of finite groups, LMS Lecture Notes 132, Cambridge University Press, 1988.

17. H. Sasaki, Green correspondence and transfer theorems of Wielandt type for G-functors, J. Algebra 79 (1982), 98-120.

18. D. Tambara, Homological properties of the endomorphism ring of certain permutation modules, Osaka J. Math. 26 (1989), 807-828.

19. J. Thévenaz, Some remarks on G-functors and the Brauer morphism, J. Reine Angew. Math. 384 (1988), 24-56.

20. J. Thévenaz and P.J. Webb, Simple Mackey functors, Proc. of 2nd International Group Theory Conference, Bressanone (1989), Supplemento ai Rendiconti del Circolo Matematico di Palermo 23, 1990, pp. 299-319.

21. P.J. Webb, A split exact sequence of Mackey functors, Comment. Math. Helv. 66 (1991), 34-69.

22. A. Wiedemann, Elementary construction of the quiver of the Mackey algebra for groups with cyclic normal p-Sylow subgroup, J. Algebra 150 (1992), 296-307.

23. T. Yoshida, Idempotents of Burnside rings and Dress induction theorem, J. Algebra 80 (1983), 90-105.

24. $\_$, On G-functors II: Hecke operators and G-functors, J. Math. Soc. Japan 35 (1983), 179-190.

25. _ Idempotents and transfer theorems of Burnside rings, character rings and span rings, Algebraic and Topological Theories (to the memory of T. Miyata) (1985), 589-615.

Institut de Mathematiques, Universite de Lausanne, CH-1015 Lausanne, Switzerland E-mail address: jacques.thevenaz@ima.unil.ch

School of Mathematics, University of Minnesota, Minneapolis Minnesota 55455

E-mail address: webb@math.umn.edu 